\title{
VOLUME AVERAGING OF MULTIPHASE FLOWS WITH HYDRATE FORMATION IN SUBSEA PIPELINES
}

BY

(C) Reza Zeinali Torbati

\author{
A Thesis submitted to the \\ School of Graduate Studies \\ In partial fulfillment of the requirements for the degree of \\ Master of Engineering \\ Faculty of Engineering and Applied Science \\ Memorial University of Newfoundland
}

December 2015

St. John's

Newfoundland, Canada 


\begin{abstract}
In oil and gas pipeline operations, the gas, oil, and water phases simultaneously move through pipe systems. The mixture cools as it flows through subsea pipelines, and forms a hydrate formation region, where the hydrate crystals start to grow and may eventually block the pipeline. The potential of pipe blockage due to hydrate formation is one of the most significant flow-assurance problems in deep-water subsea operations. Due to the catastrophic safety and economic implications of hydrate blockage, it is important to accurately predict the simultaneous flow of gas, water, and hydrate particles in flowlines. Currently, there are few or no studies that account for the simultaneous effects of hydrate growth and heat transfer on flow characteristics within pipelines.
\end{abstract}

This thesis presents new and more accurate predictive models of multiphase flows in undersea pipelines to describe the simultaneous flow of gas, water, and hydrate particles through a pipeline. A growth rate model for the hydrate phase is presented and then used in the development of a new three-phase model. The conservation equations of mass, momentum, and energy are formulated to describe the physical phenomena of momentum and heat transfer between the fluid and the wall. The governing equations are solved based on an analytical-numerical approach using a Newton-Raphson method for the nonlinear equations. An algorithm was developed in Matlab software to solve the equations from the inlet to the outlet of the pipeline. The developed models are validated against a single-phase model with mixture properties, and the results of comparative studies show close agreement.

The new model predicts the volume fraction and velocity of each phase, as well as the mixture pressure and temperature profiles along the length of the pipeline. The results from the hydrate growth model reveal the growth rate and location where the initial hydrates start to form. Finally, to assess the impact of certain parameters on the flow characteristics, parametric studies have been conducted. The results show the effect of a variation in the pipe 
diameter, mass flow rate, inlet pressure, and inlet temperature on the flow characteristics and hydrate growth rates.

Key Words: Hydrate particles, multiphase flow, pipeline, natural gas 


\section{ACKNOWLEDGEMENTS}

I would like to express my sincere gratitude to my supervisor, Prof. Greg Naterer, and his research engineer, Dr. Adedoyin Odukoya, for their assistance and support throughout the course of my MEng Program, without which the completion of this work would not have happened. I would also like to thank the School of Graduate Studies at Memorial University of Newfoundland, RDC, and NSERC, for their financial support during my two-year program.

I am highly indebted to my lecturers during both my undergraduate and graduate studies for their exemplary tutelage and influence. My gratitude also extends to my friend, Dr. Hashem Nekouie, for providing me with deep knowledge and expertise in programming software.

Last, but not least, I owe my deepest gratitude to my wife and my parents for their endless support in my studies. Special thanks goes to my father who inspired me the most and made me who I am today. 


\section{TABLE OF CONTENTS}

Abstract.

Acknowledgments

Table of contents.

List of tables.

List of figures.

Nomenclature.

1. Introduction.

1.1 Multiphase systems.

1.2 Hydrate formation.

1.3 Objectives and outline of thesis.

2. Literature review

2.1 Multiphase flow models

2.2 Hydrate formation and growth models

3. Problem formulation.

Continuity equation........................................... 31

3.2 Momentum equation....................................... 32

$3.3 \quad$ Energy equation............................................. 39

$3.4 \quad$ Hydrate formation and growth................................. 41

4. Solutions of conservation equations................................. 45 


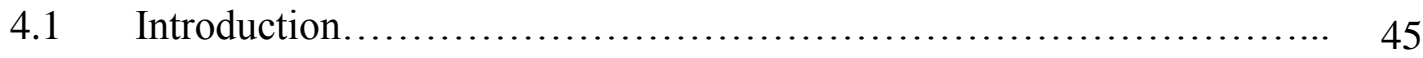

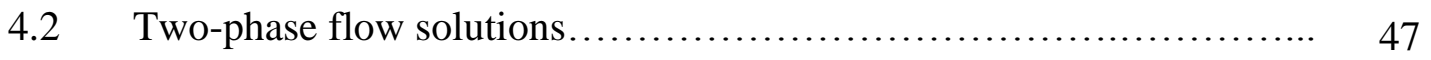

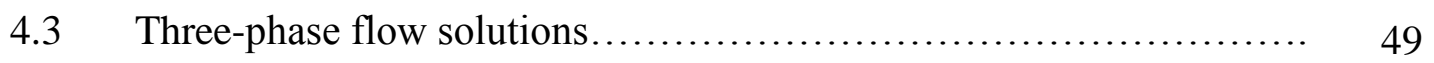

5. Results and discussion........................................... 53

$5.1 \quad$ Parameters and properties.................................. 53

$5.2 \quad$ Two-phase models........................................ 54

$5.3 \quad$ Three-phase model $\ldots \ldots \ldots \ldots \ldots \ldots \ldots \ldots \ldots \ldots \ldots \ldots \ldots \ldots \ldots \ldots \ldots, 62$

$5.4 \quad$ Parametric studies............................................. 65

6. Conclusions and recommendations for future research................... 77

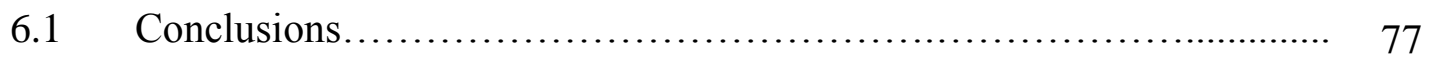

6.2 Recommendations for future research.......................... 78

References......................................................... 79 


\section{LIST OF TABLES}

Table 1. Problem parameters and properties

\section{LIST OF FIGURES}

Figure 1. Schematic relationship between pressure drop and flow rate in multiphase flow systems

Figure 2. Flow regimes for a two-phase steady-state flow in a horizontal pipe based on gas (X axis) and liquid (Y axis) superficial velocities (Bratland, 2010)

Figure 3. Different types of horizontal flows and flow regimes for gas/liquid flow in a horizontal pipe (Brennen, 2005)

Figure 4. Flow regimes for slurry flow (solid particles of sand dispersed in water) in a horizontal pipe

Figure 5. Pressure, temperature, and hydrate equilibrium line for different concentrations of methanol (Sloan, 2011)

Figure 6. Control volume representing three phases (gas, liquid, and solid)

Figure 7. A schematic of pressure force acting on the control volume in a pipe flow

Figure 8. Gas/water stratified flow

Figure 9. Friction force on the control volume

Figure 10. Cross-section of pipe for a stratified flow pattern

Figure 11. Distributions of steady-state velocity along the pipeline for stratified and annular flow models for different outlet pressures

Figure 12. Changes in liquid volume fraction along the pipeline for stratified and annular flow models for different outlet pressures

Figure 13. Changes in gas volume fraction along the pipeline for stratified and annular flow models for different outlet pressures

Figure 14. Gas density distribution along the pipeline for different outlet pressures 
Figure 15. Pressure profiles along the pipeline

Figure 16. Temperature profiles for different outlet pressures

Figure 17. Validation of the pressure profile for two-phase annular model against a singlephase model (Bar-Meir, 2013) with mixture properties

Figure 18. Validation of temperature profile for two-phase annular model against a singlephase model (Bergman et al., 2011) with mixture properties

Figure 19. Distributions of steady-state velocity along the pipeline for gas/hydrate annular flow model for different outlet pressures

Figure 20. Volume fraction profiles along the pipeline for gas/hydrate flow model for different outlet pressures

Figure 21. Temperature distribution along the pipeline for gas/hydrate flow model for different outlet pressures

Figure 22. Volume fraction of the phases along the pipeline length

Figure 23. Velocity of the phases along the pipeline length

Figure 24. Pressure distribution for the fluid mixture

Figure 25. Temperature distribution for the fluid mixture

Figure 26. Volume fraction distribution of the gas and hydrate along the pipeline for different pipe diameters

Figure 27. Volume fraction distribution of the liquid and hydrate along the pipeline for different pipe diameters

Figure 28. Velocity distribution of the gas and hydrate along the pipeline for different pipe diameters

Figure 29. Velocity distribution of the liquid and hydrate along the pipeline for different pipe diameters 
Figure 30. Temperature distribution of the mixture fluid along the pipeline for different pipe diameters

Figure 31 . Volume fraction distribution of the gas and hydrate along the pipeline for different mass flow rates

Figure 32. Volume fraction distribution of the liquid and hydrate along the pipeline for different mass flow rates

Figure 33. Temperature distribution of the mixture fluid along the pipeline for different mass flow

Figure 34. Volume fraction distribution of the gas and hydrate along the pipeline for different pressures

Figure 35. Volume fraction distribution of the liquid and hydrate along the pipeline for different pressures

Figure 36. Temperature distribution of the mixture fluid along the pipeline for different pressures

Figure 37. Volume fraction distribution of the gas and hydrate along the pipeline for different inlet temperatures

Figure 38. Volume fraction distribution of the liquid and hydrate along the pipeline for different inlet temperatures

Figure 39. Temperature distribution of the mixture fluid along the pipeline for different inlet temperatures 


\section{NOMENCLATURE}

Pipe cross-sectional area $\left[\mathrm{m}^{2}\right]$

$A_{S}$

Interfacial area between water and gas $\left[\mathrm{m}^{2}\right]$

$C_{P}$

Heat capacity $[\mathrm{J} / \mathrm{K}]$

$D$

Pipe diameter $[\mathrm{m}]$

$D_{h}$

Average diameter of the particles [m]

$e$

Pipe wall roughness height $[\mathrm{m}]$

$f$

Friction factor [-]

$f_{g h}$

Drag force coefficient [-]

$f_{g l}$

Interfacial friction factor between gas and liquid [-]

F

Force $[\mathrm{N}]$

$F_{g l}$

Friction force between gas and liquid $[\mathrm{N}]$

$F r_{l}$

Froude number for liquid phase [-]

$g$

Gravitational acceleration $\left[\mathrm{m} / \mathrm{s}^{2}\right]$

$h$

Heat transfer convection coefficient $\left[\mathrm{W} /\left(\mathrm{m}^{2} . \mathrm{K}\right)\right]$

$h_{i} \quad$ Inner fluid convection heat transfer coefficient $\left[\mathrm{W} /\left(\mathrm{m}^{2} . \mathrm{K}\right)\right]$

$h_{l} \quad$ Liquid height from the interface [m]

$h_{o} \quad$ Surrounding convection heat transfer coefficient $\left[\mathrm{W} /\left(\mathrm{m}^{2} . \mathrm{K}\right)\right]$

$H \quad$ Specific enthalpy $[\mathrm{J} / \mathrm{kg}]$

$k \quad$ Pipe conductivity [W/(m.K)] 


\begin{tabular}{|c|c|}
\hline$k_{1}$ & Intrinsic rate constant $[-]$ \\
\hline$k_{2}$ & Intrinsic rate constant $[\mathrm{K}]$ \\
\hline$k_{f}$ & Thermal conductivity of inner fluid $[\mathrm{W} /(\mathrm{m} . \mathrm{K})]$ \\
\hline$k_{h}$ & Thermal conductivity of hydrate particles $[\mathrm{W} /(\mathrm{m} . \mathrm{K})]$ \\
\hline$l$ & Pipe length $[\mathrm{m}]$ \\
\hline$\dot{m}_{g}$ & Gas phase mass flow rate $[\mathrm{kg} / \mathrm{s}]$ \\
\hline$\dot{m}_{l}$ & liquid phase mass flow rate $[\mathrm{kg} / \mathrm{s}]$ \\
\hline$\dot{m}_{h}$ & hydrate phase mass flow rate $[\mathrm{kg} / \mathrm{s}]$ \\
\hline$n_{H}$ & Hydration number [-] \\
\hline$p_{g l}$ & Gas/liquid interface $[\mathrm{m}]$ \\
\hline$p_{g w}$ & Wetted perimeter by gas phase $[\mathrm{m}]$ \\
\hline$p_{l w}$ & Wetted perimeter by liquid phase $[\mathrm{m}]$ \\
\hline$P$ & Pressure $[\mathrm{Pa}]$ \\
\hline$P r_{f}$ & Mixture Prandtl number [-] \\
\hline$\dot{Q}$ & Heat transfer per unit volume $\left[\mathrm{W} / \mathrm{m}^{3}\right]$ \\
\hline$r_{i}$ & Pipe inner radius [m] \\
\hline$r_{o}$ & Pipe outer radius $[\mathrm{m}]$ \\
\hline$R_{g h}$ & Hydrate particle drag force per unit volume $\left[\mathrm{N} / \mathrm{m}^{3}\right]$ \\
\hline$R e_{f}$ & Inner fluid Reynolds number [-] \\
\hline$R e_{g h}$ & Hydrate particle Reynolds number [-] \\
\hline
\end{tabular}




\begin{tabular}{|c|c|}
\hline$R e_{h}$ & Reynolds number of hydrate particles [-] \\
\hline$R e_{s l}$ & Reynolds number based on the superficial velocity [-] \\
\hline$S$ & Surface tension $[\mathrm{N} / \mathrm{m}]$ \\
\hline$t$ & Time $[\mathrm{s}]$ \\
\hline$T$ & Average fluid temperature $[\mathrm{K}]$ \\
\hline$T_{e q}$ & Hydrate equilibrium temperature $[\mathrm{K}]$ \\
\hline$T_{i}$ & Average inner fluid temperature $[\mathrm{K}]$ \\
\hline$T_{o}$ & Surrounding temperature $[\mathrm{K}]$ \\
\hline$U$ & Overall heat transfer coefficient [-] \\
\hline$v$ & Velocity $[\mathrm{m} / \mathrm{s}]$ \\
\hline$v_{g}$ & Gas velocity $[\mathrm{m} / \mathrm{s}]$ \\
\hline$v_{h}$ & Hydrate velocity $[\mathrm{m} / \mathrm{s}]$ \\
\hline$v_{l}$ & Liquid velocity $[\mathrm{m} / \mathrm{s}]$ \\
\hline$x$ & Distance from pipe inlet $[\mathrm{m}]$ \\
\hline$\alpha$ & Volume fraction [-] \\
\hline$\alpha_{g}$ & Gas phase volume fraction [-] \\
\hline$\alpha_{h}$ & Hydrate volume fraction [-] \\
\hline$\alpha_{l}$ & Liquid volume fraction [-] \\
\hline$\beta$ & Wetted angle by the gas phase [radians] \\
\hline$\Gamma$ & Mass transfer rate $[\mathrm{kg} / \mathrm{s}]$ \\
\hline
\end{tabular}


Frictional pressure drop $[\mathrm{Pa}]$

$\Delta P_{m} \quad$ Momentum pressure drop [Pa]

$\Delta P_{S} \quad$ Static pressure drop $[\mathrm{Pa}]$

$\Delta P_{\text {tot }} \quad$ Total pressure drop $[\mathrm{Pa}]$

$\Delta T_{\text {sub }} \quad$ Subcooling temperature $[\mathrm{K}]$

$\eta \quad$ Joule-Thomson coefficient $[\mathrm{K} / \mathrm{Pa}]$

$\theta \quad$ Angle between pipe and horizon [radians]

$\mu_{f} \quad$ Dynamic viscosity of the gas phase at fluid temperature [Pa.s, N.s $/ \mathrm{m}^{2}$ ]

$\mu_{f s} \quad$ Dynamic viscosity of the gas phase at wall temperature [Pa.s, N.s $/ \mathrm{m}^{2}$ ]

$\mu_{g} \quad$ Dynamic viscosity of gas phase $\left[\mathrm{m}^{2} / \mathrm{s}\right]$

$\rho \quad$ Density $\left[\mathrm{kg} / \mathrm{m}^{3}\right]$

$\rho_{c} \quad$ Density of the continuous phase $\left[\mathrm{kg} / \mathrm{m}^{3}\right]$

$\rho_{g} \quad$ Density of the gas phase $\left[\mathrm{kg} / \mathrm{m}^{3}\right]$

$\rho_{h} \quad$ Density of the hydrate phase $\left[\mathrm{kg} / \mathrm{m}^{3}\right]$

$\rho_{l} \quad$ Density of the liquid phase $\left[\mathrm{kg} / \mathrm{m}^{3}\right]$ 


\section{INTRODUCTION}

Natural gas is one of the most widely used resources with several applications, including for power generation and transportation systems. In the oil and gas industry, it is crucial to ensure safe and cost-effective ways for offshore oil and gas production. Subsea pipelines are commonly used for transporting large quantities of oil and gas. Failure of these transportation systems can have catastrophic consequences both economically and environmentally.

In flow assurance, it is important to ensure that multiphase mixtures of gas, water and oil are safely transported from reservoir to storage facilities. Flow assurance is becoming increasingly important as hydrocarbons now come from remote reservoirs and deepwater fields, bringing the need to design long pipelines to transport fluids under extreme conditions. Subsea pipelines normally operate in deep water, where the harsh conditions and terrain features may cause unstable operating conditions (Danielson, 2012).

\subsection{Multiphase systems}

There are a large number of flows in nature that involve a mixture of different phases. The main phases in multiphase flow systems in this thesis are gas, liquid, and solid. The concept of phase in multiphase systems arises in a broader sense. Multiphase flow occurs in many industries, one of which is the petroleum industry. In the oil and gas industry, there is a growing need to better understand the individual phases of gas, water, and oil, as fields become more economically marginal. This thesis, therefore, focuses on multiphase flow in pipelines to address these problems.

Multiphase flow is found in many other applications such as food, pharmaceutical, nuclear energy for electrical power generation, transportation of solids, development of petrochemicals, and many other industries. Therefore, advances in better understanding of multiphase flows could lead to improvements in several industry sectors, including high 
power electronics, combustion, power systems, and transportation of petroleum products (Hanratty, 2003).

Multiphase flow occurs in most oil and gas wells, as well as in pipelines transporting the produced mixtures. Understanding multiphase flows is much more complex than singlephase flows, as the phases in a multiphase system are distributed non-uniformly. Due to the differences in densities, the phases tend to separate and create different flow patterns or flow regimes. There is a classification of the different physical distributions of the phases in a pipe. As a result of the difference in densities and viscosities, shear stresses at the pipe wall are different for each phase. Also, macroscopic properties of the flow can be affected significantly by small-scale phase interactions. Due to the change in pressure and temperature, as well as complex heat and mass transfer between the phases and wall, predicting the flow characteristics in oil and gas systems is complicated. All of these characteristics are governed by the conservation equations of mass, momentum, and energy.

Due to the complexity of phase interactions, the large size of pipelines, and harsh subsea environments, multiphase flow predictions require complex models. Offshore developments in deep water in harsh arctic climates have brought unique challenges in the production of oil and gas. Due to the harsh environments, the capital costs in these regions are high. A high level of accuracy is required for the design of the production systems. The high pressures and low temperatures in the system can cause serious flow-assurance problems such as erosion, corrosion, and blockage in the pipe. A blockage in the system may be due to total or partial plugging from deposition of paraffin and the formation of hydrates. To prevent or reduce such flow assurance problems, several chemical solutions have been developed. Depending on the flow regime of the fluid, these chemicals may have limited effectiveness when injected into the mixture. In the past few decades, researchers have tried to improve the available models to better predict the behavior of multiphase flow in different 
flow patterns. Unlike empirical correlations, which are only useful for some specific ranges of input variables, mechanistic models can be used for a wide range of input variables. In addition, they are more accurate, but more complicated, and more difficult to understand. In an empirical method, the flow rates, liquid holdup and pressure drop are measured, and the flow pattern is observed through the system. In the development of empirical correlations, the experiments are usually conducted at steady-state conditions using different diameters, pipe inclinations, and fluids. Then the data are used to develop empirical correlations to predict the flow patterns, liquid holdup, friction factor, and pressure gradient. In the development of a numerical model, computer software programs are also developed for the modelling and solution.

In order to have successful operation of production systems, engineers require the ability to predict flow behavior for a variety of flow rates in the system. The flow rate in the system changes frequently due to the addition of flows from new wells and fields into a pipeline, or due to the changes resulted from flow assurance problems and maintenance issues. In order to successfully predict these behaviors, often a commercial multiphase-flow simulator like OLGA is used. OLGA solves the conservation equations and is able to predict the flow patterns and transient behaviour of systems.

Prediction of the pressure drop in multiphase flows is more complicated than in single-phase flows. In single-phase flows, higher fluid flow rates lead to a higher pressure drop along the pipeline. For higher flow rates in multiphase flows, the pressure drop due to friction plays a key role in the pressure equation. It is different for lower flow rates, where the gravitational pressure drop is the dominant term among all the pressure drops (Fig. 1). Therefore, multiphase flows generally do not behave like single-phase flows and a higher pressure loss often occurs at lower flowrates as well. In fact, at lower flowrates, liquid accumulates and gradually builds up in the lowest points, then liquid flows up in the pipeline. 
When there is not enough gas to blow out accumulated liquid, the pressure is increased in the gas pocket, which is located near upstream facilities.

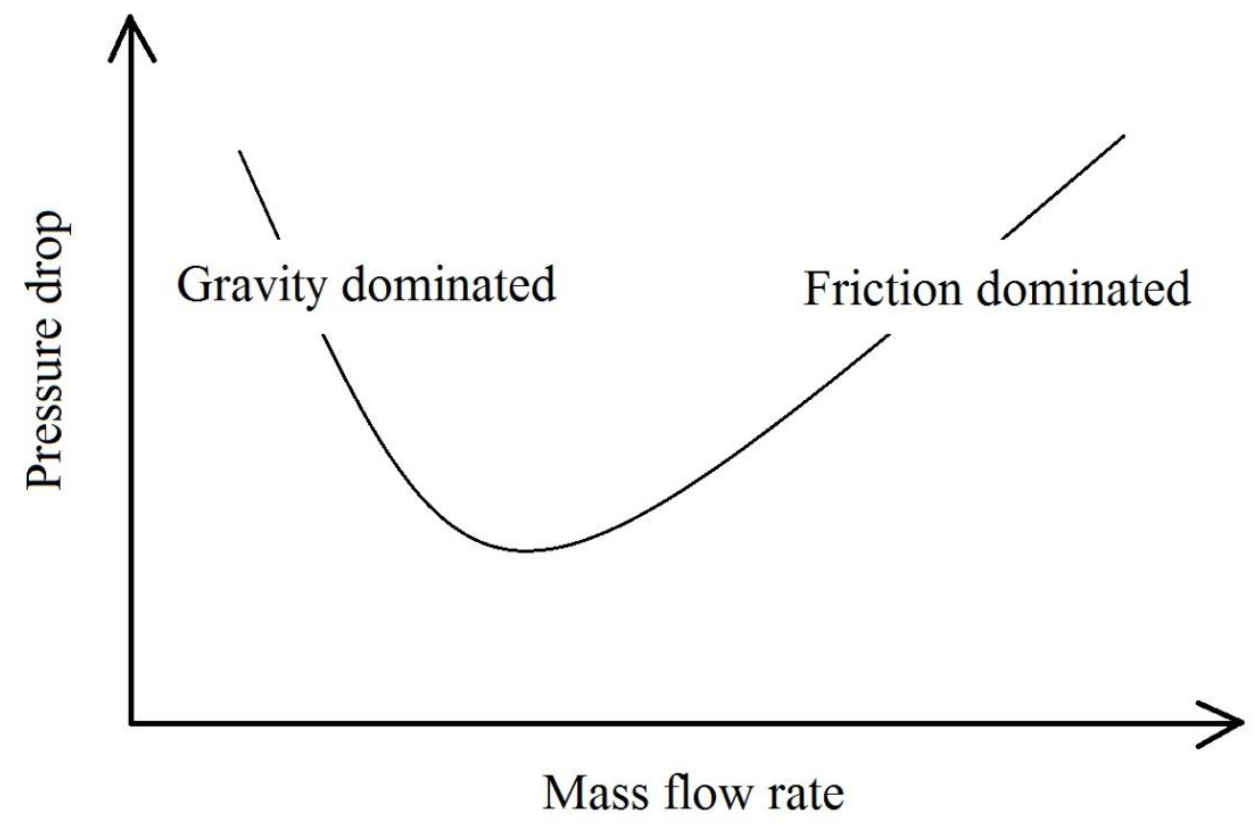

Figure 1. Schematic relationship between pressure drop and flow rate in multiphase flow systems (Bendiksen, 2012)

In offshore oil and gas systems, multiphase flow can exist in both the wellbore and pipeline. The flow in subsea pipelines normally consists of a mixture of three phases including gas, water, and oil. In some cases, a solid phase could also be present due to the reaction of existing phases under the flow conditions. The solid phase may be hydrates (Nicholas et al., 2009), waxes (Singh et al., 2000), or asphaltenes (Kurup et al., 2011), which can sometimes restrict the flow in the system. Therefore, it is important to ensure that the designed pipeline can meet the requirements for the anticipated flow in the system. In order to design a subsea pipeline, the multiphase flow characteristics and behaviour within the pipeline are needed. This could include the phase behaviour, velocity, pressure drop, and temperature of the mixture fluid.

The multiphase flow pattern normally depends on the relative magnitudes of the forces on each phase, such as buoyancy, inertia, and surface-tension forces. These forces are 
dependent on the phase flow rates, pipe diameter, inclination angle, and properties of the phases. With the use of a flow regime map, one can see the flow pattern transition across boundaries. Many studies have identified the appropriate coordinates to effectively show the different flow patterns. The most commonly used flow regime maps are those that are presented based on the gas and liquid superficial velocities, suggested by Taitel et al. (1976). Bratland (2010) presented a graph, showing the possible flow regimes for two-phase flow in horizontal pipes (Fig. 2). As presented in Figure 2, low gas and liquid superficial velocities lead to stratified flow.

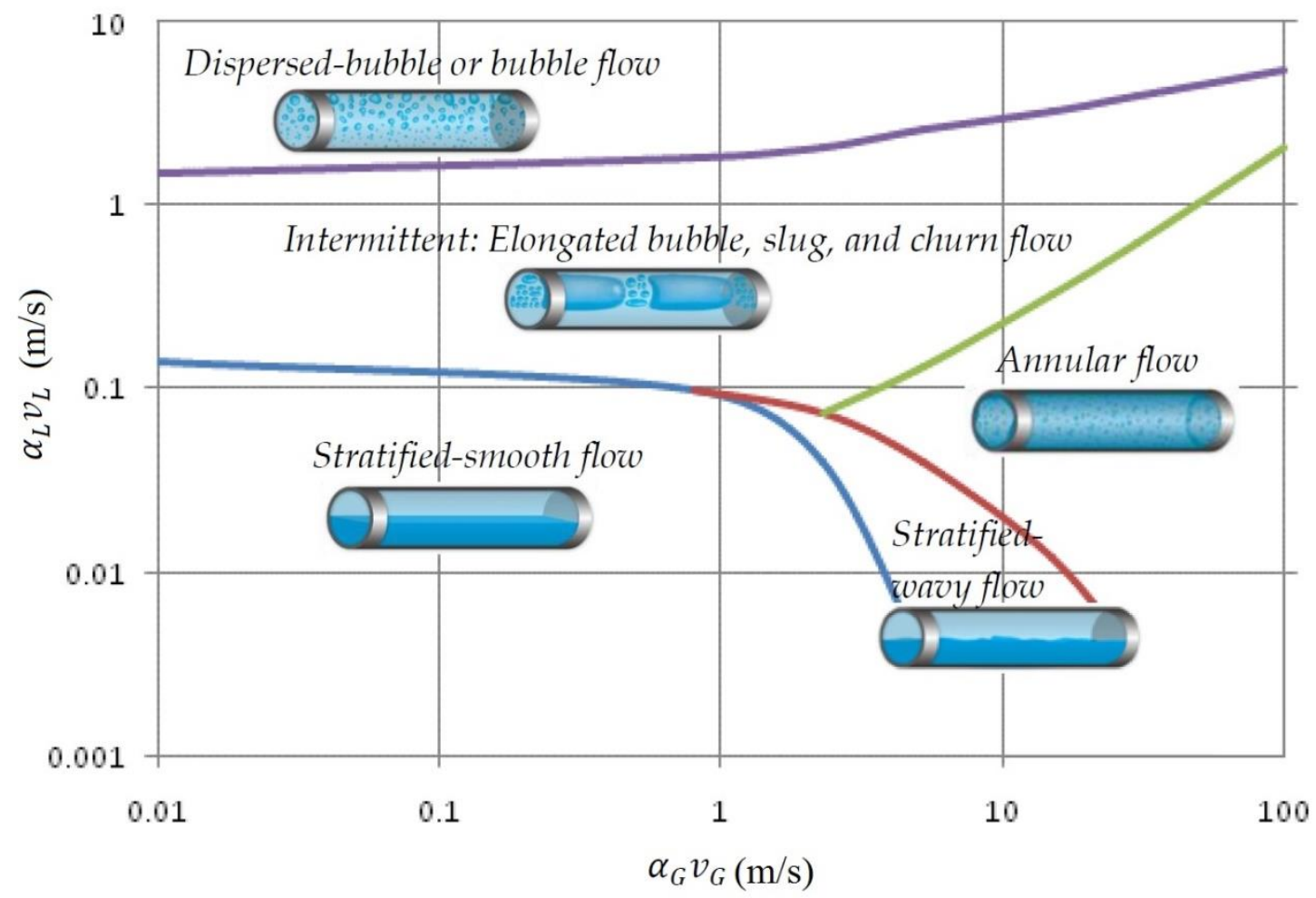

Figure 2. Flow regimes for a two-phase steady-state flow in a horizontal pipe based on gas (X axis) and liquid (Y axis) superficial velocities (Bratland, 2010)

For flow of gas and liquid in horizontal pipes, the flow pattern could have different types depending on the gas and liquid volumetric fractions (Bratland, 2010). Figures 2 and 3 show different flow patterns identified for two-phase gas/liquid flow in horizontal pipes. 


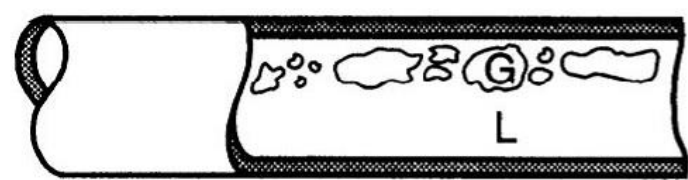

Bubble

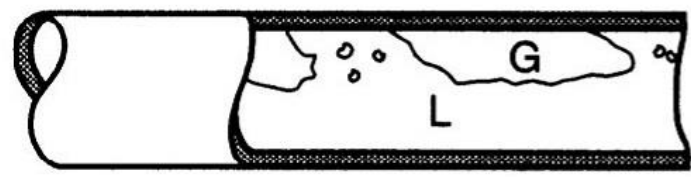

Plug

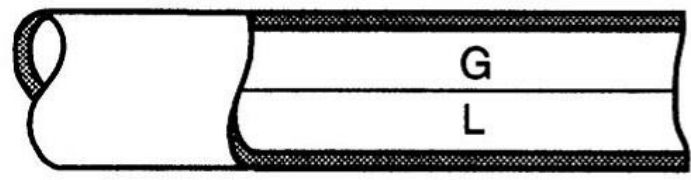

Stratified

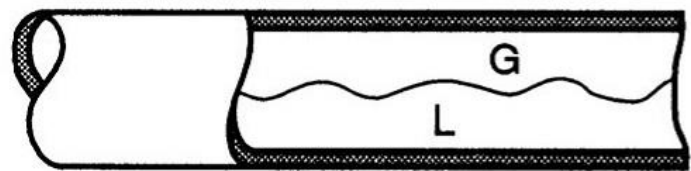

Wavy

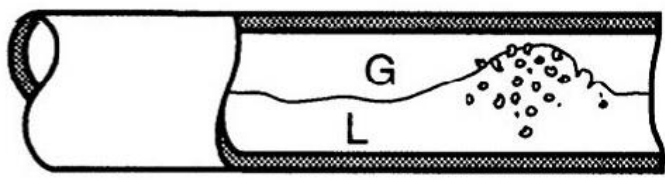

Slug

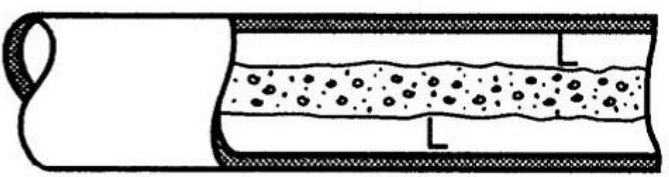

Annular

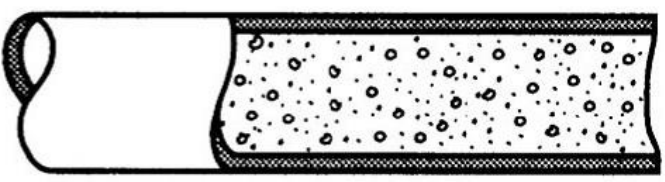

Disperse

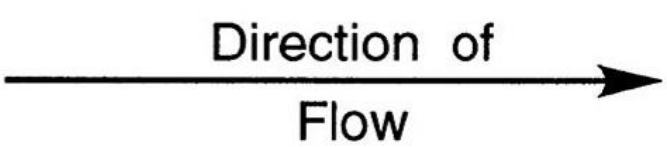

Figure 3. Different types of horizontal flows and flow regimes for gas/liquid flow in a horizontal pipe (Brennen, 2005)

- Bubble flow: Gas bubbles move with the other phase along the top of the conduit.

- Plug flow: Increasing the gas flow rate in the bubble flow can cause interactions between the small bubbles making them into elongated plugs, normally called plug flow.

- Stratified flow: The coalescence of the gas plugs may lead to a continuous gas phase, flowing along the other phase. Low flow rates can lead to a smooth stratified flow, where the interface between the phases is almost a horizontal line.

- Wavy flow: The increase in the gas flow rate could cause instability in the interface and change the flow regime to wavy flow, where the amplitude is higher compared to the smooth stratified flow. 
- Slug flow: Further increases in the amplitude of the interface waves yield large waves in the pipe and change the flow pattern to slug flow. As shown in Fig. 3, small gas bubbles may enter into the liquid phase.

- Annular flow: When the gas phase flow rate is high, the gas core, which includes some liquid droplets, flows through the liquid phase and moves towards the pipe wall. Due to gravity, the thickness of the liquid layer increases towards the bottom of the pipe.

- Dispersed flow: where one phase starts to disperse in the other continuous phase.

The separated-flow model is normally used for stratified flow, where the gas and liquid phases are assumed to flow separately with a connection through the interface between the phases. The cross-sectional area for each phase in this model is equal to the proportion of the pipe cross-sectional area occupied by the phase.

Two-phase flow can also occur for solid/liquid mixtures, consisting of dispersed solid particles in a continuous liquid phase. Depending on the solid phase volume fraction, different flow patterns can exist. Figure 4 shows a range of different flow patterns for solid/liquid mixtures. As the solid particles increase, the flow pattern changes from a homogeneous distribution of dispersed solid particles in the liquid phase, to a heterogeneous distribution with larger particles, to a moving bed of particles, and ultimately to a stationary bed where blockage happens.

To predict multiphase flow, different methods can be used based on the assumptions in their development. In the homogeneous model, it is assumed that the two phases are well mixed to create a single continuous phase, with average properties of the phases. The separated-flow approach assumes each phase flows separately in the pipe. A steady-state momentum equation is developed for gas and liquid phases, and the pressure drop can be 
obtained from either of the two equations. In the transient approach, the conservation equations of mass, momentum and energy are solved simultaneously for each phase.

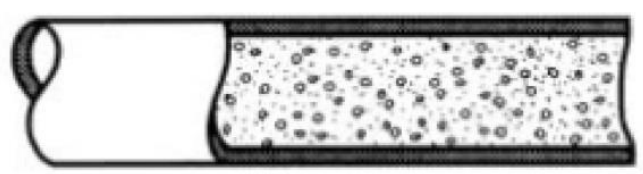

HOMOGENEOUS

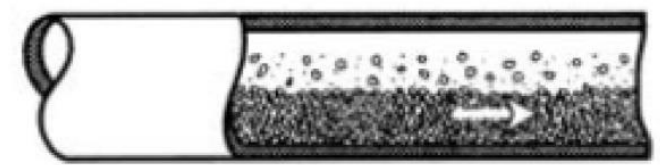

SALTATION : MOVING BED

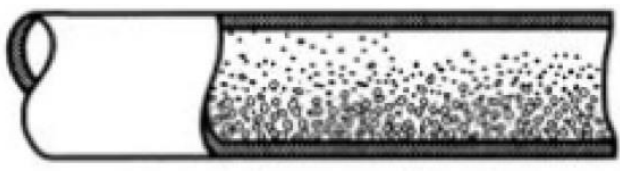

HETEROGENEOUS

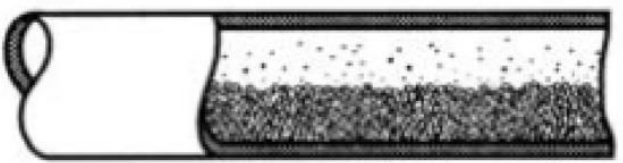

SALTATION : STATIC BED

Figure 4. Flow regimes for slurry flow (solid particles of sand dispersed in water) in a horizontal pipe

(Brennen, 2005)

\subsection{Hydrate formation}

The gas/liquid mixture is typically separated on the platform for pumping and compression and then transported elsewhere. For deepwater operations, a longer subsea tieback (riser) is required, creating flow assurance challenges. One of the most challenging flow assurance problems in oil and gas operations is the presence of hydrate particles (Macintosh, 2000). Gas hydrates are ice-shaped, crystal lattice, solid particles formed by a chemical reaction of water and gases, capable of filling the small or large cavities in the molecular structure of water, forming a hydrogen bond. The solid hydrate may be formed at high pressures and low temperatures (even above the normal melting point of ice) due to weak Van der Waals forces and the hydrogen bonding properties of water. These thermodynamic conditions are often present in subsea pipelines if they are located in deep cold water. The mixture at the wellhead is initially at a high pressure and moderate temperature. As the fluid moves through the subsea system, it cools and creates suitable conditions for hydrate formation. In addition to pressure and temperature, other factors such 
as gas composition, transport effects, and the type of hydrate crystals affect the rate of hydrate formation. (Mork et al., 2001).

Hydrate particles may form and grow along the flow direction in a pipeline both near the wall and along the interface between water, gas or oil, gas. The hydrates may gradually become larger and restrict or block pipelines. The resulting partial or complete plug of a gas pipeline, if not quickly removed, may develop into high-pressure build-up inside the pipe and eventually collapse, causing serious operational problems and safety concerns. This can lead to an oil spill, contamination of ocean water and adverse impact on the habitat of living organisms along the seafloor.

Therefore, it is necessary to accurately predict whether hydrates can block the pipeline system and determine if the rate of hydrate formation is high. This allows mitigation efforts, for example, to calculate the amount of inhibitor needed to remove the hydrate crystals. In order to better predict and prevent hydrate formation and consequent problems in pipelines, different preventative methods can be implemented. The most common methods currently used are thermal, chemical, and mechanical approaches (Kwon et al., 2001). In the thermal method, either the mixture is heated or the system is better insulated to prevent the heat losses from the hydrate formation region. The chemical approach is also widely used where chemicals like methanol are injected into the system to lower the water freezing point. This will shift the hydrate equilibrium conditions to higher pressures and/or lower temperatures, and consequently reduce the possibility of hydrate formation (Figure 5). It is worth noting that for deepwater developments, where the pressure is higher, a large amount of methanol needs to be injected (Creek et al., 2011). The use of some additives is another way to limit the hydrate growth (Mork et al., 2001). 


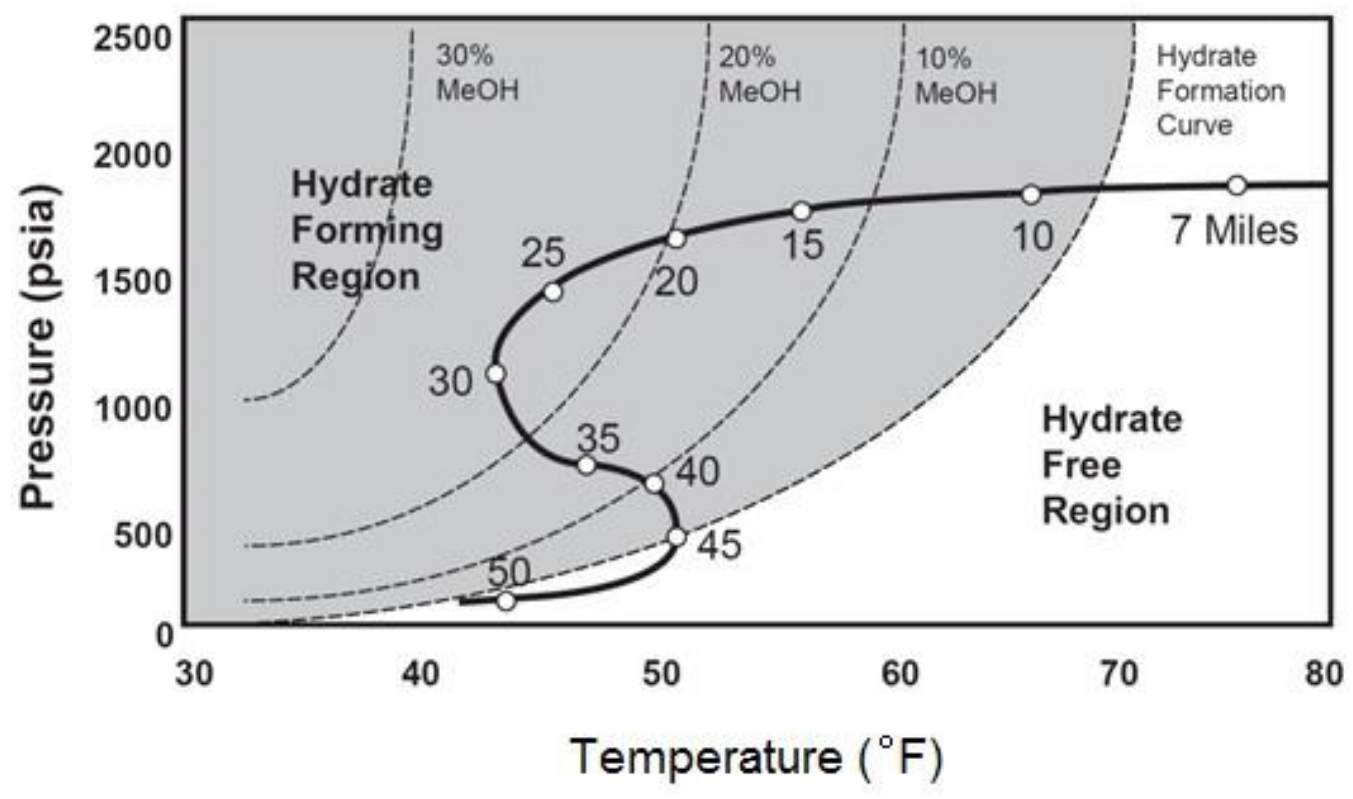

Figure 5. Pressure, temperature, and hydrate equilibrium line for different concentrations of methanol

(Sloan, 2011)

Hydrate equilibrium conditions are the temperatures and pressures in which hydrate particles exist in equilibrium with the gas phase, water, and additive. As shown in Figure 5, such conditions are specified by the curves. Solid hydrates can only form at lower temperatures and higher pressures than the equilibrium conditions (grey area in Figure 5). At a larger distance from the equilibrium curves, there is a higher driving force for hydrate formation.

All of the hydrate prevention methods mentioned above have limitations and require a detailed understanding of multiphase flow to predict the potential and location of hydrate formation in a pipeline (Kwon et al., 2001). It is, therefore, important to develop improved models that can predict the flow characteristics within pipelines, considering the possibility of hydrate formation within the mixture.

There are three general methods to predict the detailed behavior of flows in a multiphase system, including analytical, numerical and experimental methods. Analytical/theoretical methods deal with the mathematical equations and models for the flow. 
In numerical/computational methods, the complexity of the flow is modelled with the use of numerical software programs. The experimental approach acquires measurements of the processes in laboratory studies.

Often laboratory-sized equipment is not able to provide full-scale data. Therefore, analytical and numerical methods are used for real multiphase flow systems, through which extrapolation to a full-scale is predicted. However, the complexity of the flow in a multiphase system is a major challenge in achieving an exact model that can fully describe the flow characteristics in the system. Accurate numerical models require detailed knowledge and high computer power and speed. The flow of the phases in the system is normally turbulent, which significantly increases the complexity of the model. Therefore, the most reasonable way to reduce the complexity of the realistic models is to simplify the problem using appropriate assumptions.

\subsection{Objectives and outline of thesis}

The primary objective of this thesis is to develop a new volume averaged formulation of two-phase and three-phase flows of gas, oil/water, and hydrate particles in subsea pipeline flows. A new semi-analytical model is developed to include the effects of both heat transfer and hydrate growth through the pipeline. Results are presented and new physical insight into the processes is achieved.

Chapter 2 presents a review of past literature in the field. The new problem formulation is presented and modelled in chapter 3, after which solutions are described in chapter 4 and results in chapter 5. Conclusions and recommendations for future research are presented in chapter 6. 


\section{LITERATURE REVIEW}

\subsection{Multiphase flow models}

There have been many past studies on single-phase flow in pipelines (Kwon, 1999; Wylie, 1974). However, only a limited number of studies on multiphase flows in pipelines with hydrates have been conducted. De Henau et al. (1995) developed a one-dimensional transient two-fluid model to predict transient slug flow in pipelines. Their model was validated by comparing the model predictions for gas (or liquid) fractions and pressure drops for steady-state and transient slug flow in pipes to available numerical and experimental data. Tek (1961) considered two immiscible liquids in a single-phase model with averaged mixture properties and presented a model to predict the pressure drop. Gregory and Forgarasi (1985) confirmed a significant difference between theoretical results using averaged mixture properties and experimental results. Liang-Biao Ouyang et al. (2001) developed a simplified model for transient gas-liquid flow in pipes with radial heat flow, and obtained and solved the hyperbolic equations using a finite-difference scheme. Abbaspour et al. (2010) developed a non-isothermal, transient, homogeneous two-phase flow gas pipeline model using a fully implicit finite difference technique.

In three-phase flow, Acikgoz et al. (1992) derived a drill flux model for air/oil/water based on experiments to predict the volume fractions for three-phase flows. Using the momentum equation, Taitel et al. (1995) presented a model for three-phase stratified flow. Khor et al. (1997) improved this model by calculating the shear stresses, and presented a steady-state, three-fluid model. Using empirical correlations, each phase fraction was calculated while neglecting the mass transfer between phases. The aforementioned models are valid only for low velocities in pipelines. For a high gas velocity and high gas void fraction in a gas/liquid two-phase flow, Taitel and Dukler (1976) showed that a continuous liquid film surrounds a core of gas with liquid droplets, which can be categorized as a three- 
phase system of gas, liquid, and droplets. Saito et al. (1978) derived a steady-state three-fluid model and predicted the thermo-hydrodynamic behaviour of annular air/water flow. Tso and Sugawara (1990) used a three-fluid model, called a FIDAS-3DT code, to simulate the axial asymmetric distributions of a liquid film in a horizontal, annular two-phase flow. Kang et al. (1999) developed a non-isothermal three-phase flow model in fluidized beds. Bendiksen et al. (1991) used a dynamic two-phase fluid model, OLGA, and derived the steady-state pressure drop and liquid volume fraction. Bonizzi et al. (2003) presented a numerical approach for one-dimensional transient flow to simulate a stratified flow and a slug flow of liquid/liquid/gas. The two-fluid model consisted of gas and a simplified mixture of two liquid phases. They applied a drift-flux model to predict the motion of the liquid phases relative to each other.

Another approach to calculate the pressure drop in multiphase flow is to modify the pressure gradient equation for a single-phase by assuming that the fluid is a homogeneous mixture of two phases (Brill and Mukherjee, 1999). There are different mixing rules available to calculate the homogeneous fluid properties.

The total pressure drop consists of three different terms including the frictional pressure drop $\left(\Delta P_{f}\right)$, static pressure drop $\left(\Delta P_{s}\right)$, and momentum pressure drop $\left(\Delta P_{m}\right)$ (Thome, 2006). The frictional pressure drop requires evaluation of a two-phase friction factor. The static pressure drop (elevation pressure drop) is dependent on the two-phase mixture density, as well as the inclination of the pipe. For a horizontal pipe, the elevation pressure drop is considered to be zero. The acceleration-related pressure drop is also negligible, as the homogeneous model assumption does not account for the difference in gas and liquid velocities. Since the predicted liquid volume fraction is smaller than its actual value, the homogeneous model assumption under-predicts the pressure drop (Brill and Mukherjee, 1999). 
To consider slip between the gas and liquid phases, past methods were developed based on empirical correlations by calculating the liquid volume fraction as a function of the multiphase flow pattern. Based on the homogeneous fluid model approach, Brill and Mukherjee (1999) arranged the available correlations into three different groups to predict the total pressure drop. In the first group, they considered the correlations that assume an equal velocity for the gas and liquid and do not account for different flow patterns. The properties of the mixture fluid are then calculated based on the input gas/liquid ratio. In the second group, the slip between the gas and liquid phases is considered, but the correlations do not consider the flow patterns. The methods in the second group include correlations for the prediction of the friction factor and liquid holdup.

The methods classified in the third group consider both gas/liquid slip and flow patterns. In the proposed methods in this group, the kinematic pressure drop is dependent on the flow pattern. Therefore, the development of correlations for predicting liquid holdup and friction requires the determination of the flow pattern.

Balasubramaniam et al. (2006) developed another approach for the homogeneous fluid model, which is based on the use of a two-phase correction factor multiplier. This approach estimates the single-phase frictional pressure drop for only the liquid phase, and multiplies the calculated value by a two-phase correction factor.

Lockhart-Martinelli (1949) developed an empirical correlation for the separate flow model. The Lockhart-Martinelli correlation was suggested for horizontal flow of an air/liquid mixture at near-atmospheric pressure. In the development of their model, Lockhart-Martinelli assumed that pressure drops due to flow acceleration and elevation change are negligible, reducing the total pressure drop equation to only a frictional pressure drop. They derived a correlation to calculate the frictional pressure drop in separate two-phase flows. 
The model proposed by Lockhart-Martinelli considers separate correlations for flow pattern, liquid volume fraction and friction factor. However, these characteristics are physically inter-related. To relate these parameters as a part of the model, Bendiksen et al. (1991) developed a transient two-phase flow model including the prior terms.

In the transient two-phase approach, the conservation equations of mass, momentum and energy for each phase are solved simultaneously. The terms in the equations are based on experimental correlations and simplified constitutive relationships for the flow characteristics. Bendiksen et al. (1991) developed three mass transfer equations for the three phases (gas, liquid and droplets) and presented a transient two-phase flow model. The presented by Bendiksen et al. (1991) assumed a two-phase gas/liquid model, where the gas phase consisted of liquid droplets dispersed in the gas phase.

The three phases in Bendiksen's model (1991) are related through the interfacial mass transfer equation, a momentum equation for the combined gas and liquid droplets phases, a momentum equation for the liquid, and an energy equation for the entire system. In the development of this model, the fluid properties such as density, and viscosity, are calculated based on software programs for the fluid properties, such as Multiflash or PVTSim. The data obtained from these programs were used for a range of pressures and temperatures. For a specific pressure and temperature, one can refer to these tables and interpolate the required properties.

In the further development of this model, Bendiksen et al. (1991) assumed four multiphase flow regimes, including stratified, annular, bubble, and slug flow patterns. The transition between the flow regimes is dependent on the gas velocity. They assumed a continuous average gas volume fraction and selected the flow regime with the lowest gas velocity. Due to the dependence of friction factors and wetted perimeters on the flow pattern, Bendiksen et al. (1991) used several semi-experimental correlations to determine the flow 
parameters, such as the average wave height in stratified wavy flow, wall and interfacial friction factors, droplet deposition and entrainment rates, slip velocity between gas and liquid phases, slug bubble velocity, and gas fraction in liquid slugs.

The transient two-phase flow model developed by Bendiksen et al. (1991) has evolved into a commercially available transient multiphase flow model, named OLGAR, which is able to incorporate more than two phases. OLGAR is based on three predefined phases - gas, oil and water - distributed into different mass fields. The mass fields include gas, oil and water as continuous phases, and dispersed droplets within the continuous phases. The oil and water droplets and the gas bubbles are dispersed in the other two continuous phases.

Similar to the two-phase model presented by Bendiksen et al. (1991), the OLGA model considers separate mass conservation equations for each mass field, a combined momentum conservation equation for each layer (i.e., each continuous phase with its dispersed phases), one pressure equation, and one mixture energy conservation equation. The model uses a sequential splitting scheme to solve the conservation equations in subsequent steps for each time step. It starts with the simultaneous solution of the momentum and pressure equations to obtain pressure and velocities, and then solves the set of mass conservation equations to determine phase distributions, and finally the energy equation to solve for the mixture temperature.

\subsection{Hydrate formation and growth models}

The hydrate formation problem has been studied since 1934, when Hammerschmidt found hydrate particles plugging natural gas pipelines. Since the 1930's, researchers have been trying to develop models to understand the performance of kinetic inhibitors. Kvamme (1996) proposed a hydrate formation model where initial hydrate formation occurs at the gas/liquid interface. Kvamme (2002) compared the hydrate growth rate at the two sides of the 
interface and concluded that the hydrate growth rate at the gas side is two orders of magnitude higher than growth at the liquid side. Many studies have been conducted on the rate of hydrate formation after nucleation. The formation rate is normally expressed based on the gas consumption rate. Vysniauskas and Bishnoi (1983) developed a model to measure the rate of methane hydrate formation based on the gas consumption rate in a semi-batch reactor. They suggested that the formation rate depends on various factors, such as gas/liquid interfacial area, pressure, temperature, and subcooling temperature. They also concluded that hydrate formation mostly happens at the gas/liquid interface, where methane concentration is low in the liquid bulk. Later in 1985, they validated their results by conducting experiments with ethane.

Xiao et al. (1998) used a transient multiphase flow simulator, OLGA, to simulate hydrate plug movements. Their results revealed that plug movements are affected by several factors such as pressure differential across a plug, plug size, plug location, and the presence of a liquid phase. Less focus has been placed on studying hydrate growth mechanisms. Sloan (1998) examined the hydrate growth mechanism by studying the mass transfer to the growing surface and growth at the hydrate surface.

Recently, Turner (2005) developed a transient multiphase flow simulator in OLGA to predict the hydrate growth in oil-dominated systems. The work conducted by Nicholas et al. (2008) led to a model to predict the hydrate volume fraction using a wall growth and sloughing model. Joshi (2012) developed a model, CSMHyK-free water-OLGA®, for predicting hydrate formation in high water volume fractions. To predict hydrate equilibrium curves, Sloan (1998) suggested a model based on phase diagrams. The author modified van der Waals and Platteeuw's model and developed a statistical thermodynamics model to determine the hydrate equilibrium curves. 
To my knowledge, none of these studies have considered the impact of hydrates as a separate phase on the flow. Recently, Kwon et al. (2001) developed a transient four-fluid model to predict the transient flow characteristics of gas, condensate, water, and hydrate mixtures in a deep-sea, natural gas pipeline. This model assumes that hydrate particles are constant in size, which is not a realistic condition for an actual oil and gas subsea pipeline. Hydrate particles grow in the flow direction in a pipeline both near the wall (Nicholas, 2008; Rao et al., 2013), and at the interface of liquid and gas. Hydrate formation on pipe walls is normally relatively slow (Lachance et al., 2012), and thus will not be considered in this thesis.

As a result of the gaps in these previous studies, there is a need for improved models that can more accurately predict multiphase flow characteristics, particularly for real conditions in undersea pipelines. Better two-phase models of gas/water and gas/hydrate are required. This objective is the focus of this thesis.

Joshi (2012) conducted an experiment for a gas/water flow and examined how the multiphase flow characteristics may change when hydrates start to form. In a stratified flow, the hydrate formation can quickly change the flow pattern to slug flow. Danielson (2011) used fundamental multiphase flow concepts and developed a model to predict the slug flow. To improve the hydrodynamic slug flow model proposed by Danielson, Zerpa (2013) added a mass balance equation for the hydrate phase and evaluated the hydrates as the third phase. A transient hydrate kinetics model was developed to calculate the hydrate growth rate and mass transfer rate between the phases. Using a transient model instead of the steady-state assumption, Zerpa (2013) calculated the pressure drop and liquid holdup. Heat released from hydrate formation reaction was included in the fluid energy equation to update the temperature distribution. The model was compared against experimental results obtained from experiments in an industrial scale flow loop. 


\section{PROBLEM FORMULATION}

The prediction of multiphase flow can be achieved by solving the conservation equations of mass, momentum, and energy. The conservation equations describe the transport, diffusion and convection of problem variables. The approach to calculate the variables as well as the way to solve the equation discussed in this chapter. To derive the governing equations, several assumptions will be made. Two flow patterns, including stratified flow (see Figure 8) and annular flow are studied, where hydrate particles are assumed to be uniformly dispersed in the liquid phase. To study the variation of flow characteristics along the pipeline, only flow variation in the axial $(x)$ direction will be considered. Viscous dissipation and deposition of hydrate particles into the gas core are neglected. Effects of wall roughness, chemical impurities, and 3-D effects will be neglected.

\subsection{Continuity equation}

The basic continuity equation can be stated as follows:

Accumulated mass + net mass flow into the control volume

$=$ the rate of mass transfer from other phases to phase $N$

$A d x \frac{\partial\left(\alpha_{N} \rho_{N}\right)}{\partial t}+A d x \frac{\partial\left(\alpha_{N} \rho_{N} v_{N}\right)}{\partial x}=\sum_{i}\left(\dot{m}_{N i}+\dot{m}_{N w}\right)$

where $\alpha$ is volume fraction, $\rho$ is density, $A$ is pipe cross-sectional area, $v$ is velocity, $\dot{m}_{N i}$ is the rate of mass transfer from phase $i$ to phase $N$, and $\dot{m}_{N W}$ is the mass transfer rate from the pipe wall to phase $N$. Subscript $N$ in Eq. (2) implies gas $(g)$, water $(w)$, and hydrate $(h)$. The pipe cross-sectional area in this thesis is assumed constant throughout the flow direction. Therefore, dividing the terms in Eq. (2) by $A d x$, the volume of the control volume (see Fig. 6), results in:

$\frac{\partial}{\partial t}\left(\alpha_{N} \rho_{N}\right)+\frac{\partial}{\partial x}\left(\alpha_{N} \rho_{N} v_{N}\right)=\sum_{i}\left(\Gamma_{N i}+\Gamma_{N w}\right)$

where $\Gamma_{N w}$ represents mass transfer rate per unit volume through the wall to phase $N$, and $\Gamma_{N i}$ 
shows mass transfer rate per unit volume from other $i$ to phase $N$. In this study, no mass is assumed to be transferred through the pipe wall. Therefore:

$\Gamma_{N w}=0$

The continuity equation for an individual phase in the control volume is then expressed as follows (Bratland, 2010):

$\frac{\partial}{\partial t}\left(\alpha_{N} \rho_{N}\right)+\frac{\partial}{\partial x}\left(\alpha_{N} \rho_{N} v_{N}\right)=\sum_{i} \Gamma_{N i}$

Also, for the control volume, the sum of the volume fraction of the phases must be equal to one (Eq. 6).

$\sum \alpha_{N}=1$

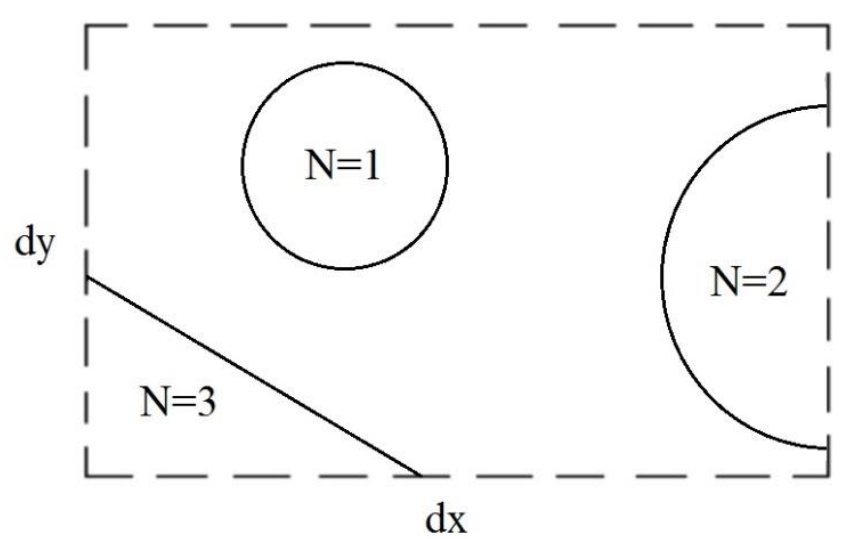

Figure 6. Control volume with three phases (gas, liquid, and solid)

The total mass in the system must be conserved even though there may be phase changes or chemical reactions. In other words:

$\sum_{N} \Gamma_{N}=0$

For a steady-state flow, the continuity equation can be reduced to:

$\frac{d}{d x}\left(\alpha_{N} \rho_{N} v_{N}\right)=\sum_{i} \Gamma_{N i}$

\subsection{Momentum equation}

The momentum equation can be obtained from Newton's second law of motion: 
$\alpha_{N} \rho_{N}(A d x) \times \frac{d v_{N}}{d t}=\sum F_{N}$

where $\sum F_{N}$ represents the sum of all the forces acting on phase $N$ in the control volume. The second term in Eq. (10) shows the rate of change in the phase velocity can be defined as follows:

$d v_{N}=\frac{\partial v_{N}}{\partial t} d t+\frac{\partial v_{N}}{\partial x} d x$

Dividing all the terms in Eq. (11) by $d t$ results in:

$\frac{d v_{N}}{d t}=\frac{\partial v_{N}}{\partial t}+\frac{\partial v_{N}}{\partial x} \frac{d x}{d t}$

where $d x / d t$ represents the velocity of the phase. Therefore:

$\frac{d v_{N}}{d t}=\frac{\partial v_{N}}{\partial t}+v_{N} \frac{\partial v_{N}}{\partial x}$

Replacing $d v_{N} / d t$ in the initial momentum equation, Eq. (10), results in:

$\alpha_{N} \rho_{N} A d x \times\left(\frac{\partial v_{N}}{\partial t}+v_{N} \frac{\partial v_{N}}{\partial x}\right)=\sum F_{N}$

or:

$A d x \times\left(\alpha_{N} \rho_{N} \frac{\partial v_{N}}{\partial t}+\alpha_{N} \rho_{N} v_{N} \frac{\partial v_{N}}{\partial x}\right)=\sum F_{N}$

Using the product rule, one can define the two terms inside of the bracket as follows:

$\frac{\partial\left(\left[\alpha_{N} \rho_{N}\right] \cdot\left[v_{N}\right]\right)}{\partial t}=\alpha_{N} \rho_{N} \frac{\partial v_{N}}{\partial t}+v_{N} \frac{\partial\left(\alpha_{N} \rho_{N}\right)}{\partial t}$

and:

$\frac{\partial\left(\left[\alpha_{N} \rho_{N} v_{N}\right] \cdot\left[v_{N}\right]\right)}{\partial x}=\alpha_{N} \rho_{N} v_{N} \frac{\partial v_{N}}{\partial x}+v_{N} \frac{\partial\left(\alpha_{N} \rho_{N} v_{N}\right)}{\partial x}$

From Eqs. (16) and (17), the two terms inside of the bracket can be defined as:

$$
\begin{aligned}
& \alpha_{N} \rho_{N} \frac{\partial v_{N}}{\partial t}=\frac{\partial\left(\alpha_{N} \rho_{N} v_{N}\right)}{\partial t}-v_{N} \frac{\partial\left(\alpha_{N} \rho_{N}\right)}{\partial t} \\
& \alpha_{N} \rho_{N} v_{N} \frac{\partial v_{N}}{\partial x}=\frac{\partial\left(\alpha_{N} \rho_{N} v_{N}^{2}\right)}{\partial x}-v_{N} \frac{\partial\left(\alpha_{N} \rho_{N} v_{N}\right)}{\partial x}
\end{aligned}
$$


The use of the above two definitions in Eq. (15) will change it to:

$A d x \times\left(\frac{\partial\left(\alpha_{N} \rho_{N} v_{N}\right)}{\partial t}-v_{N} \frac{\partial\left(\alpha_{N} \rho_{N}\right)}{\partial t}+\frac{\partial\left(\alpha_{N} \rho_{N} v_{N}^{2}\right)}{\partial x}-v_{N} \frac{\partial\left(\alpha_{N} \rho_{N} v_{N}\right)}{\partial x}\right)=\sum F_{N}$

Dividing all the terms by $A d x$ changes Eq. (19) to:

$\frac{\partial\left(\alpha_{N} \rho_{N} v_{N}\right)}{\partial t}-v_{N} \frac{\partial\left(\alpha_{N} \rho_{N}\right)}{\partial t}+\frac{\partial\left(\alpha_{N} \rho_{N} v_{N}^{2}\right)}{\partial x}-v_{N} \frac{\partial\left(\alpha_{N} \rho_{N} v_{N}\right)}{\partial x}=\sum R_{N}$

or:

$\frac{\partial\left(\alpha_{N} \rho_{N} v_{N}\right)}{\partial t}+\frac{\partial\left(\alpha_{N} \rho_{N} v_{N}^{2}\right)}{\partial x}=v_{N}\left[\frac{\partial\left(\alpha_{N} \rho_{N}\right)}{\partial t}+\frac{\partial\left(\alpha_{N} \rho_{N} v_{N}\right)}{\partial x}\right]+\sum R_{N}$

where $\sum R_{N}$ represents the sum of all the forces per unit volume on phase $N$. Using the continuity equation, Eq. (5), developed in the previous section, the terms inside of the bracket on the right hand side of Eq. (22) can be replaced by $\sum_{i} \Gamma_{N i}$. Therefore, the momentum equation for phase $N$ is written as:

$\frac{\partial\left(\alpha_{N} \rho_{N} v_{N}\right)}{\partial t}+\frac{\partial\left(\alpha_{N} \rho_{N} v_{N}^{2}\right)}{\partial x}=v_{N} \sum_{i} \Gamma_{N i}+\sum R_{N}$

Expanding the last term in the momentum equation results in:

$\frac{\partial\left(\alpha_{N} \rho_{N} v_{N}\right)}{\partial t}+\frac{\partial\left(\alpha_{N} \rho_{N} v_{N}^{2}\right)}{\partial x}=v_{N} \sum_{i} \Gamma_{N i}+\left(F_{N P g}+F_{N g}+R_{N i}+R_{N w}+S_{N i}+S_{N w}\right)$

where $F_{N P g}, F_{N g}, R_{N i}$, and $R_{N w}$ indicate pressure force, gravity force, friction force from other phases, and friction force from the wall, respectively. $S_{N i}$ represents surface tension from other phases, and $S_{N w}$ represents wall surface tension, both of which are neglected in this thesis. For a horizontal pipe, there is no elevation in the $\mathrm{x}$-direction, meaning that the gravity force is neglected. The pressure force due to the pressure gradient can be calculated as follows:

$F_{N P g}=\frac{A \alpha_{N} P_{N}-A\left(\alpha_{N}+\frac{\partial \alpha_{N}}{\partial x} d x\right)\left(P_{N}+\frac{\partial P_{N}}{\partial x} d x\right)}{A d x}$

Expanding the second term in Eq. (25) simplifies it to: 
$F_{N P g}=\frac{-A \alpha_{N} \frac{\partial P_{N}}{\partial x} d x-A P_{N} \frac{\partial \alpha_{N}}{\partial x} d x-A \frac{\partial \alpha_{N}}{\partial x} \cdot \frac{\partial P_{N}}{\partial x} \cdot(d x)^{2}}{A d x}$

The last two terms are significantly smaller than the first term, and therefore can be neglected. The pressure force term is then simplified to:

$F_{N P g}=-\alpha_{N} \frac{\partial P_{N}}{\partial x}$

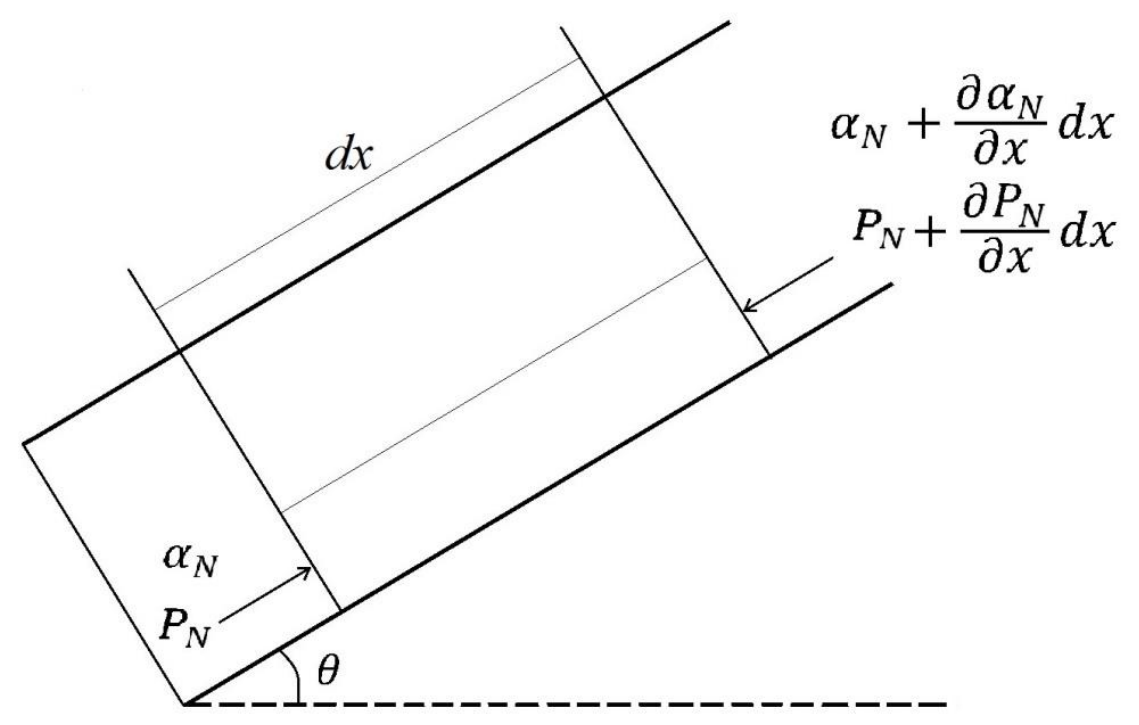

Figure 7. A schematic of pressure force acting on the control volume in a pipe flow

Considering the above terms and assumptions for a horizontal pipe flow, the steadystate momentum equation becomes:

$\frac{\partial}{\partial x}\left(\alpha_{N} \rho_{N} v_{N}^{2}\right)=v_{N} \sum_{i} \Gamma_{N i}-\alpha_{N} \frac{d P_{N}}{d x}+F_{N i}+F_{N w}$

The friction force between the two phases (drag force) in stratified flow is expressed as (Bratland, 2010):

$F_{g l}=\frac{1}{8 A} \rho_{c} f_{g l} p_{g l}\left|v_{g}-v_{l}\right|\left(v_{g}-v_{l}\right)$

where $\rho_{c}, f_{g l}$, and $p_{g l}$ indicate the density of the continuous phase, the interfacial friction factor (drag coefficient), and the gas/liquid interface, respectively. The interfacial friction factor is calculated by the correlation of Petalas and Aziz (1997) as follows: 


$$
f_{g l}=\left(0.004+5 \times 10^{-7} \operatorname{Re}_{s l}\right) F r_{l}^{1.335} \frac{\rho_{l} D g}{\rho_{g} v_{g}^{2}}
$$

where $R e_{s l}$ is the Reynolds number based on the superficial velocity $\left(\alpha_{l} v_{l}\right), D$ is the inside pipe diameter, and $F r_{l}$ is the Froude number for liquid. $F r_{l}$ is defined as follows:

$F r_{l}=\frac{v_{l}}{\sqrt{g h_{l}}}$

where $h_{l}$ is the liquid height, which is the difference between the interface and the center of the liquid phase. Liquid height can be defined by the correlation proposed by De Henau et al. (1995):

$h_{l}=\left[-\frac{1}{2} \cos \left(\pi-\frac{\beta}{2}\right)+\frac{1}{3 \pi \alpha_{l}} \sin ^{3}\left(\pi-\frac{\beta}{2}\right)\right] D \cos \theta$

The gas phase angle $(\beta)$, as shown in Fig. 8 , is estimated by Biberg's correlation (1999) as follows:

$$
\beta=2 \pi-2\left[\pi \alpha_{l}+\left(\frac{3 \pi}{2}\right)^{\frac{1}{3}}\left(1-2 \alpha_{l}+\alpha_{l}^{\frac{1}{3}}-\left(1-\alpha_{l}\right)^{\frac{1}{3}}\right)\right]
$$

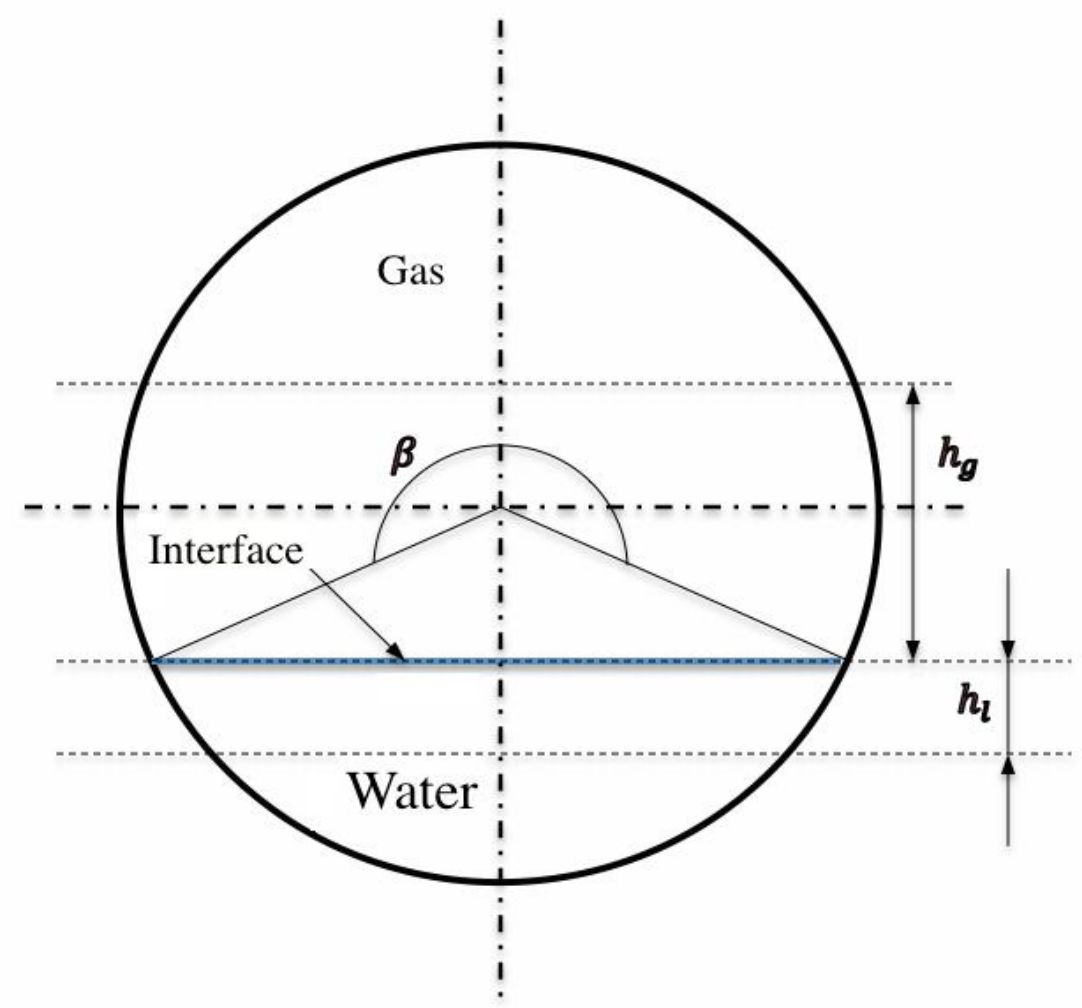

Figure 8. Gas/water stratified flow 
The gas/liquid interface is calculated by:

$p_{g l}=D \sin \frac{\beta}{2}$

The particle drag force per unit volume for dispersed flow can be written as:

$R_{g h}=\frac{3 \alpha_{h} \rho_{g} f_{g h}}{4 D_{h}}\left|v_{g}-v_{h}\right|\left(v_{g}-v_{h}\right)$

where $f_{g h}$ and $D_{h}$ represent the drag force coefficient and the average particle's diameter, respectively. Yuen and Chen (1976) reported that the drag coefficient for small particles is close to that for solid spheres. Cheng's (2009) correlation states that the drag force coefficient for spheres can be calculated by:

$f_{g h}=\frac{24}{R e_{g h}}\left(1+0.27 R e_{g h}\right)^{0.43}+0.47\left[1-\exp \left(-0.04 R e_{g h}^{0.38}\right)\right]$

where $R e_{g h}$ is the hydrate particle Reynolds number defined as:

$R e_{g h}=\frac{\rho_{g}\left|v_{g}-v_{h}\right| D_{h}}{\mu_{g}}$

where $\mu_{g}$ is dynamic viscosity for the gas phase.

According to Bratland (2010), the wall friction force for gas/liquid stratified flow can be written as:

$F_{N w}=-\frac{1}{8 A} \rho_{N} f_{N w} p_{N w} v_{N}\left|v_{N}\right|$

where the subscript $N$ represents gas and liquid. Also, $f_{N w}$ is the Darcy-Weisbach friction factor, and it can be calculated as follows:

$f_{N w}=\frac{64}{R e_{N}}$

where $R e_{N}$ indicates Reynolds number for phase $N$. This correlation is based on the HagenPoiseuille's law. It is only valid for laminar conditions $\left(R e_{N}<2300\right)$. For turbulent flows $\left(R e_{N}>4000\right)$, the following iterative expression proposed by Colebrook and White equation (1937) can be used: 
$\frac{1}{\sqrt{f_{N w}}}=-2 \log \left(\frac{\varepsilon / D}{3.7}+\frac{2.51}{R e_{N} \sqrt{f_{N w}}}\right)$

where $\varepsilon$ is wall roughness height and $\varepsilon / D$ is relative roughness. Haaland proposed an alternative approximation to the Colebrook correlation as follows:

$\frac{1}{\sqrt{f_{N w}}}=-1.8 \log \left[\left(\frac{\varepsilon / D}{3.7}\right)^{1.11}+\frac{6.9}{R e_{N}}\right]$

For smooth pipes, Petukhov (1970) developed the following correlation:

$f_{N w}=\left(0.79 \ln R e_{N}-1.64\right)^{-2}$

which is valid for $3000<R e_{N}<5 \times 10^{6}$.

In the wall friction equation, $p_{N w}$ describes the wetted perimeter by phase $N$. According to Fig. 8, $p_{g w}$ and $p_{l w}$ can be calculated as follows:

$p_{g w}=\beta \frac{D}{2}$

$p_{l w}=(2 \pi-\beta) \frac{D}{2}$

To obtain the friction force, one can write a force balance equation on a control volume as follows:

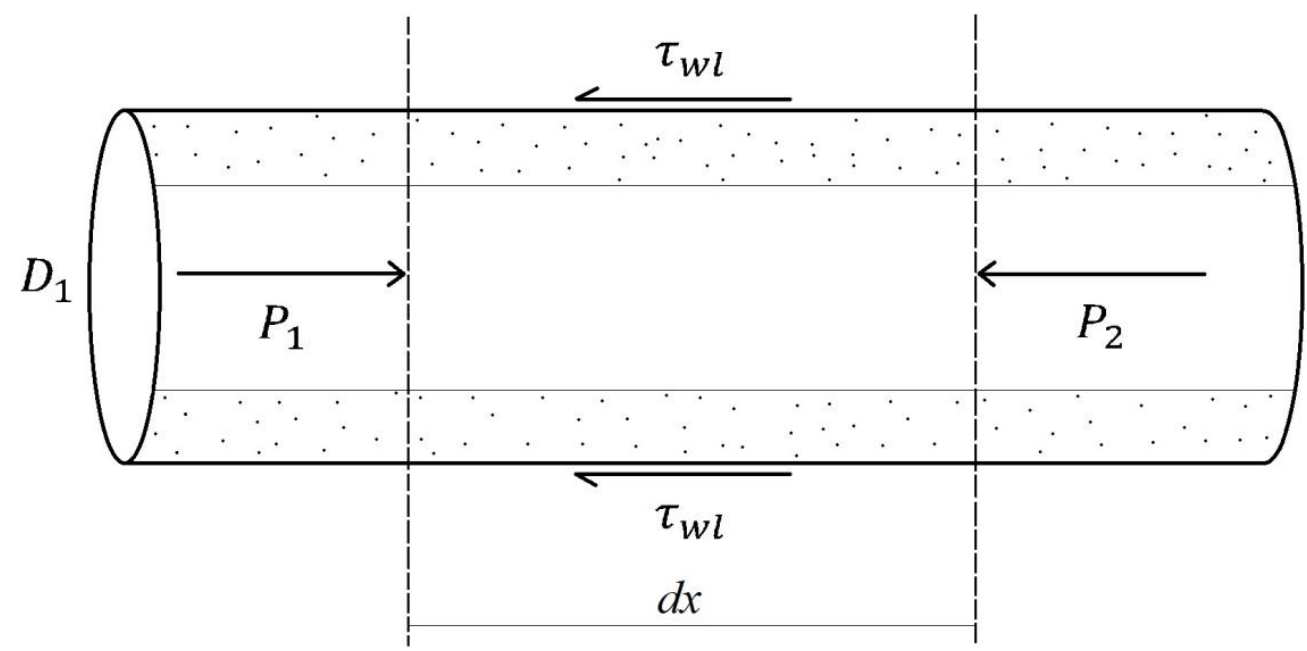

Figure 9. Friction force on the control volume

$\tau_{w l}\left(\pi D_{1} d x\right)=\left(P_{2}-P_{1}\right)\left(\frac{\pi D_{1}^{2}}{4}\right)\left(\frac{\alpha_{l}}{\alpha_{h}+\alpha_{l}}\right)$ 
where the pressure difference $\left(P_{2}-P_{1}\right)$ can be calculated as follows:

$\left(P_{2}-P_{1}\right)=f_{l w} \frac{d x}{D_{1}} \rho_{l} \frac{v_{l}^{2}}{2}$

Replacing Eq. (46) in Eq. (45) results in:

$\tau_{w l}\left(\pi D_{1} d x\right)=f_{l w} \frac{d x}{D_{1}} \rho_{l} \frac{v_{l}^{2}}{2}\left(\frac{\pi D_{1}^{2}}{4}\right)\left(\frac{\alpha_{l}}{\alpha_{h}+\alpha_{l}}\right)$

Dividing both sides of Eq. (47) by the volume of the control volume, $\left(\pi D_{1}^{2} / 4\right) d x$, results in:

$F_{l w}=\frac{1}{2 D_{1}}\left(\frac{\alpha_{l}}{\alpha_{h}+\alpha_{l}}\right) \rho_{l} f_{l w} v_{l}\left|v_{l}\right|$

In general, the friction force for annular dispersed flow with hydrate particles is expressed as (Kwon et al., 2001):

$F_{N w}=\frac{1}{2 D}\left(\frac{\alpha_{N}}{\alpha_{g}+\alpha_{h}}\right) \rho_{N} f_{N w} v_{N}\left|v_{N}\right|$

The total pressure drop consists of a frictional pressure-drop $\left(\Delta P_{f}\right)$, static pressure drop $\left(\Delta P_{s}\right)$ due to elevation change, and momentum pressure drop $\left(\Delta P_{m}\right)$. The sum of these three pressure drops yields the total pressure drop as follows (Thome, 2006):

$\Delta P_{t o t}=\Delta P_{f}+\Delta P_{s}+\Delta P_{m}$

For a horizontal pipe, $\Delta P_{s}$ is negligible as no change occurs in the static head. $\Delta P_{m}$ is the momentum-based pressure difference at the inlet and outlet. As there is no condensation/evaporation, and the velocity does not change significantly from the inlet to the outlet, $\Delta P_{m}$ is negligible compared to the frictional pressure-drop (Thome, 2006). Therefore, the total pressure drop is determined from the frictional pressure drop.

\subsection{Energy equation}

The conservation of energy equation describes the first law of thermodynamics. The energy equation consists of energy terms, heat transfer, and work in the system. Assuming that phases have an equal temperature, the energy equation is given by (Kwon et al., 2001): 


$$
\begin{aligned}
& \sum_{N}\left(\alpha_{N} \rho_{N} C_{P N}\right) \frac{\partial T}{\partial t}+\sum_{N}\left(\alpha_{N} \rho_{N} v_{N} C_{P N}\right) \frac{\partial T}{\partial x}=\sum_{N}\left(\alpha_{N} \rho_{N} \eta_{N} C_{P N}\right) \frac{\partial P}{\partial t}+\sum_{N}\left(\alpha_{N} \rho_{N} v_{N} \eta_{N} C_{P N}\right) \frac{\partial P}{\partial x} \\
& +\sum_{N}\left(\alpha_{N} \rho_{N} \frac{\partial}{\partial t}\left(\frac{P}{\rho_{N}}\right)\right)+\sum_{N}\left(\alpha_{N} \rho_{N} v_{N} \frac{\partial}{\partial x}\left(\frac{P}{\rho_{N}}\right)\right)-\frac{P}{A} \frac{\partial}{\partial x} \sum_{N}\left(\alpha_{N} v_{N} A\right)-\sum_{N}\left(\Gamma_{N}\left(h_{N}-\frac{P}{\rho_{N}}\right)\right)+\dot{Q}
\end{aligned}
$$

where $C_{P N}$ is the heat capacity of phase $N$ at constant pressure, $T$ is the fluid average temperature, $\eta$ is the Joule-Thomson factor, and $h_{N}$ is the specific enthalpy of phase $N$. For steady-state liquid/gas flow without mass transfer between phases, the energy equation is reduced to:

$$
\sum_{N}\left(\alpha_{N} \rho_{N} v_{N} C_{P N}\right) \frac{\partial T}{\partial x}=\sum_{N}\left(\alpha_{N} \rho_{N} v_{N} \eta_{N} C_{P N}\right) \frac{\partial P}{\partial x}+\sum_{N}\left(\alpha_{N} \rho_{N} v_{N} \frac{\partial}{\partial x}\left(\frac{P}{\rho_{N}}\right)\right)+\dot{Q}
$$

The last term in the energy equation, $\dot{Q}$, represents heat transfer rate per unit volume added to the fluid, which is approximated as follows:

$$
\dot{Q}=2 U \frac{r_{o}}{r_{i}^{2}}\left(T_{o}-T_{i}\right)
$$

where $r_{i}$ is the inside pipe radius, $r_{o}$ is the outside pipe radius, $U$ is the overall heat transfer coefficient, and $T_{o}$ and $T_{i}$ represent the surrounding temperature and the average fluid temperature inside the pipe, respectively. The overall heat transfer coefficient can be calculated by:

$\frac{1}{U}=\frac{1}{h_{i}}+\frac{r_{i}}{k} \ln \frac{r_{i}}{r_{o}}+\frac{r_{i}}{h_{o} r_{o}}$

where $k$ represents conductivity of the pipe, and $h_{i}$ and $h_{o}$ are the convective heat transfer coefficients for the inside and outside of the pipe, respectively. Depending on the Reynolds number of the mixture fluid, $h_{i}$ and $h_{o}$ can be estimated for laminar or turbulent flows. Bergman et al. (2011) suggested that for laminar flow in a pipe with constant surface temperature $\left(R e_{f}<2300\right), h_{i}$ can be calculated based on the following correlation:

$$
N u=3.66
$$

where $\mathrm{Nu}$ represents the Nusselt number, defined as follows: 
$N u=\frac{h_{i} D}{k_{f}}$

In Eq. (56) $k_{f}$ is the fluid conductivity and should be evaluated at the mean fluid temperature. Gnielinski (1976) suggested the following correlation for turbulent flow:

$N u=\frac{(f / 8)\left(R e_{f}-1000\right) P r_{f}}{1.07+12.7(f / 8)^{1 / 2}\left(\operatorname{Pr}_{f}^{2 / 3}-1\right)}$

where $f$ is the friction factor stated previously. Gnielinski's correlation is valid under the following conditions:

$2300 \leq R e_{f} \leq 5 \times 10^{6}$

$0.5 \leq P r_{f} \leq 2000$

For the convection heat transfer coefficient for solid sphere hydrate particles, Whitaker (1972) recommended a correlation of the form:

$h_{i}=\frac{k_{h}}{D_{h}}\left[2+\left(0.4 R e_{h}^{1 / 2}+0.06 \operatorname{Re}_{h}^{2 / 3}\right) \operatorname{Pr}_{f}^{0.4}\left(\frac{\mu_{f}}{\mu_{f s}}\right)\right]$

where $k_{h}$ is thermal conductivity of hydrate particles, $R e_{h}$ is the Reynolds number of solid particles, and $P r_{f}$ is the Prandtl number of the continuous phase (gas phase). Also, $\mu_{f}$ and $\mu_{f s}$ represent dynamic viscosity of the gas phase at the fluid temperature and wall temperature, respectively. The fluid convection coefficient is finally estimated based on the volume averaging method, using each phase convective coefficient.

\subsection{Hydrate formation and growth}

Predictions of the hydrate formation location and growth rate in a pipeline are important in the oil and gas industry. Identifying where the plugs start to grow can help to identify more efficient techniques to prevent hydrate growth. In order to determine the hydrate formation rate, it is necessary to predict the pressure and temperature conditions along the pipeline. In this study, the pressure profile along the pipeline is determined by 
calculating the pressure drop in multiphase flow. The temperature distribution is estimated by solving the energy equation.

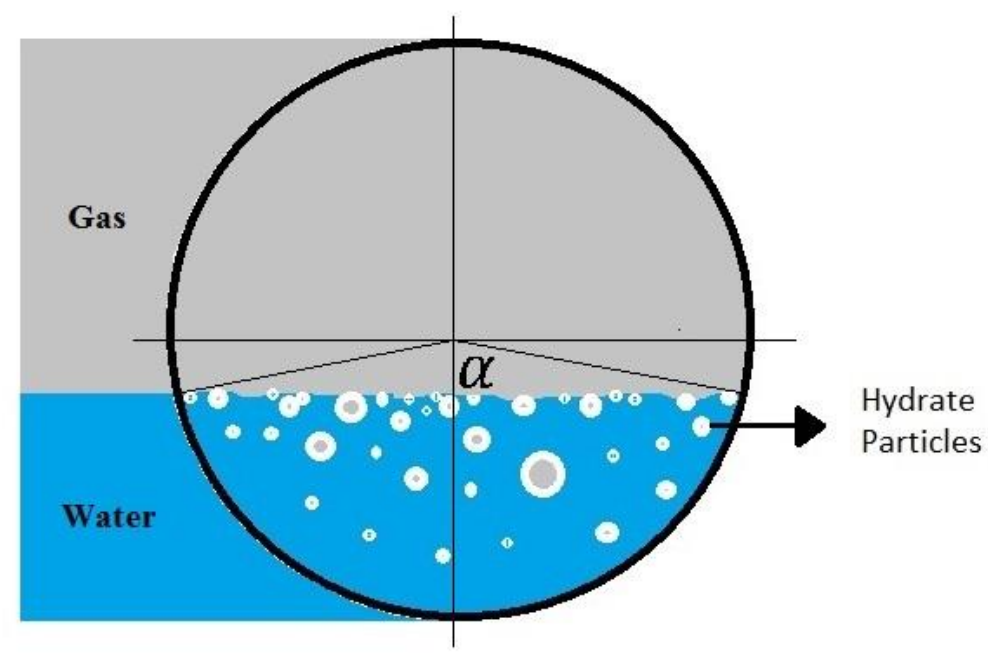

Figure 10. Cross-section of the pipe for a stratified flow pattern

Figure 10 shows a schematic of the three phases in the pipeline in a stratified model. Formation of the hydrate particles adds the hydrate volume fraction to the equations. The sum of the volume fractions will yield:

$\alpha_{g}+\alpha_{l}+\alpha_{h}=1$

Also, the average velocity for the mixture can be calculated as follows:

$\rho v=\alpha_{g} \rho_{g} v_{g}+\alpha_{l} \rho_{l} v_{l}+\alpha_{h} \rho_{h} v_{h}$

where $\rho$ represents the mixture density, obtained based on the densities and volume fractions of phases as follows:

$\rho=\alpha_{g} \rho_{g}+\alpha_{l} \rho_{l}+\alpha_{h} \rho_{h}$

Hydrate particles are assumed to grow within the liquid phase (Zerpa et al., 2012), and not on the wall. It is assumed there is no drag force between gas and hydrates, while liquid carries the hydrates. Because of the lower density of hydrate particles compared to water, they may rise to the top of the water phase and below the gas phase. Joshi et al. (2013) revealed that hydrates are dispersed in the liquid phase unless a high volume fraction of the hydrate phase restricts the flow in the system. 
Rao (2013) proposed a model for the consumption of the water phase due to hydrate formation. For a control volume, the mass transfer between the water and hydrate phase can be calculated by:

$\Gamma_{l h}=\frac{n_{h}}{\Delta x} \frac{d m_{g a s}}{d t}$

where $\Gamma_{l h}$ and $n_{h}$ represent the liquid phase consumption rate and the hydration number, respectively (Sloan and Koh, 2008). The last term in the above equation, $\frac{d m_{\text {gas }}}{d t}$, is the gas consumption rate due to hydrate formation. It is determined by Turner's intrinsic kinetics equation (2005):

$-\frac{d m_{g a s}}{d t}=k_{1} \exp \left(\frac{k_{2}}{T}\right) A_{s} \Delta T_{\text {sub }}$

where $k_{1}$ and $k_{2}$ are the intrinsic rate constants, and $A_{s}$ represents the interfacial area between water and gas. According to experimental data of Vysniauskas and Bishnoi (1983), the intrinsic rate constants are as follows:

$k_{1}=7.3548 \times 10^{17}$

$k_{2}=-13600 \mathrm{~K}$

For a stratified flow, $A_{s}$ can be defined as a function of radius (r) and $\alpha$ as follows (Rao, 2013):

$A_{s}=2 r(\sin \alpha) \Delta x$

where $\Delta x$ is a small section of the pipe and $\alpha$ is the wetted angle of the liquid phase, expressed by Biberg's (1999) equation (Fig. 10):

$\alpha \cong \frac{\pi}{2}\left(1-\left(1-\alpha_{l}\right)^{1 / 3}+\alpha_{l}^{1 / 3}\right)$

where $\alpha_{l}$ is the volume fraction of the liquid phase. Also, $\Delta T_{s u b}$ (subcooling) in Eq. (65) is the driving mechanism behind the hydrate formation. It is obtained by the difference of the fluid temperature $(T)$ and the equilibrium temperature $\left(T_{h y d, e q}\right)$. Hydrates are formed when 
the fluid temperature drops below the equilibrium temperature. In order for the hydrates to grow, $\Delta T_{\text {sub }}$ must be positive (Rao, 2013).

In the next chapter, the solution method will be described. A numerical solution of the previous reduced form of conservation equations will be presented. 


\section{SOLUTIONS OF CONSERVATION EQUATIONS}

\subsection{Introduction}

In order to solve the nonlinear conservation equations (continuity, momentum, and energy), a Newton-Raphson method was used. The method requires one initial value for each unknown to start the algorithm (Urroz, 2004). Like other numerical methods, the NewtonRaphson algorithm obtains an approximate solution of the discretized equations. A tolerance level is set and iterations continue until equations are solved within the allowed tolerance or error.

To simulate three-phase conditions, including the hydrate phase, two-phase models of gas/liquid were developed first. In the two-phase stratified model, the liquid and gas phases are linked via friction, total cross-sectional area, and pressure. It is assumed that the interface is approximately flat and horizontal, which is responsible for low gas velocities. Using the Newton-Raphson method, the governing equations are solved simultaneously for velocity, volume fraction, pressure, and temperature along the pipeline.

The system of $n$ nonlinear equations with $n$ unknowns can be written as:

$f_{1}\left(x_{1}, x_{2}, \ldots, x_{n}\right)=0$

$f_{2}\left(x_{1}, x_{2}, \ldots, x_{n}\right)=0$

$\vdots$

$f_{n}\left(x_{1}, x_{2}, \ldots, x_{n}\right)=0$

The system of equations represented in Eq. (70) can be written in a single equation as:

$f(x)=0$

where $x$ is an $n \times 1$ matrix containing $n$ independent variables and $f$ is an $n \times 1$ matrix with $n$ functions:

$x=\left[\begin{array}{c}x_{1} \\ x_{2} \\ \vdots \\ x_{n}\end{array}\right]$ 
$f(x)=\left[\begin{array}{c}f_{1}(x) \\ f_{2}(x) \\ \vdots \\ f_{n}(x)\end{array}\right]=\left[\begin{array}{c}f_{1}\left(x_{1}, x_{2}, \ldots, x_{n}\right) \\ f_{2}\left(x_{1}, x_{2}, \ldots, x_{n}\right) \\ \vdots \\ f_{n}\left(x_{1}, x_{2}, \ldots, x_{n}\right)\end{array}\right]$

In order to solve the above systems of nonlinear equations, the Jacobian matrix is used, which is defined as:

$$
J=\frac{\partial\left(f_{1}, f_{2}, \ldots, f_{n}\right)}{\partial\left(x_{1}, x_{2}, \ldots, x_{n}\right)}=\left[\begin{array}{cccc}
\frac{\partial f_{1}}{\partial x_{1}} & \frac{\partial f_{1}}{\partial x_{2}} & \ldots & \frac{\partial f_{1}}{\partial x_{n}} \\
\frac{\partial f_{2}}{\partial x_{1}} & \frac{\partial f_{2}}{\partial x_{2}} & \ldots & \frac{\partial f_{2}}{\partial x_{n}} \\
\vdots & \vdots & \ddots & \vdots \\
\frac{\partial f_{n}}{\partial x_{1}} & \frac{\partial f_{n}}{\partial x_{2}} & \cdots & \frac{\partial f_{n}}{\partial x_{n}}
\end{array}\right]
$$

Considering $x=x_{0}$ as the initial guess, the next approximations are calculated by:

$x_{n+1}=x_{n}-\Delta x_{n}$

where

$$
\Delta x_{n}=J^{-1} \cdot f\left(x_{n}\right)
$$

To obtain accurate approximations, a very small residual $\left(\Delta x_{n}\right)$ between the consecutive solutions should be set, and the iterations should continue until the desired convergence criterion is achieved.

In order to calculate the elements in the Jacobian matrix, analytical or numerical methods can be implemented. The analytical method deals with the use of derivative correlations to find elements in the matrix. However, in the numerical method, the Jacobian matrix is approximated through the definition of the derivative as follows:

$\frac{\partial f_{i}}{\partial x_{j}}=\frac{f_{1}\left(x_{1}, x_{2}, \ldots, x_{j}+\Delta x, \ldots, x_{n}\right)-f_{1}\left(x_{1}, x_{2}, \ldots, x_{n}\right)}{\Delta x}$

where $\Delta x$ represents a very small increment in the independent variables. In this thesis the Jacobian matrix is obtained through the analytical method. The required equations are written in symbolic form, and then differentiated with respect to independent variables to form the elements in the Jacobian matrix. 


\subsection{Two-phase flow solutions}

Considering no mass transfer between liquid and gas phases, the steady-state continuity equation, Eq. (8), for the gas phase is reduced to:

$\frac{d}{d x}\left(\alpha_{g} \rho_{g} v_{g}\right)=0$

As an assumption, the mass flow rates of the gas and liquid phases are constant along the pipeline. Therefore, integrating both sides and multiplying by $A_{g}$ results in:

$\alpha_{g} \rho_{g} v_{g} A_{g}=\dot{m}_{g}$

where $\dot{m}_{g}$ is the gas phase mass flow rate. Following the same procedure for the liquid phase, one can write:

$\alpha_{l} \rho_{l} v_{l} A_{l}=\dot{m}_{l}$

where $\dot{m}_{l}$ represents the mass flow rate of the liquid phase.

Convective term in the momentum equation can be written as:

$\frac{\partial\left(\alpha_{g} \rho_{g} v_{g}^{2}\right)}{\partial x}=\alpha_{g} \rho_{g} v_{g} \frac{\partial v_{g}}{\partial x}+v_{g} \frac{\partial\left(\alpha_{g} \rho_{g} v_{g}\right)}{\partial x}$

Based on the steady-state continuity equation, Eq. (78), the last term in Eq. (81) is equal to zero. Therefore, the convective term can be written as:

$\frac{\partial\left(\alpha_{g} \rho_{g} v_{g}^{2}\right)}{\partial x}=\alpha_{g} \rho_{g} v_{g} \frac{\partial v_{g}}{\partial x}$

and for the liquid phase:

$\frac{\partial\left(\alpha_{l} \rho_{l} v_{l}^{2}\right)}{\partial x}=\alpha_{l} \rho_{l} v_{l} \frac{\partial v_{l}}{\partial x}$

Assuming an initial value for temperature, the governing equations are solved by the Newton-Raphson algorithm. In the presented two-phase model, these equations represent the continuity, momentum, and volume fraction equations with a discrete grid spacing, $\Delta x(5 \mathrm{~m})$, as follows:

$f_{1}(x)=\alpha_{g} \rho_{g} v_{g} A_{g}-\dot{m}_{g}=0$ 
$f_{2}(x)=\alpha_{l} \rho_{l} v_{l} A_{l}-\dot{m}_{l}=0$

$f_{3}(x)=\alpha_{g}+\alpha_{l}-1=0$

$f_{4}(x)=\alpha_{g} \rho_{g} v_{g} \frac{\partial v_{g}}{\partial x}+\alpha_{g} \frac{d P}{d x}-F_{l g}-F_{w g}=0$

$f_{5}(x)=\alpha_{l} \rho_{l} v_{l} \frac{\partial v_{l}}{\partial x}+\alpha_{l} \frac{d P}{d x}-F_{g l}-F_{w l}=0$

The system of equations represented above can be written in a single equation as:

$f(x)=0$

where $x$ is a $5 \times 1$ matrix containing 5 independent variables and $f$ is an $5 \times 1$ matrix with 5 functions:

$x=\left[\begin{array}{l}x_{1} \\ x_{2} \\ x_{3} \\ x_{4} \\ x_{5}\end{array}\right]=\left[\begin{array}{c}\alpha_{g} \\ \alpha_{l} \\ v_{g} \\ v_{l} \\ d P \\ \frac{d x}{d x}\end{array}\right]$

$f(x)=\left[\begin{array}{l}f_{1}(x) \\ f_{2}(x) \\ f_{3}(x) \\ f_{4}(x) \\ f_{5}(x)\end{array}\right]$

In order to solve the above systems of nonlinear equations, the Jacobian matrix is developed as follows:

$$
J=\frac{\partial f}{\partial x}=\frac{\partial\left(f_{1}, f_{2}, f_{3}, f_{4}, f_{5}\right)}{\partial\left(x_{1}, x_{2}, x_{3}, x_{4}, x_{5}\right)}=\left[\begin{array}{lllll}
\frac{\partial f_{1}}{\partial x_{1}} & \frac{\partial f_{1}}{\partial x_{2}} & \frac{\partial f_{1}}{\partial x_{3}} & \frac{\partial f_{1}}{\partial x_{4}} & \frac{\partial f_{1}}{\partial x_{5}} \\
\frac{\partial f_{2}}{\partial x_{1}} & \frac{\partial f_{2}}{\partial x_{2}} & \frac{\partial f_{2}}{\partial x_{3}} & \frac{\partial f_{2}}{\partial x_{4}} & \frac{\partial f_{2}}{\partial x_{5}} \\
\frac{\partial f_{3}}{\partial x_{1}} & \frac{\partial f_{3}}{\partial x_{2}} & \frac{\partial f_{3}}{\partial x_{3}} & \frac{\partial f_{3}}{\partial x_{4}} & \frac{\partial f_{3}}{\partial x_{5}} \\
\frac{\partial f_{4}}{\partial x_{1}} & \frac{\partial f_{4}}{\partial x_{2}} & \frac{\partial f_{4}}{\partial x_{3}} & \frac{\partial f_{4}}{\partial x_{4}} & \frac{\partial f_{4}}{\partial x_{5}} \\
\frac{\partial f_{5}}{\partial x_{1}} & \frac{\partial f_{5}}{\partial x_{2}} & \frac{\partial f_{5}}{\partial x_{3}} & \frac{\partial f_{5}}{\partial x_{4}} & \frac{\partial f_{5}}{\partial x_{5}}
\end{array}\right]
$$

Considering an initial value for each unknown, $x=x_{0}$, the next approximations are calculated by: 
$x_{n+1}=x_{n}-\Delta x_{n}$

where

$\Delta x_{n}=J^{-1} \cdot f\left(x_{n}\right)$

The solution continues until convergence occurs. Once all of the variables are calculated, the pressure is decreased by a fixed increment and the algorithm is repeated to find the new values. The algorithm continues to the pipe outlet, yielding all five unknowns along the pipe. Once the profiles of the velocities and volume fractions are obtained, they are used in the energy equation as follows:

$$
\begin{aligned}
& \left(\alpha_{g} \rho_{g} v_{g} C_{P g}+\alpha_{l} \rho_{l} v_{l} C_{P l}\right) \frac{\partial T}{\partial x}=\left(\alpha_{g} \rho_{g} v_{g} \eta_{g} C_{P g}+\alpha_{l} \rho_{l} v_{l} \eta_{l} C_{P l}\right) \frac{\partial P}{\partial x}+\alpha_{g} \rho_{g} v_{g} \frac{\partial}{\partial x}\left(\frac{P}{\rho_{g}}\right) \\
& +\alpha_{l} v_{l} \frac{\partial P}{\partial x}-P \frac{\partial\left(\alpha_{g} v_{g}+\alpha_{l} v_{l}\right)}{\partial x}+2 U \frac{r_{o}}{r_{i}^{2}}\left(T_{o}-T\right)
\end{aligned}
$$

The above equation is solved for $T$, the average temperature of the fluid mixture along the pipeline. Once the temperature profile is determined, it is compared with the initial assumption. If it is not close enough, the calculated temperature is used as the new assumption and all the steps are repeated until the residual between the two temperatures becomes sufficiently small.

\subsection{Three-phase flow solutions}

In the three-phase model, hydrate is assumed to form at the gas/liquid interface where both phases are present. Initially, the gas and liquid phases are present in the system with a constant mass flow rate. However, after the formation of hydrate crystals, the mass flow rate of the gas and liquid phases change along the pipeline.

Using the Newton-Raphson method, the nonlinear equations of continuity, momentum, and energy are solved simultaneously for velocity, volume fraction, pressure, and temperature. The continuity equations for a steady-state three phase flow are written as:

$\frac{d}{d x}\left(\alpha_{g} \rho_{g} v_{g}\right)=\Gamma_{h g}$ 
$\frac{d}{d x}\left(\alpha_{l} \rho_{l} v_{l}\right)=\Gamma_{h l}$

$\frac{d}{d x}\left(\alpha_{h} \rho_{h} v_{h}\right)=\Gamma_{g h}+\Gamma_{l h}$

where $\Gamma_{g h}$ and $\Gamma_{l h}$ represent the mass transfer rate from the gas and liquid phases to hydrate phase, respectively. Eqs. (96) to (98) can be written as:

$\alpha_{g} \rho_{g} v_{g} A_{g}=\dot{m}_{g}$

$\alpha_{l} \rho_{l} v_{l} A_{l}=\dot{m}_{l}$

$\alpha_{h} \rho_{h} v_{h} A_{h}=\dot{m}_{h}$

where unlike the two-phase model, the mass flow rates of the liquid $\left(\dot{m}_{l}\right)$ and gas $\left(\dot{m}_{g}\right)$ phases are changing due to the mass transfer from liquid and gas phases to hydrate phase. However, the total mass flow rate is constant.

The convective term in the momentum equation can be related to the continuity equation as follows:

$\frac{\partial\left(\alpha_{g} \rho_{g} v_{g}^{2}\right)}{\partial x}=v_{g} \frac{\partial}{\partial x}\left(\alpha_{g} \rho_{g} v_{g}\right)+\alpha_{g} \rho_{g} v_{g} \frac{\partial v_{g}}{\partial x}$

The first term on the right hand side of Eq. (102) can be simplified using Eq. (96), as follows:

$v_{g} \frac{\partial}{\partial x}\left(\alpha_{g} \rho_{g} v_{g}\right)=v_{g} \Gamma_{h g}$

Therefore, the convective term reduces to:

$\frac{\partial\left(\alpha_{g} \rho_{g} v_{g}^{2}\right)}{\partial x}=v_{g} \Gamma_{h g}+\alpha_{g} \rho_{g} v_{g} \frac{\partial v_{g}}{\partial x}$

And for liquid and hydrate phases:

$\frac{\partial\left(\alpha_{l} \rho_{l} v_{l}^{2}\right)}{\partial x}=v_{l} \Gamma_{h l}+\alpha_{l} \rho_{l} v_{l} \frac{\partial v_{l}}{\partial x}$

$\frac{\partial\left(\alpha_{h} \rho_{h} v_{h}^{2}\right)}{\partial x}=v_{h}\left(\Gamma_{g h}+\Gamma_{l h}\right)+\alpha_{h} \rho_{h} v_{h} \frac{\partial v_{h}}{\partial x}$

In the presented three-phase model, the governing equations are developed as follows:

$f_{1}(x)=\alpha_{g} \rho_{g} v_{g} A_{g}-\dot{m}_{g}=0$ 
$f_{2}(x)=\alpha_{l} \rho_{l} v_{l} A_{l}-\dot{m}_{l}=0$

$f_{3}(x)=\alpha_{h} \rho_{h} v_{h} A_{h}-\dot{m}_{h}=0$

$f_{4}(x)=\alpha_{g}+\alpha_{l}+\alpha_{h}-1=0$

$f_{5}(x)=v_{g} \Gamma_{h g}+\alpha_{g} \rho_{g} v_{g} \frac{\partial v_{g}}{\partial x}+\alpha_{g} \frac{d P}{d x}-F_{l g}-F_{w g}-v_{g} \Gamma_{h g}=0$

$f_{6}(x)=v_{l} \Gamma_{h l}+\alpha_{l} \rho_{l} v_{l} \frac{\partial v_{l}}{\partial x}+\alpha_{l} \frac{d P}{d x}-F_{g l}-F_{w l}-F_{h l}-v_{l} \Gamma_{h l}=0$

$f_{7}(x)=v_{h}\left(\Gamma_{g h}+\Gamma_{l h}\right)+\alpha_{h} \rho_{h} v_{h} \frac{\partial v_{h}}{\partial x}+\alpha_{h} \frac{d P}{d x}-F_{l h}-v_{h}\left(\Gamma_{g h}+\Gamma_{l h}\right)=0$

Eqs. (107) to (113) can be written as a single equation as follows:

$f(x)=0$

where $x$ represents a $7 \times 1$ matrix with 7 independent unknowns:

$x=\left[\begin{array}{l}x_{1} \\ x_{2} \\ x_{3} \\ x_{4} \\ x_{5} \\ x_{6} \\ x_{7}\end{array}\right]=\left[\begin{array}{l}\alpha_{g} \\ \alpha_{l} \\ \alpha_{h} \\ v_{g} \\ v_{l} \\ v_{h} \\ d P \\ d x\end{array}\right]$

And $f$ represents a $7 \times 1$ matrix containing 7 functions:

$f(x)=\left[\begin{array}{l}f_{1}(x) \\ f_{2}(x) \\ f_{3}(x) \\ f_{4}(x) \\ f_{5}(x) \\ f_{6}(x) \\ f_{7}(x)\end{array}\right]$

Based on the given equations and unknowns, the Jacobian matrix is calculated as follows: 


$$
J=\frac{\partial f}{\partial x}=\frac{\partial\left(f_{1}, f_{2}, f_{3}, f_{4}, f_{5}, f_{6}, f_{7}\right)}{\partial\left(x_{1}, x_{2}, x_{3}, x_{4}, x_{5}, x_{6}, x_{7}\right)}=\left[\begin{array}{lllllll}
\frac{\partial f_{1}}{\partial x_{1}} & \frac{\partial f_{1}}{\partial x_{2}} & \frac{\partial f_{1}}{\partial x_{3}} & \frac{\partial f_{1}}{\partial x_{4}} & \frac{\partial f_{1}}{\partial x_{5}} & \frac{\partial f_{1}}{\partial x_{6}} & \frac{\partial f_{1}}{\partial x_{7}} \\
\frac{\partial f_{2}}{\partial x_{1}} & \frac{\partial f_{2}}{\partial x_{2}} & \frac{\partial f_{2}}{\partial x_{3}} & \frac{\partial f_{2}}{\partial x_{4}} & \frac{\partial f_{2}}{\partial x_{5}} & \frac{\partial f_{2}}{\partial x_{6}} & \frac{\partial f_{2}}{\partial x_{7}} \\
\frac{\partial f_{3}}{\partial x_{1}} & \frac{\partial f_{3}}{\partial x_{2}} & \frac{\partial f_{3}}{\partial x_{3}} & \frac{\partial f_{3}}{\partial x_{4}} & \frac{\partial f_{3}}{\partial x_{5}} & \frac{\partial f_{3}}{\partial x_{6}} & \frac{\partial f_{3}}{\partial x_{7}} \\
\frac{\partial f_{4}}{\partial x_{1}} & \frac{\partial f_{4}}{\partial x_{2}} & \frac{\partial f_{4}}{\partial x_{3}} & \frac{\partial f_{4}}{\partial x_{4}} & \frac{\partial f_{4}}{\partial x_{5}} & \frac{\partial f_{4}}{\partial x_{6}} & \frac{\partial f_{4}}{\partial x_{7}} \\
\frac{\partial f_{5}}{\partial x_{11}} & \frac{\partial f_{5}}{\partial x_{2}} & \frac{\partial f_{5}}{\partial x_{3}} & \frac{\partial f_{5}}{\partial x_{4}} & \frac{\partial f_{5}}{\partial x_{5}} & \frac{\partial f_{5}}{\partial x_{6}} & \frac{\partial f_{5}}{\partial x_{7}} \\
\frac{\partial f_{6}}{\partial x_{1}} & \frac{\partial f_{6}}{\partial x_{2}} & \frac{\partial f_{6}}{\partial x_{3}} & \frac{\partial f_{6}}{\partial x_{4}} & \frac{\partial f_{6}}{\partial x_{5}} & \frac{\partial f_{6}}{\partial x_{6}} & \frac{\partial f_{6}}{\partial x_{7}} \\
\frac{\partial f_{7}}{\partial x_{1}} & \frac{\partial f_{7}}{\partial x_{2}} & \frac{\partial f_{7}}{\partial x_{3}} & \frac{\partial f_{7}}{\partial x_{4}} & \frac{\partial f_{7}}{\partial x_{5}} & \frac{\partial f_{7}}{\partial x_{6}} & \frac{\partial f_{7}}{\partial x_{7}}
\end{array}\right]
$$

To initiate the solution, a value $\left(x=x_{0}\right)$ is assumed for each variable, and the next approximations are calculated as follows:

$x_{n+1}=x_{n}-\Delta x_{n}$

where

$\Delta x_{n}=J^{-1} \cdot f\left(x_{n}\right)$

The solution continues until the set convergence occurs. Once all of the seven variables are found, the pressure is decreased by a fixed increment and the algorithm is repeated to find the new values in the next block. A $5 \mathrm{~m}$ discrete grid spacing $(\Delta x)$ is considered from the pipe inlet to the pipe outlet. The algorithm continues to the pipe outlet, yielding all seven unknowns along the pipe. Once the velocity and volume fraction distributions of the phases are calculated, they are used in the energy equation as follows:

$$
\begin{aligned}
& \left(\alpha_{g} \rho_{g} v_{g} C_{P g}+\alpha_{l} \rho_{l} v_{l} C_{P l}+\alpha_{h} \rho_{h} v_{h} C_{P h}\right) \frac{\partial T}{\partial x}=\left(\alpha_{g} \rho_{g} v_{g} \eta_{g} C_{P g}+\alpha_{l} \rho_{l} v_{l} \eta_{l} C_{P l}+\alpha_{h} \rho_{h} v_{h} \eta_{h} C_{P h}\right) \frac{\partial P}{\partial x} \\
& +\alpha_{g} \rho_{g} v_{g} \frac{\partial}{\partial x}\left(\frac{P}{\rho_{g}}\right)+\alpha_{l} v_{l} \frac{\partial P}{\partial x}+\alpha_{h} v_{h} \frac{\partial P}{\partial x}-P \frac{\partial}{\partial x}\left(\alpha_{g} v_{g}+\alpha_{l} v_{l}+\alpha_{h} v_{h}\right)+2 U \frac{r_{o}}{r_{i}^{2}}\left(T_{o}-T\right)
\end{aligned}
$$

The above equation is solved for the average temperature of the mixture fluid along the pipeline. Once the average temperature profile is determined, it is compared with the initial assumption. The iteration is performed until the residual between the two consecutive temperatures becomes very small. 


\section{RESULTS AND DISCUSSION}

\subsection{Parameters and properties}

Consider multiphase flow in an uninsulated pipeline with natural gas (consisting of mostly methane), water, and hydrate particles. Problem parameters and thermophysical properties of the fluids and gas are summarized in the table below.

Table 1. Problem parameters and properties

\begin{tabular}{|c|c|}
\hline Parameter & Value \\
\hline Water density & $995.7 \mathrm{~kg} / \mathrm{m}^{3}$ \\
\hline Inner pipe diameter & $0.408 \mathrm{~m}$ \\
\hline Outer pipe diameter & $0.415 \mathrm{~m}$ \\
\hline Gas dynamic viscosity & 0.0000106 Pa.s \\
\hline Water dynamic viscosity & $0.000821 \mathrm{~Pa} . \mathrm{s}$ \\
\hline Gas constant & $518.3 \mathrm{~J} /(\mathrm{kg} . \mathrm{K})$ \\
\hline Inlet pressure & $10 \mathrm{MPa}$ \\
\hline Total mass flow rate & $17.2 \mathrm{~kg} / \mathrm{s}$ \\
\hline Gas mass flow rate & $1.2 \mathrm{~kg} / \mathrm{s}$ \\
\hline Liquid mass flow rate & $16 \mathrm{~kg} / \mathrm{s}$ \\
\hline Inlet temperature & $333 \mathrm{~K}$ \\
\hline Surrounding water temperature & $280 \mathrm{~K}$ \\
\hline Water heat capacity & $4,184 \mathrm{~J} / \mathrm{K}$ \\
\hline Gas heat capacity & $2,226 \mathrm{~J} / \mathrm{K}$ \\
\hline Pipe wall thermal conductivity & $54 \mathrm{~W} /(\mathrm{m} . \mathrm{K})$ \\
\hline Gas thermal conductivity & $0.026 \mathrm{~W} /(\mathrm{m} . \mathrm{K})$ \\
\hline Water thermal conductivity & $0.615 \mathrm{~W} /(\mathrm{m} . \mathrm{K})$ \\
\hline Gas Joule-thomson coefficient & $0.000002 \mathrm{~K} / \mathrm{Pa}$ \\
\hline
\end{tabular}




\section{$5.2 \quad$ Two-phase models}

In order to analyze the steady-state two-phase flow problem, a 30,000 m subsea pipeline with a $40.8 \mathrm{~cm}$ inner diameter and $7 \mathrm{~mm}$ wall thickness is considered. The flow pattern of gas-liquid flow is assumed to be a stratified flow. Inlet boundary conditions of 333 $\mathrm{K}$ for temperature and $10 \mathrm{MPa}$ for pressure were considered. An inlet mass flow rate of 16 $\mathrm{kg} / \mathrm{s}$ for liquid and $1.2 \mathrm{~kg} / \mathrm{s}$ for gas flow was considered.

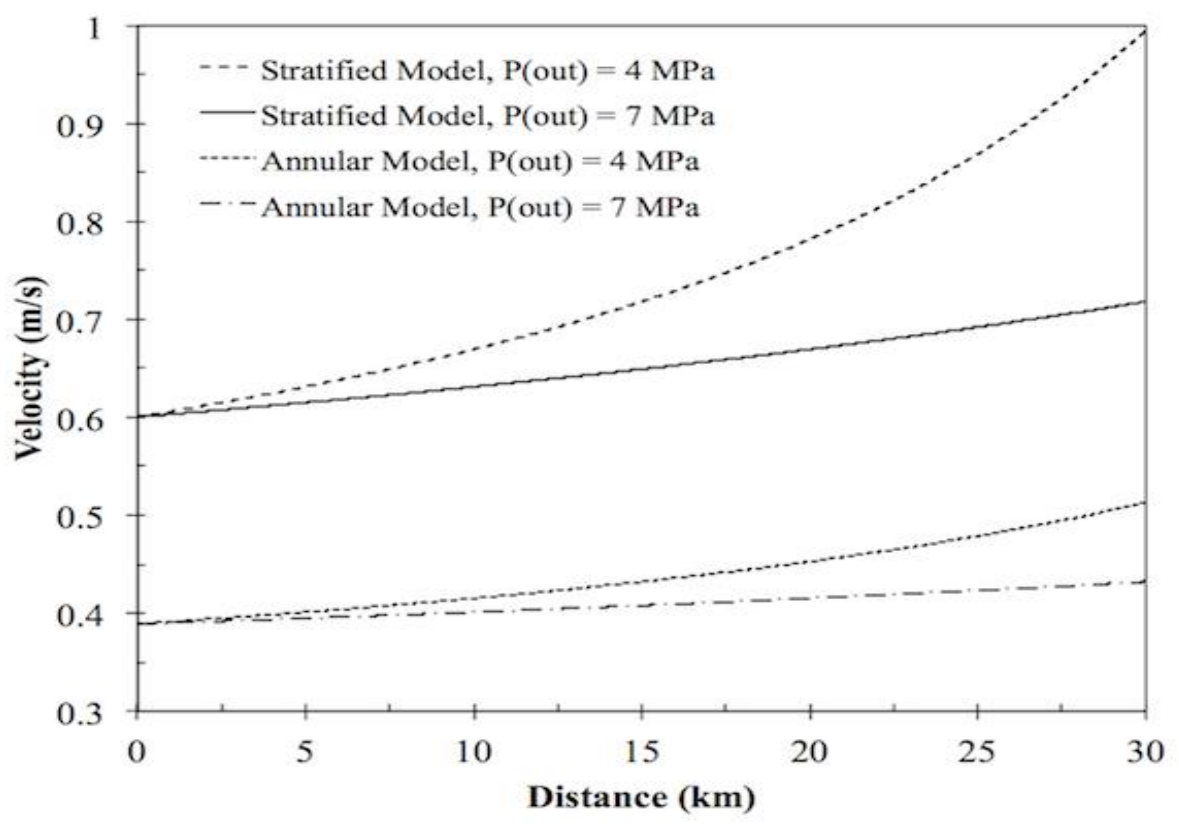

Figure 11. Distributions of steady-state velocity along the pipeline for stratified and annular models for different outlet pressures

Figure 11 illustrates the predicted steady-state behaviour of fluid velocity along the pipe. The mixture velocity increases along the pipe as shown in Figure 11. This trend is expected as the velocity of each phase increases along the pipe. The gas density decreases due to the pressure drop, and this decline is offset by the increase of velocity to satisfy the continuity equation. The velocity profile is affected by varying the outlet pressure. At a lower outlet pressure, the fluid velocity increases considerably towards the outlet. Figure 11 reveals that the velocity rise in the stratified model is higher than the annular model. For the liquid phase, shown in Figure 12, the volume fraction decreases by the increase in the gas velocity, 
causing the liquid velocity to increase. This decrease in the liquid volume fraction results in a slight rise in the gas volume fraction (Figure 13) to satisfy the volume fraction balance equation $\left(\alpha_{g}+\alpha_{l}=1\right)$.

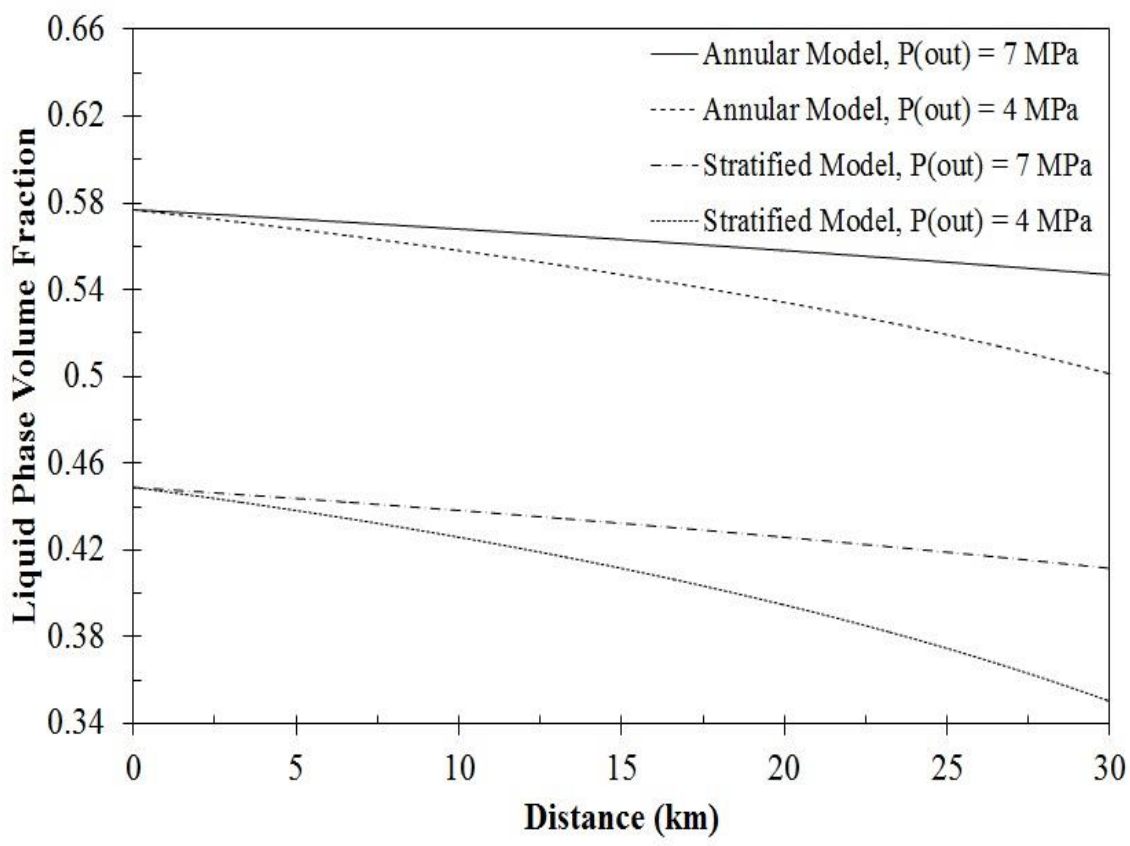

Figure 12. Changes in liquid volume fraction along the pipeline for stratified and annular models for different outlet pressures

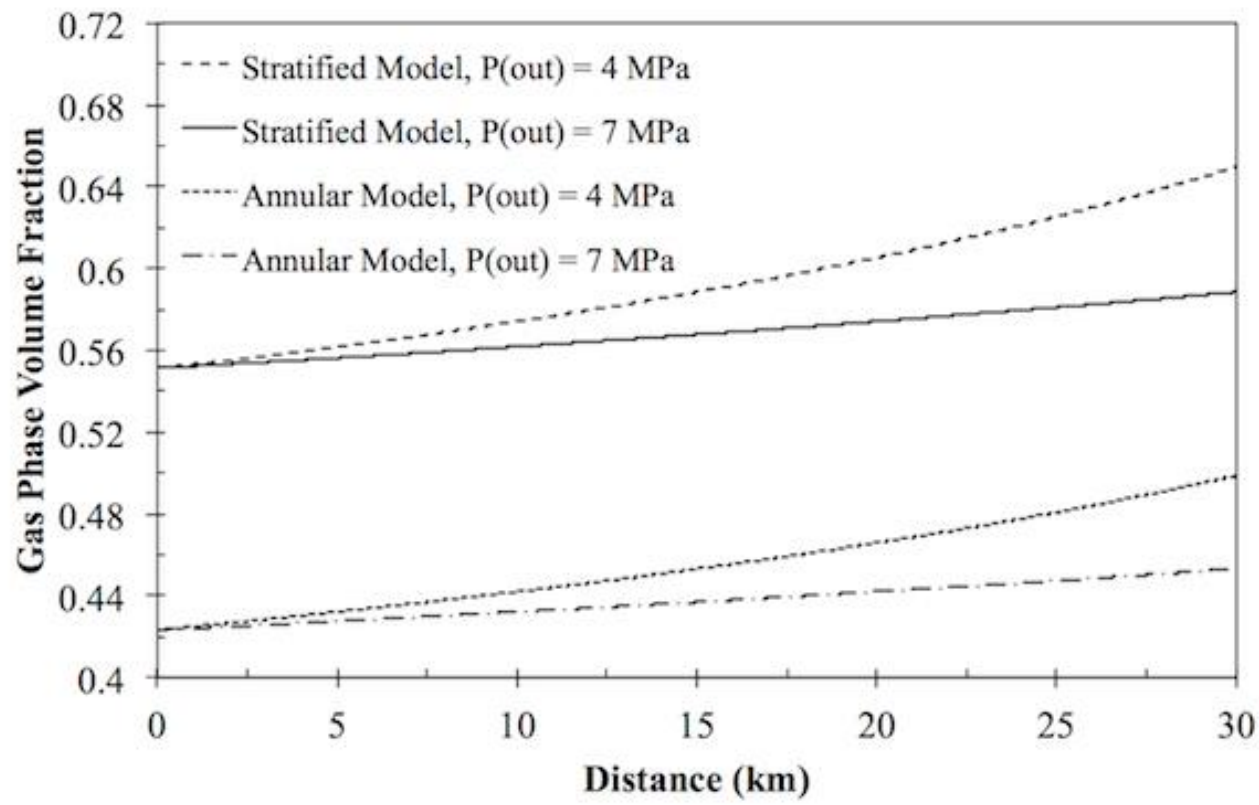

Figure 13. Changes in Gas volume fraction along the pipeline for stratified and annular models for different outlet pressures 
Figure 14 shows changes in the gas density along the pipeline. The gas expands significantly due to the pressure drop towards the pipe's outlet. As expected, a higher pressure drop causes the gas density to decline more in this particular case.

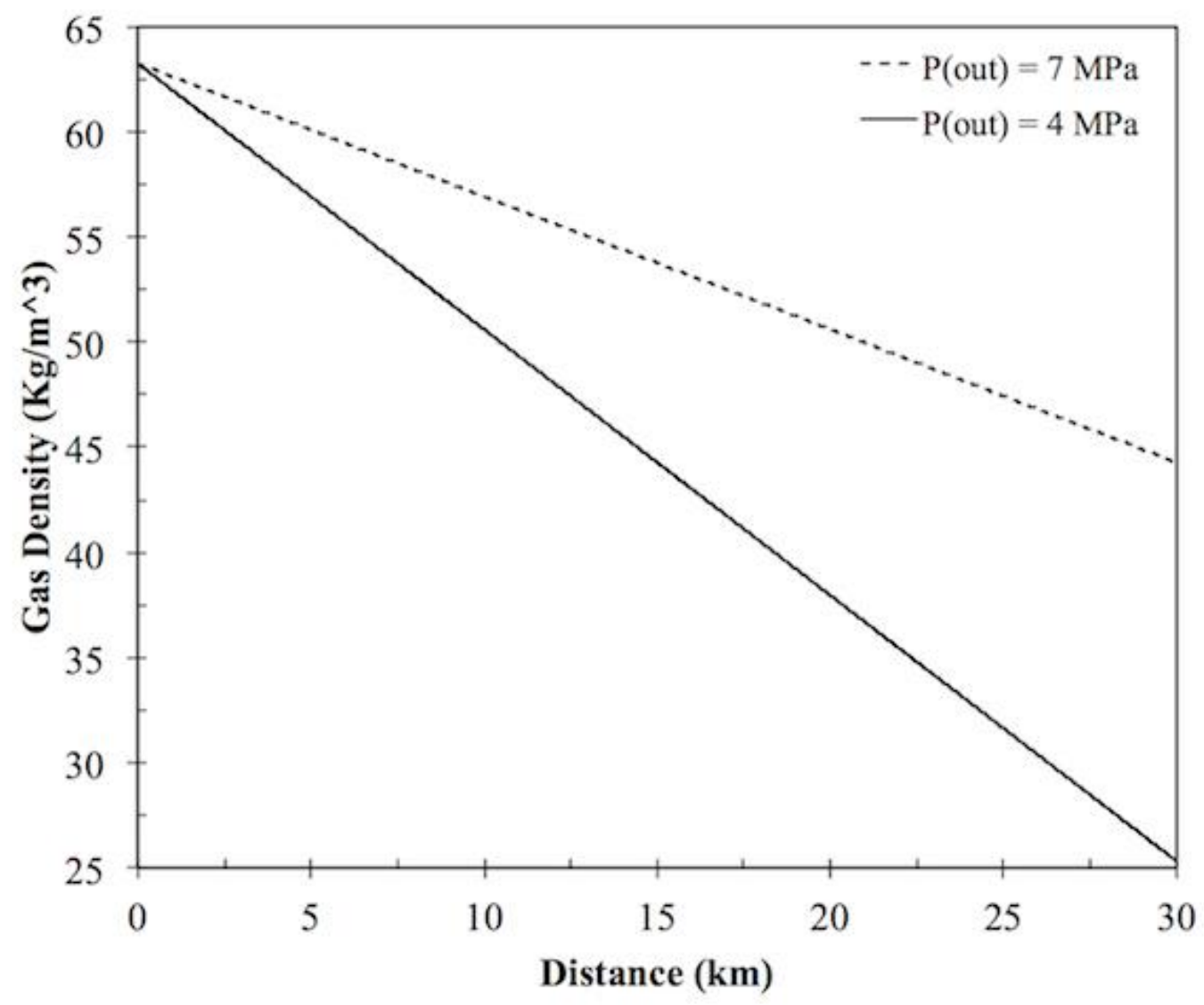

Figure 14. Gas density distribution along the pipeline for different outlet pressures

The pressure drop, as seen in Figure 15, follows a nearly linear trend from $10 \mathrm{MPa}$ at the inlet to $4 \mathrm{MPa}$ and $7 \mathrm{MPa}$ at the outlet. The drop in pressure is due to wall friction. Comparing Figure 11 and Figure 15 reveals that higher velocities lead to a larger pressure drop. 


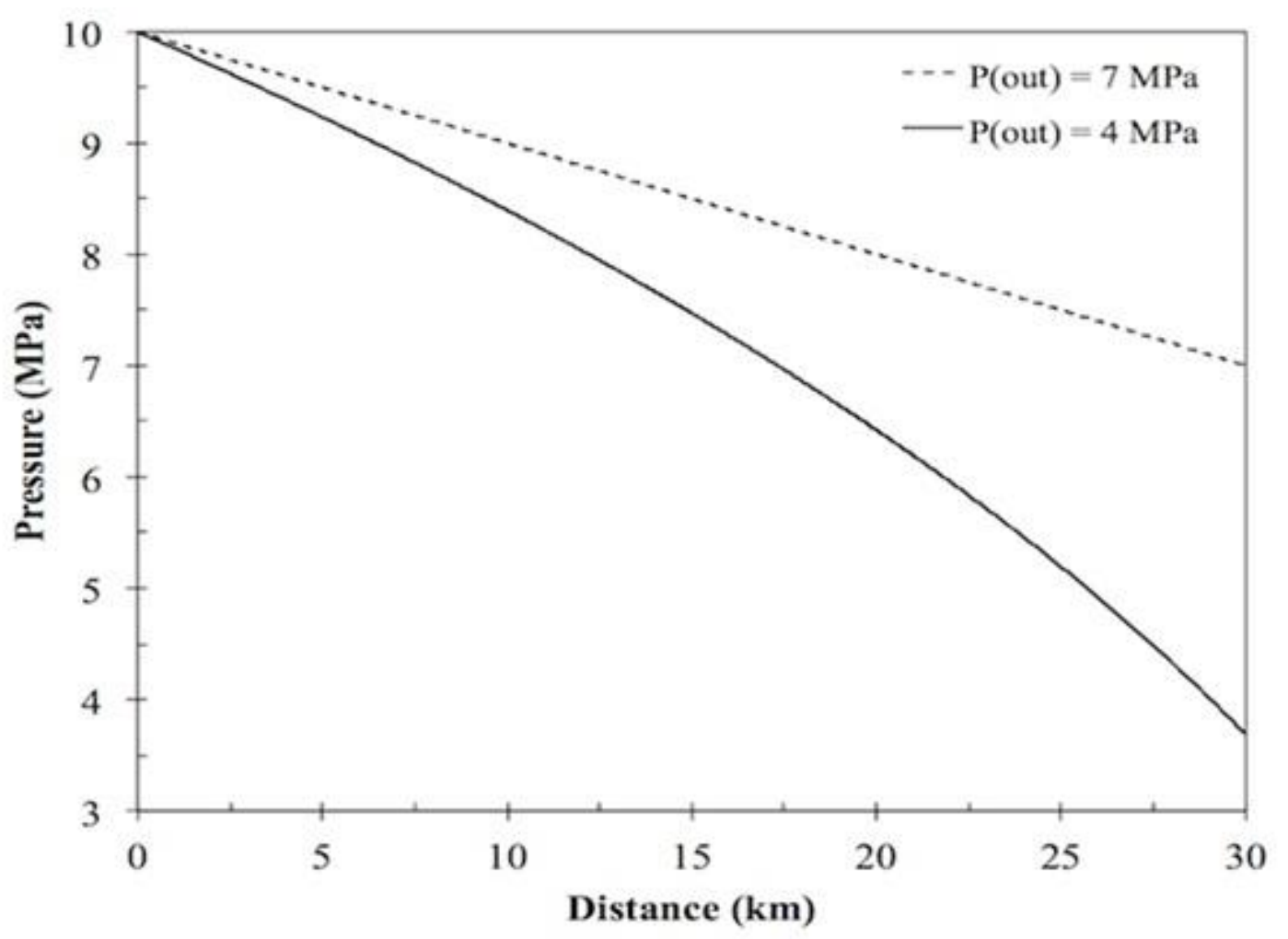

Figure 15. Pressure profiles along the pipeline

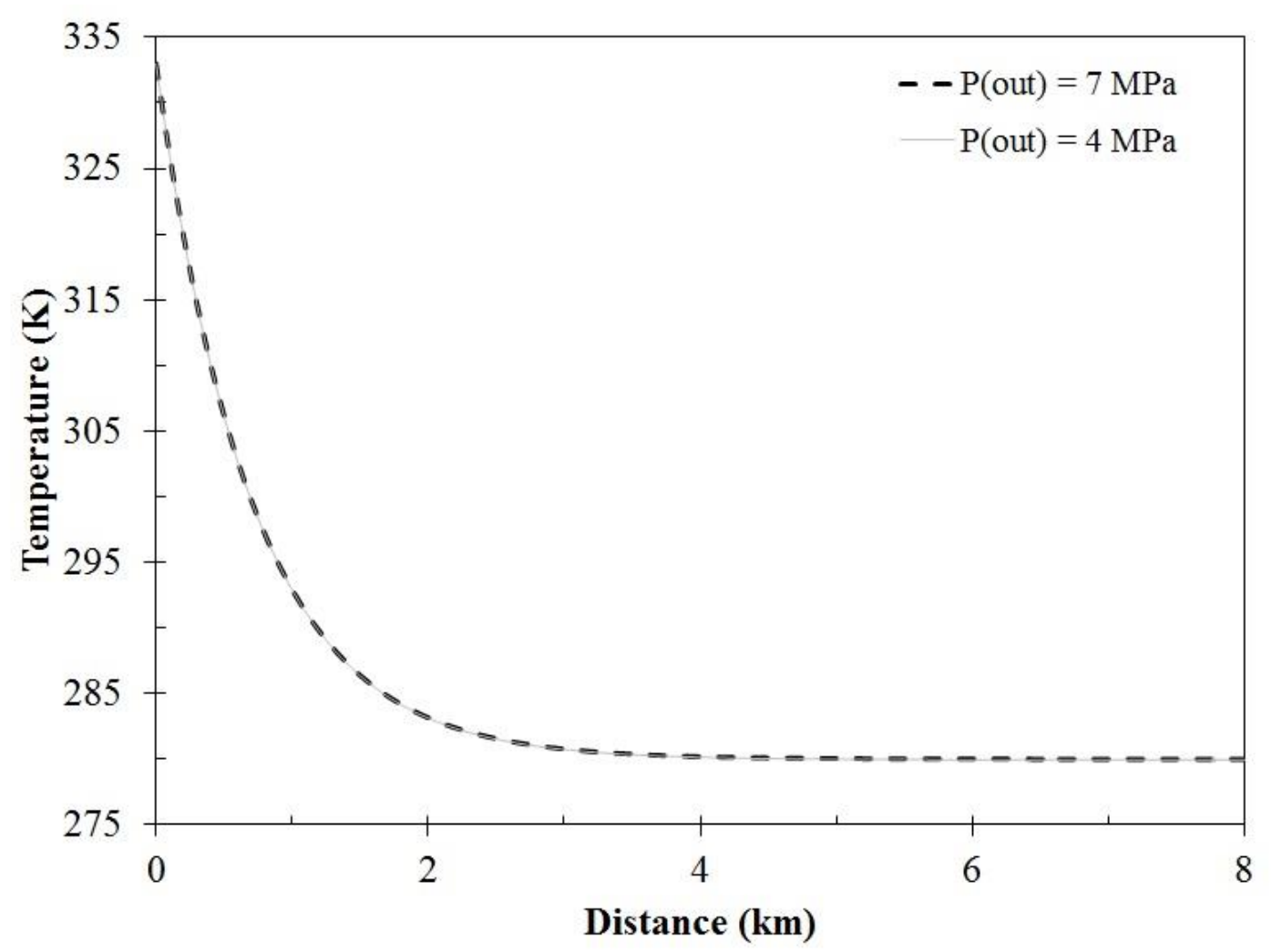

Figure 16. Temperature profiles for different outlet pressures

The temperature profile, shown in Figure 16, reveals that the fluid temperature drops 
significantly near the pipe inlet and gradually approaches the surrounding temperature. The sharp temperature drop is due to the large Nusselt number, indicating that convection from the outer surface is dominant which causes the flow to cool rapidly until it approaches the seawater temperature. This occurs where there is potential for hydrate particles to form and grow, as the fluid temperature is low, and the pressure is still high (Jung et al., 2012). A varying outlet pressure does not affect the temperature profile significantly, as shown in Figure 16. Referring to Figures 15 and 16 reveals that hydrates are more likely to form and grow when there is a lower pressure drop. In this case, the fluid pressure is higher, and the temperature is low, which is a favorable condition for hydrates to grow.

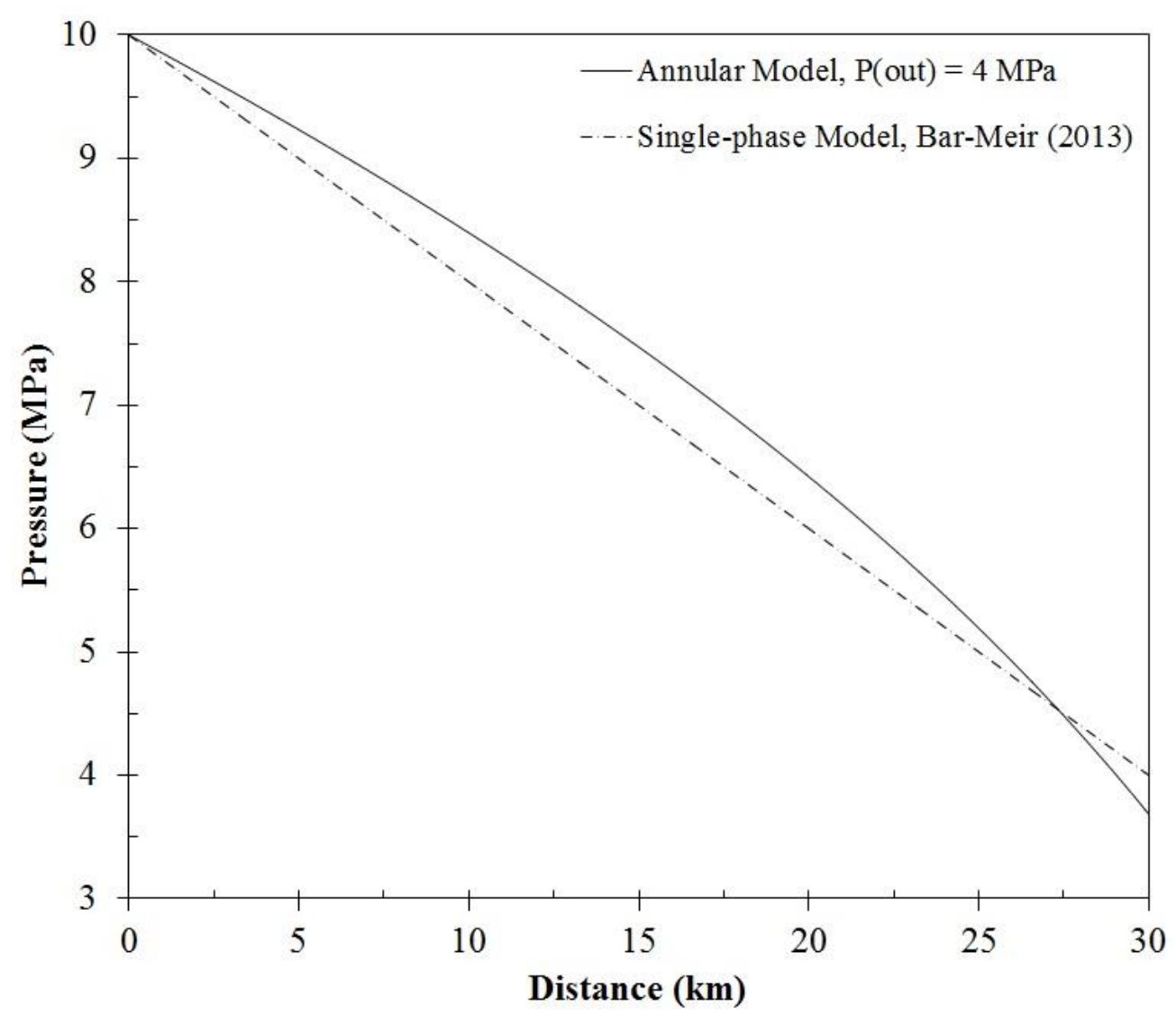

Figure 17. Validation of pressure profile for two-phase annular model against a single-phase model (Bar-Meir, 2013) with mixture properties

Figs. 17 and 18 show validation studies of the pressure and temperature profiles. The results are compared against a single-phase model containing a mixture of natural gas and 
water. Based on the single-phase models presented by Bar-Meir (2013) and Bergman et al. (2011), pressure and temperature drop have been calculated using mixture properties. The results of these single-phase models (shown in Figs. 17 and 18) are found to be in relatively good agreement with the current two-phase models. A discrepancy in Figures 17 and 18 is expected due to no interaction between phases in the single-phase model. The two-phase model accounts for friction between phases, and predicts the flow characteristics more accurately.

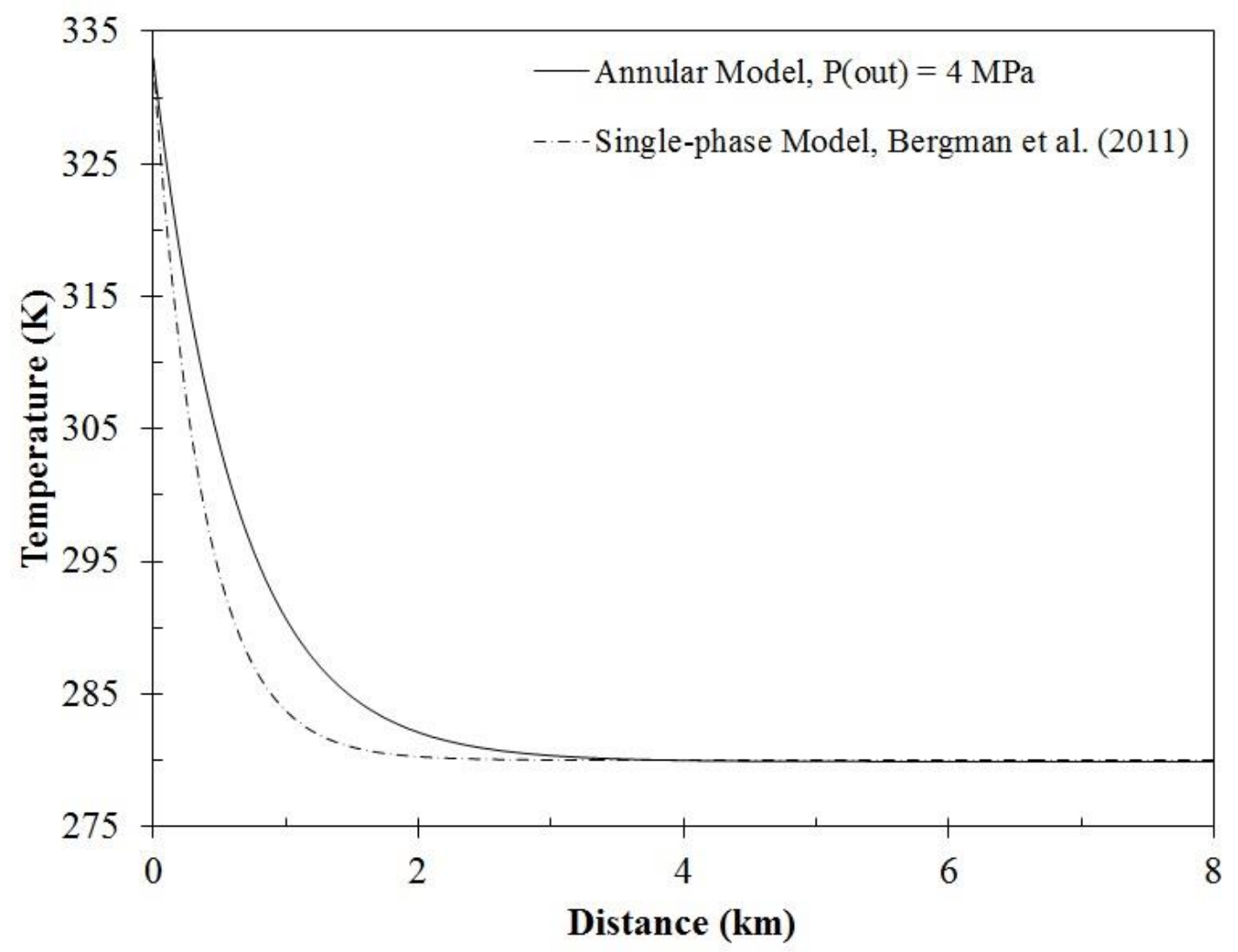

Figure 18. Validation of temperature profile for two-phase annular model against a single-phase model (Bergman et al., 2011) with mixture properties

The results for the gas/hydrate model are presented in Figures 19, 20 and 21. Figure 19 confirms that the velocity profile for the gas/hydrate model follows the same trend as the velocity in the gas/water models. The fluid velocity is higher in comparison to the previous models. In a similar way, the gas phase volume fraction increases for both outlet pressures. 
However, the hydrate volume fraction follows a slight drop. The velocity rise of the hydrate phase causes the solid particles to gradually occupy a greater portion of the pipe. This leads to a decrease in the volume fraction of hydrate along the pipeline to conserve mass.

The fluid temperature, as seen in Figure 21, drops quickly to the pipe wall temperature and remains unchanged towards the pipe outlet. This occurs due to the large Nusselt number, meaning that convection between the fluid and pipe wall is relatively high. The flow cools to the pipe wall temperature $(280 \mathrm{~K})$ and remains at this temperature. The low temperature and high pressure after this point provide a favorable environment for hydrates to form and grow. The results for all models confirm that outlet pressure does not affect the temperature profile, but can change fluid velocity significantly.

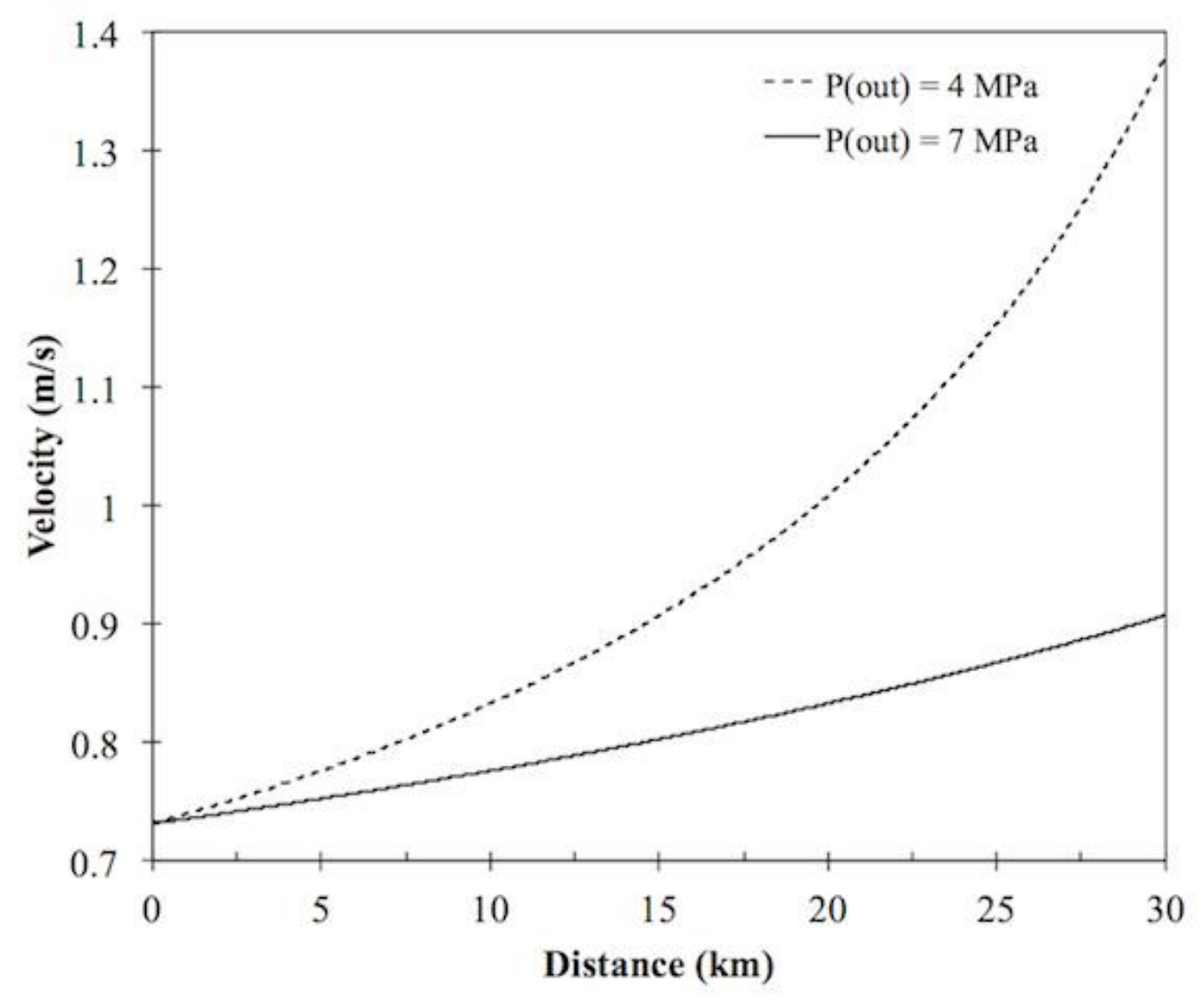

Figure 19. Distributions of steady-state velocity along the pipeline for gas/hydrate annular model for different outlet pressures 


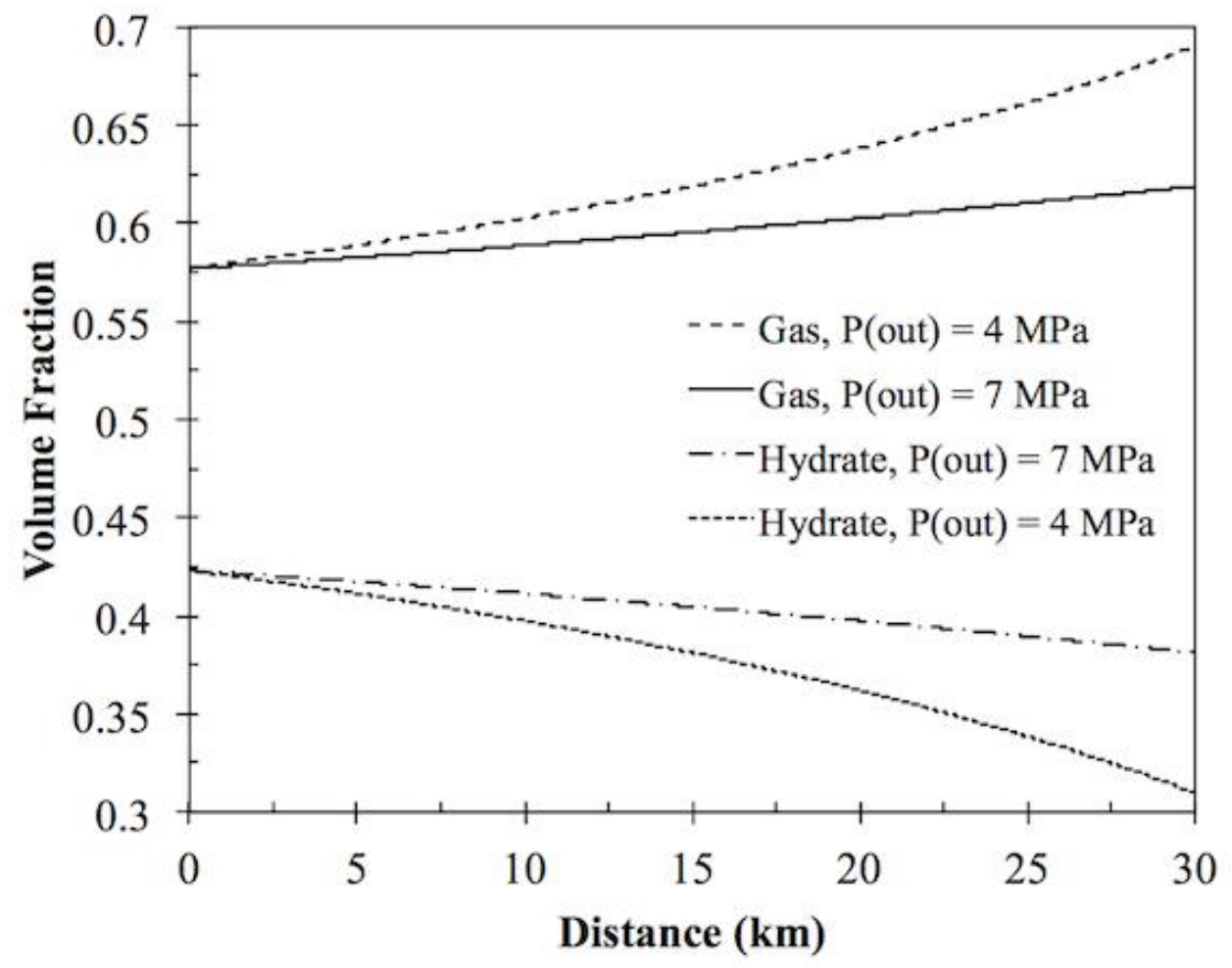

Figure 20. Volume fraction profiles along the pipeline for gas/hydrate model for different outlet pressures

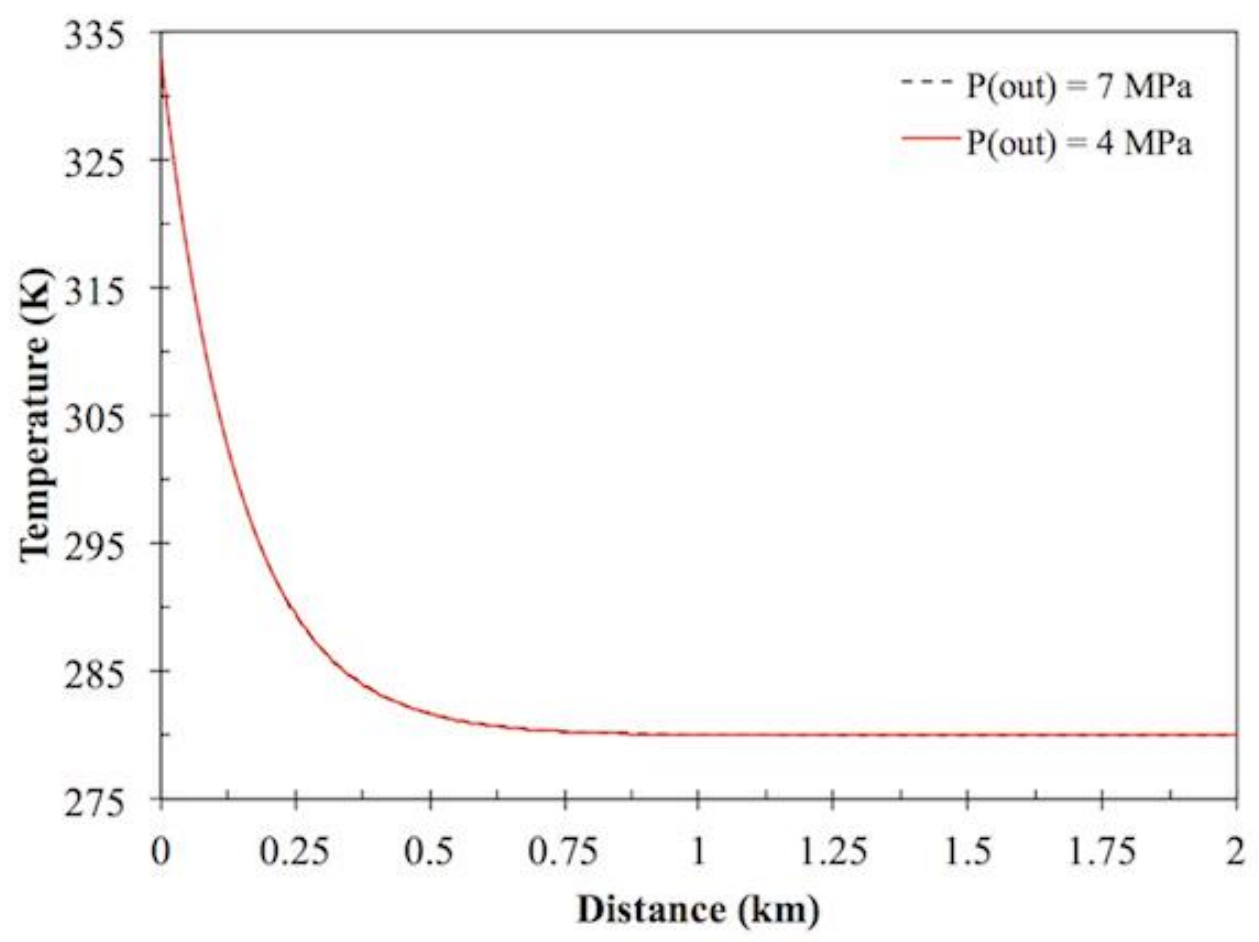

Figure 21. Temperature distribution along the pipeline for gas/hydrate model for different outlet pressures 


\subsection{Three-phase model}

A 7,000 m subsea pipeline with a $40.8 \mathrm{~cm}$ inner diameter and $7 \mathrm{~mm}$ thickness is considered. The three-phase flow pattern of gas/liquid/hydrates flow is assumed to be a stratified flow. Inlet boundary conditions of $333 \mathrm{~K}$ for temperature and $10 \mathrm{MPa}$ for pressure were considered. An inlet mass flow rate of $16 \mathrm{~kg} / \mathrm{s}$ for liquid and $1.2 \mathrm{~kg} / \mathrm{s}$ for gas was considered. Based on the above assumptions, the nonlinear systems of equations were solved.

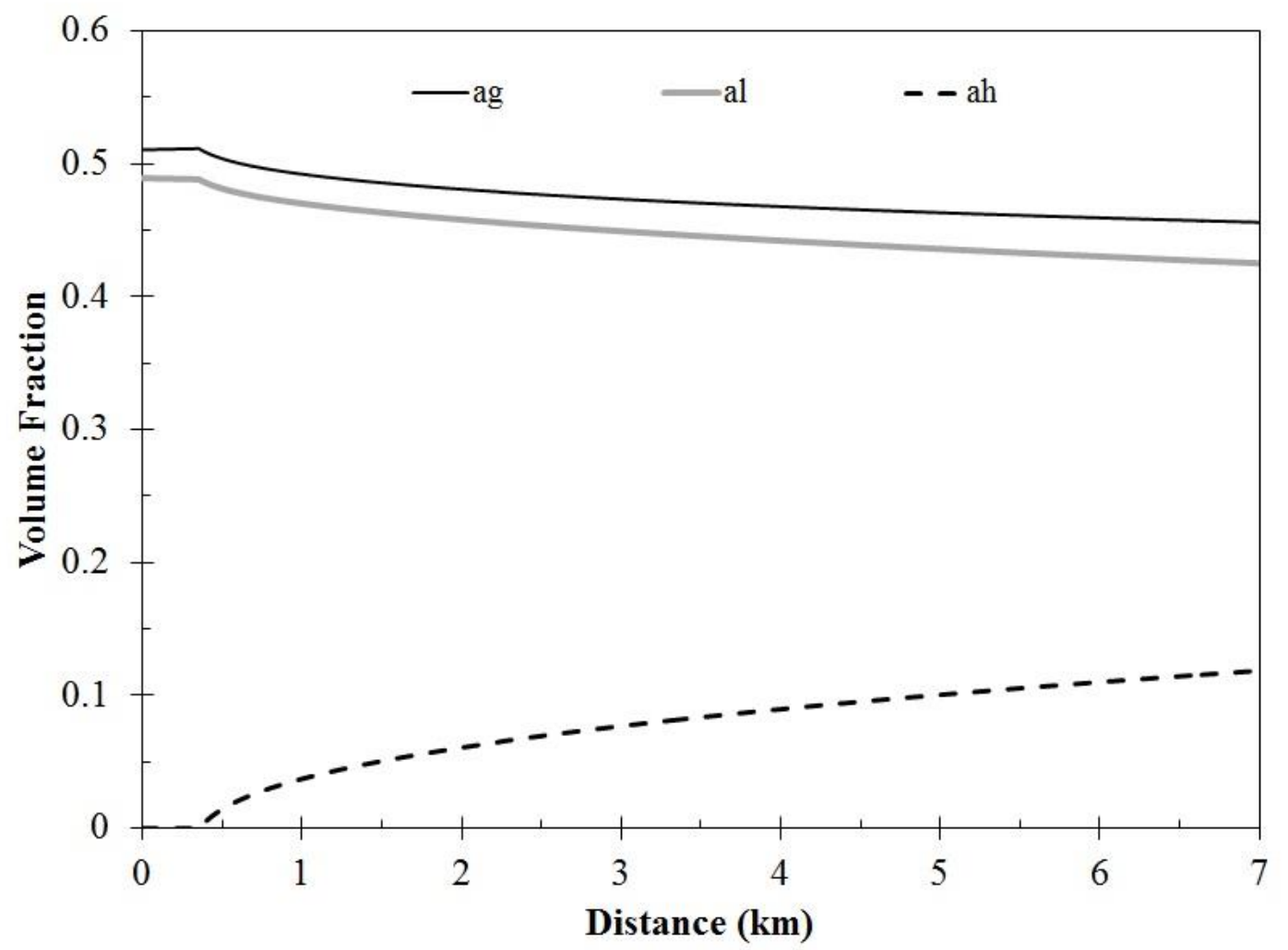

Figure 22. Volume fraction of the phases along the pipeline length

The volume fraction of gas and liquid are initially constant as there is no hydrate formation. They remain constant until the equilibrium conditions are met and the first hydrate crystal is formed. Then, liquid and gas volume fractions decrease while the hydrate phase is growing. The sum of volume fractions for the three phases adds to one. It is seen in Figure 22 that early in the pipeline, the rate of hydrate growth is higher. This result is expected as the pressure and temperature conditions are more favorable for hydrate growth. The pressure and temperature are initially high before the formation stage. Then, when the temperature drops 
to the equilibrium temperature, hydrates are formed and they grow quickly because the pressure is still high. However, the pressure gradually decreases and slows the rate of hydrate formation. The results in Figure 22 reveal that after $7 \mathrm{~km}$, almost 12 percent of the pipeline volume is occupied by the hydrate phase, if no inhibitor is used to prevent the hydrate formation.

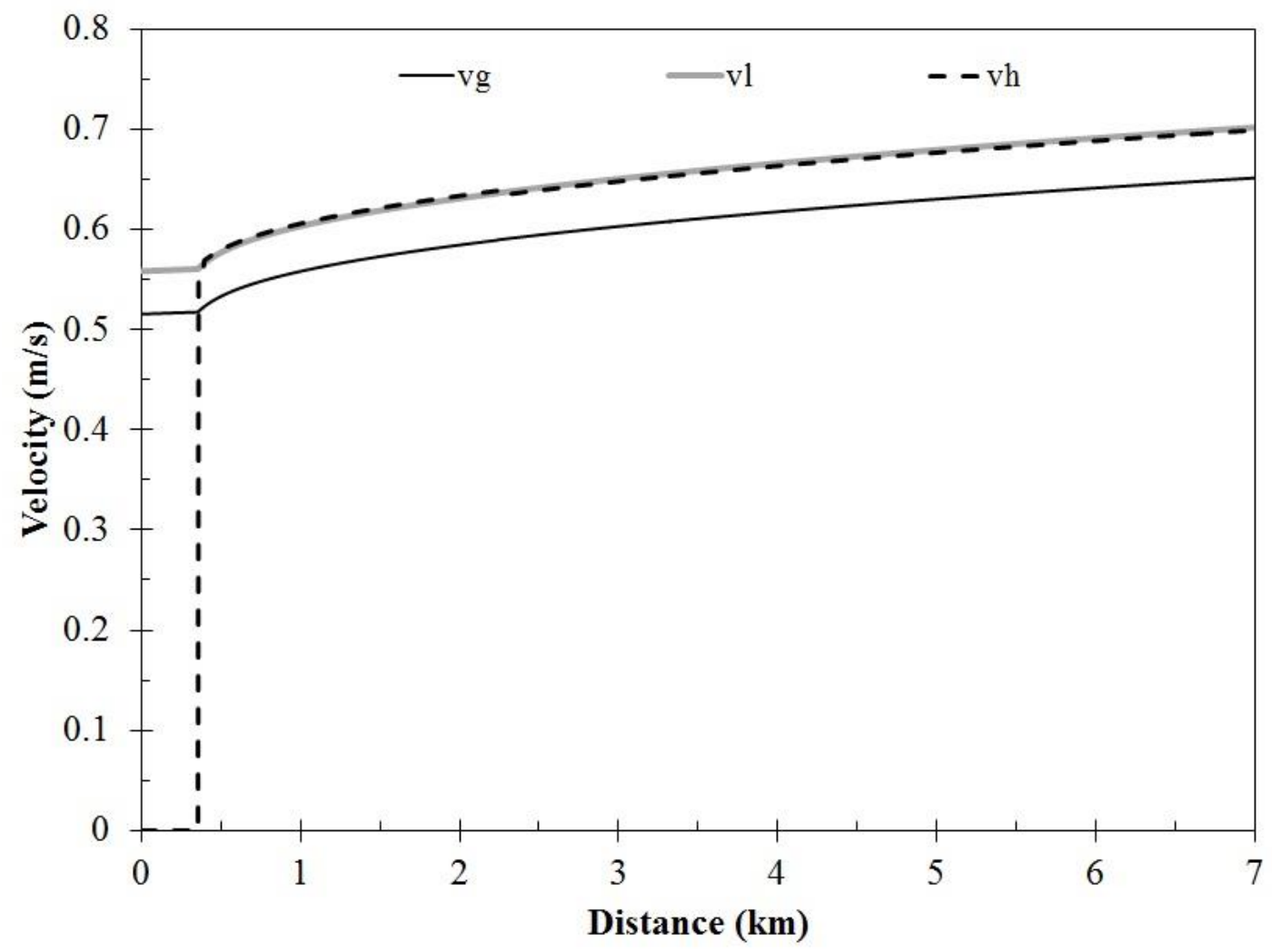

Figure 23. Velocity of the phases along the pipeline length

Liquid and gas velocities, as seen in Figure 23, remain constant until the flow reaches the equilibrium conditions. From there onwards, the velocities of all three phases increase due to the change in gas density resulting from the pressure drop. Figure 23 reveals that liquid and hydrate phases have almost the same velocity, which is expected based on the assumption that hydrate particles are carried by the liquid phase. The results from Figure 23 confirm that the stratified model is a realistic model based on the liquid and gas velocities. Based on Figure 2, the stratified model is applicable for low gas and liquid velocities (less than $0.1 \mathrm{~m} / \mathrm{s}$ ), which is the case in this study. 
Figure 24 shows the pressure drop along the pipeline. As illustrated in Fig. 24, the pressure drop follows a nearly linear trend, which is a driving mechanism for the change in other variables like velocities and volume fractions. Refering to Figure 5, it can be seen that the pressure distribution is still high causing a potential condition for hydrate formation. Considering the pressure profile and the temperature profile (Figure 25), it is revealed that they both imply conditions for the formation of hydrate particles (Figure 5).

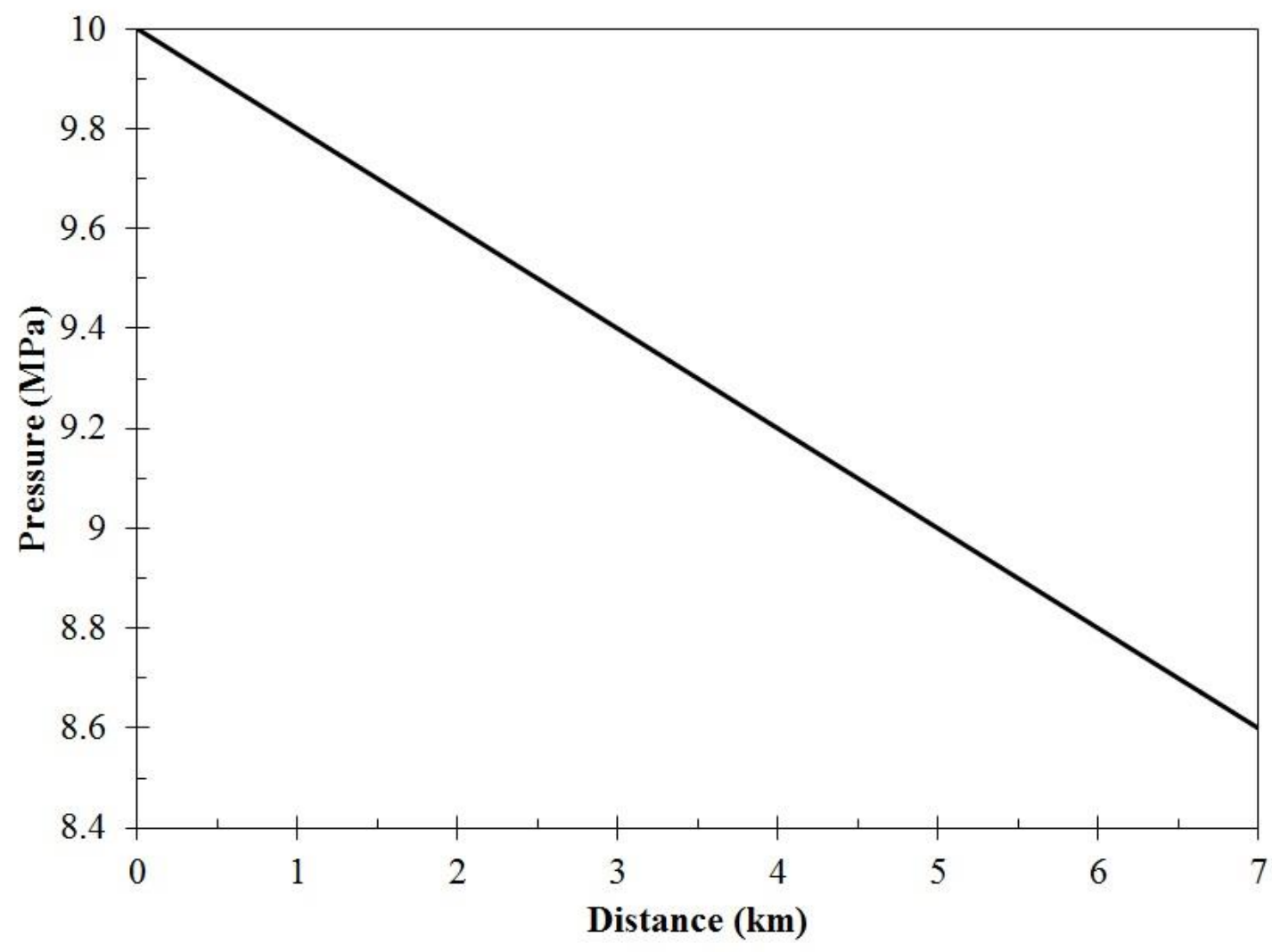

Figure 24. Pressure distribution for the fluid mixture

The temperature distribution confirms that initially the rate of temperature drop is high. Therefore, the fluid temperature enters the hydrate forming region where hydrates may be formed. The temperature remains below the equilibrium temperature until the pipe outlet. The hydrates grow after the equilibrium condition as long as the pressure is higher than the equilibrium pressure (Figure 5). 


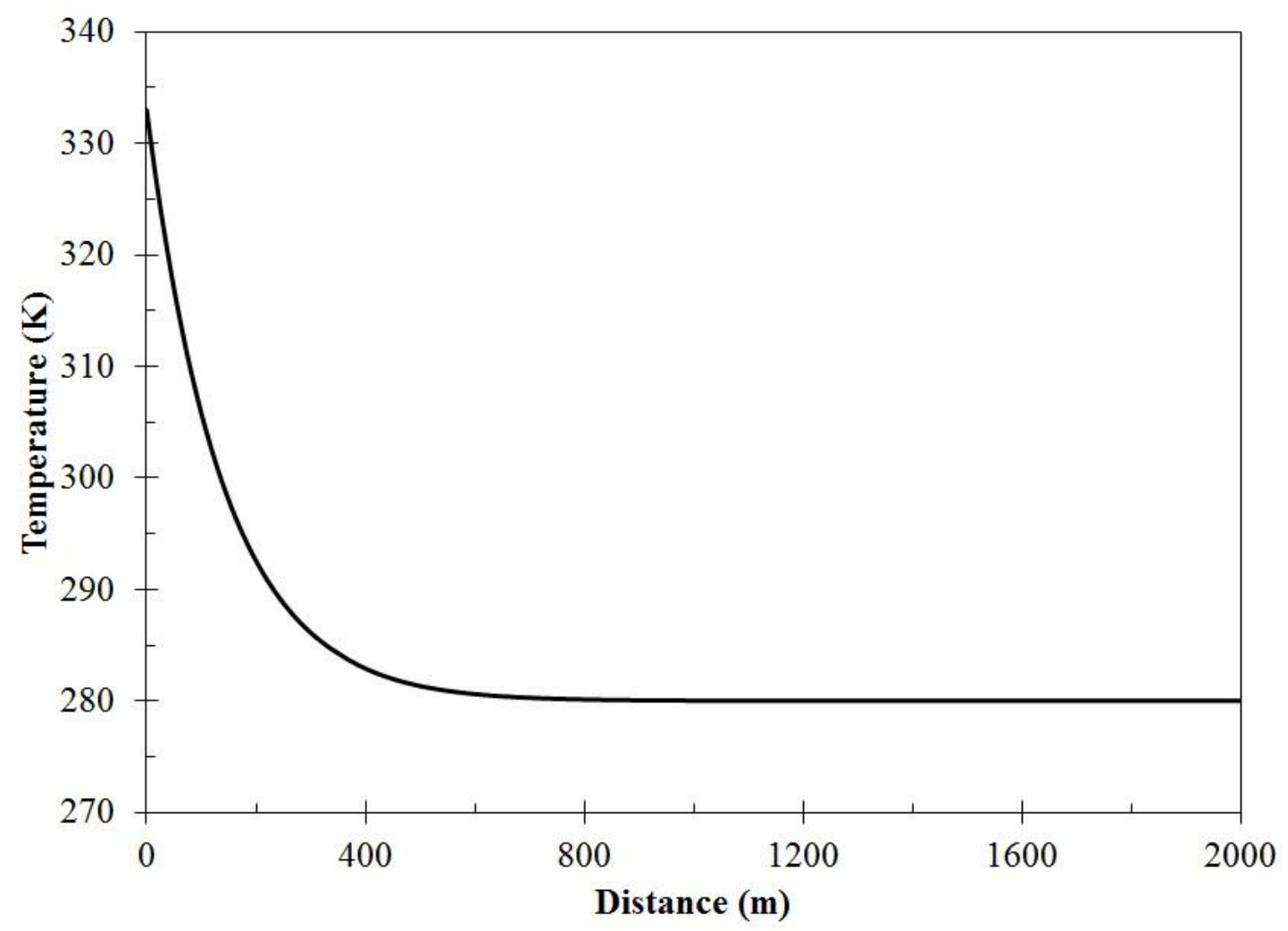

Figure 25. Temperature distribution for the fluid mixture

\subsection{Parametric studies}

To assess the impact of varying parameters on the flow characteristics, parametric studies have been conducted. In this section, the effects of variations in the pipe diameter, mass flow rate, inlet pressure, and inlet temperature are studied, and the results are presented and discussed.

In the first set of graphs (Figs. 26 to 30), the effect of a change in the pipe diameter is shown. It is expected that increasing the diameter results in later formation of hydrate particles. With an increase in the diameter, the pipe surface area is higher, meaning more heat is transferred from the mixture fluid. However, conduction heat transfer in the mixture is reduced due to the higher film thickness of each phase. This can decrease the overall heat transfer coefficient (Eq. 54) and cause the mixture to cool slower, resulting in a higher temperature distribution. A higher temperature distribution causes the fluid to reach the equilibrium conditions at a later stage along the pipeline and finally to later formation of 
hydrate crystals. In Fig. 26, hydrates in a pipe with a larger diameter start to form later than smaller pipes. Figure 26 also reveals that a higher proportion of the pipe is occupied by the hydrate phase due to the increase in the diameter. The hydrate and gas phase volume fractions are expected to have opposite trends as a part of the gas phase is consumed by the hydrate phase. Therefore, the drop in the volume fraction of the gas phase increases when the pipe diameter is increased.

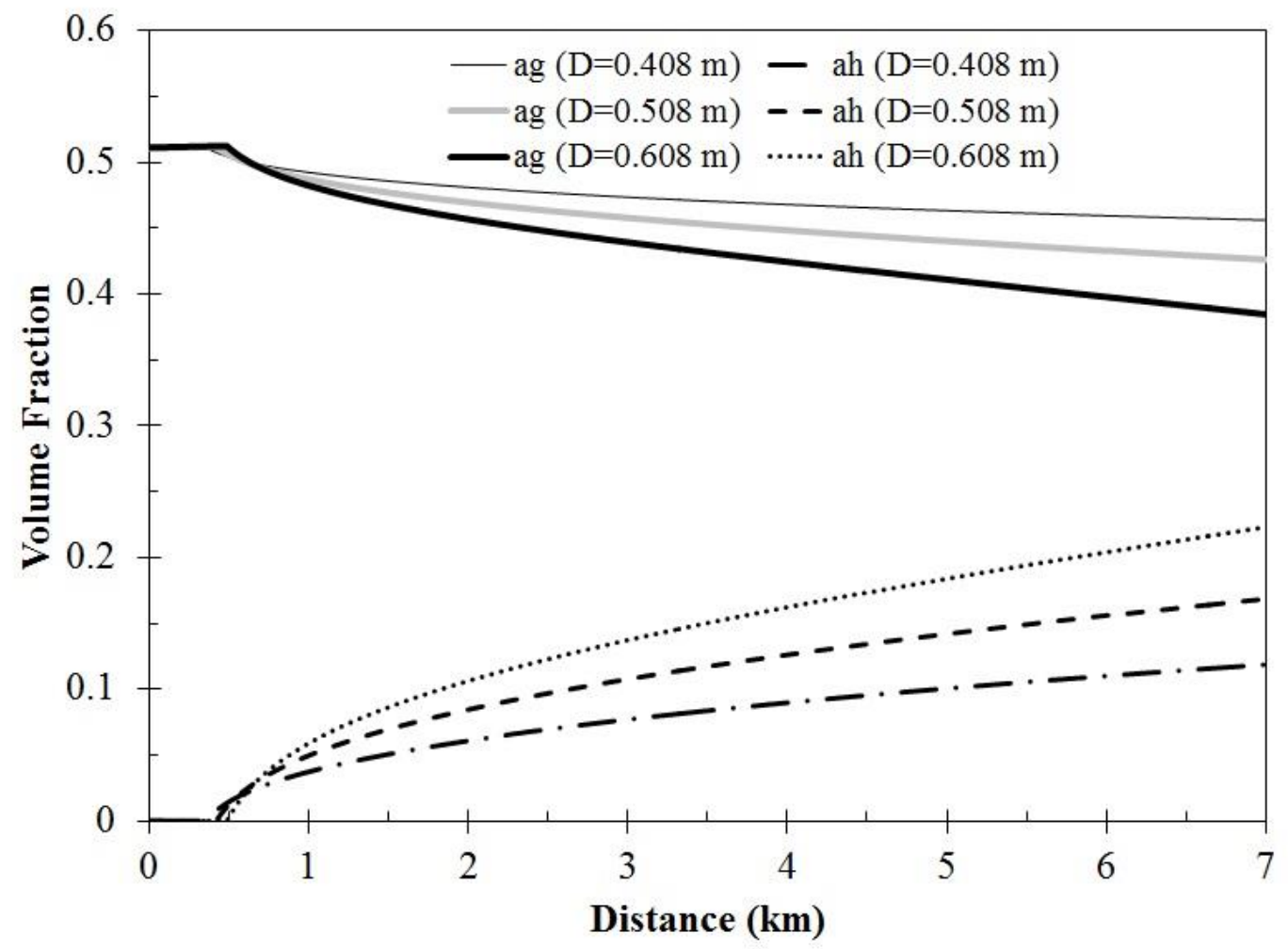

Figure 26. Volume fraction distribution of the gas and hydrate along the pipeline for different pipe diameters $(\mathrm{ag}=$ gas volume fraction, $\mathrm{ah}=$ hydrate volume fraction, $\mathrm{D}=$ diameter $)$

The liquid phase volume fraction has the same trend as the gas phase. As shown in Figure 27, for a pipe with a larger diameter, a higher volume fraction drop is seen for the liquid phase. Comparing Figures 26 and 27 reveals that the sum of volume fractions is equal to one. 


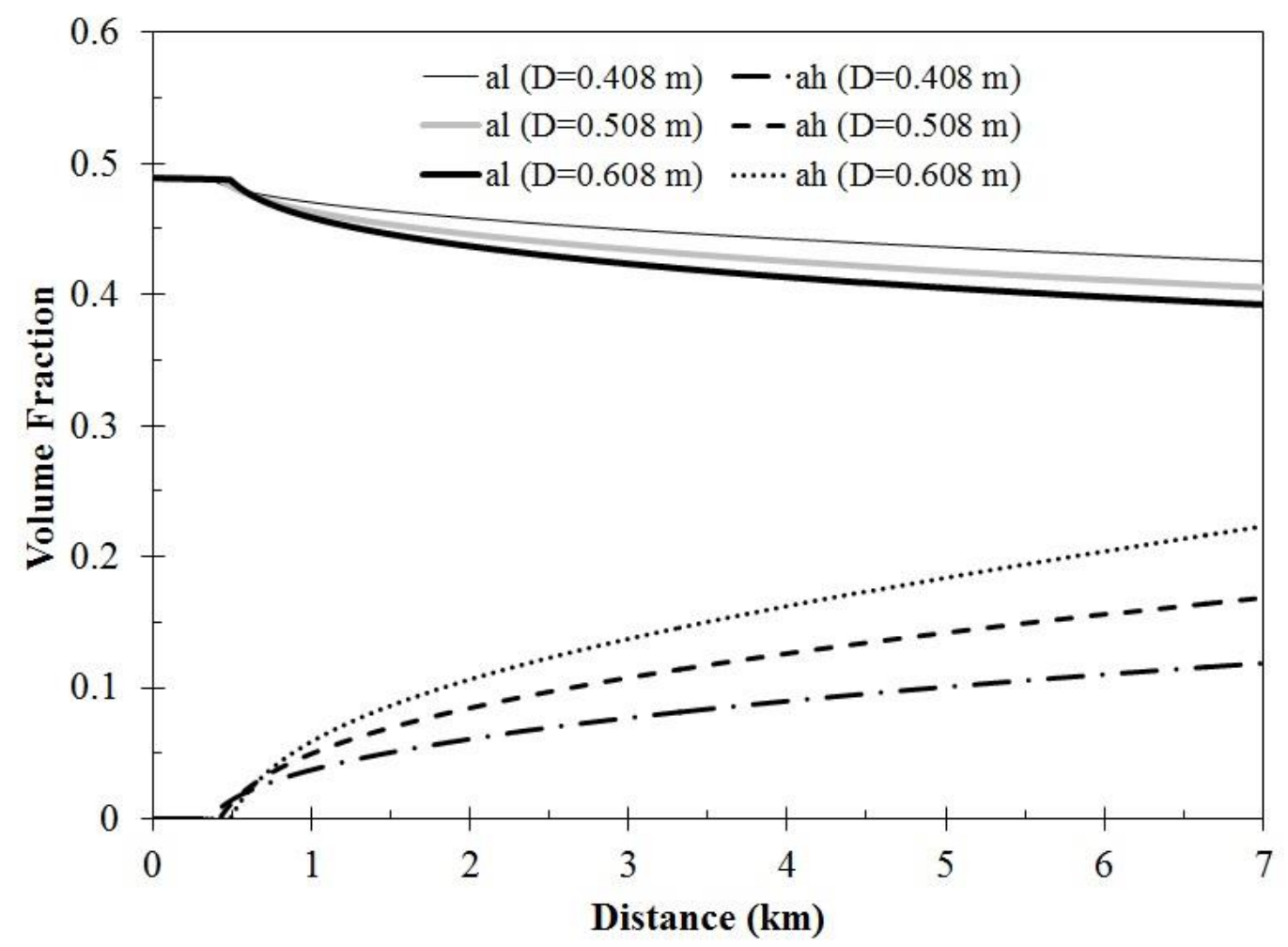

Figure 27. Volume fraction distribution of the liquid and hydrate along the pipeline for different pipe diameters $(\mathrm{al}=$ gas volume fraction, $\mathrm{ah}=$ hydrate volume fraction, $\mathrm{D}=$ diameter $)$

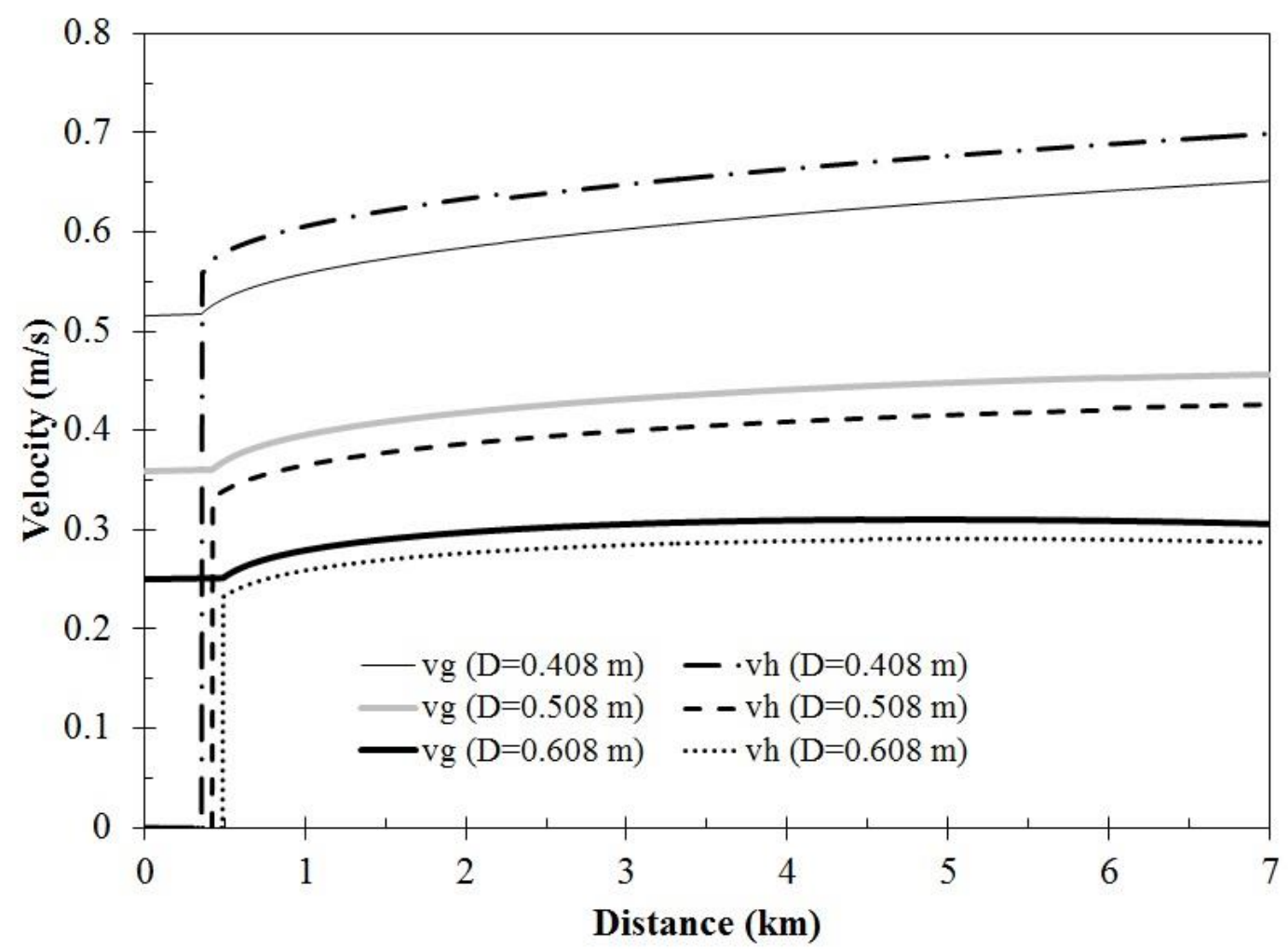

Figure 28. Velocity distribution of the gas and hydrate along the pipeline for different pipe diameters 


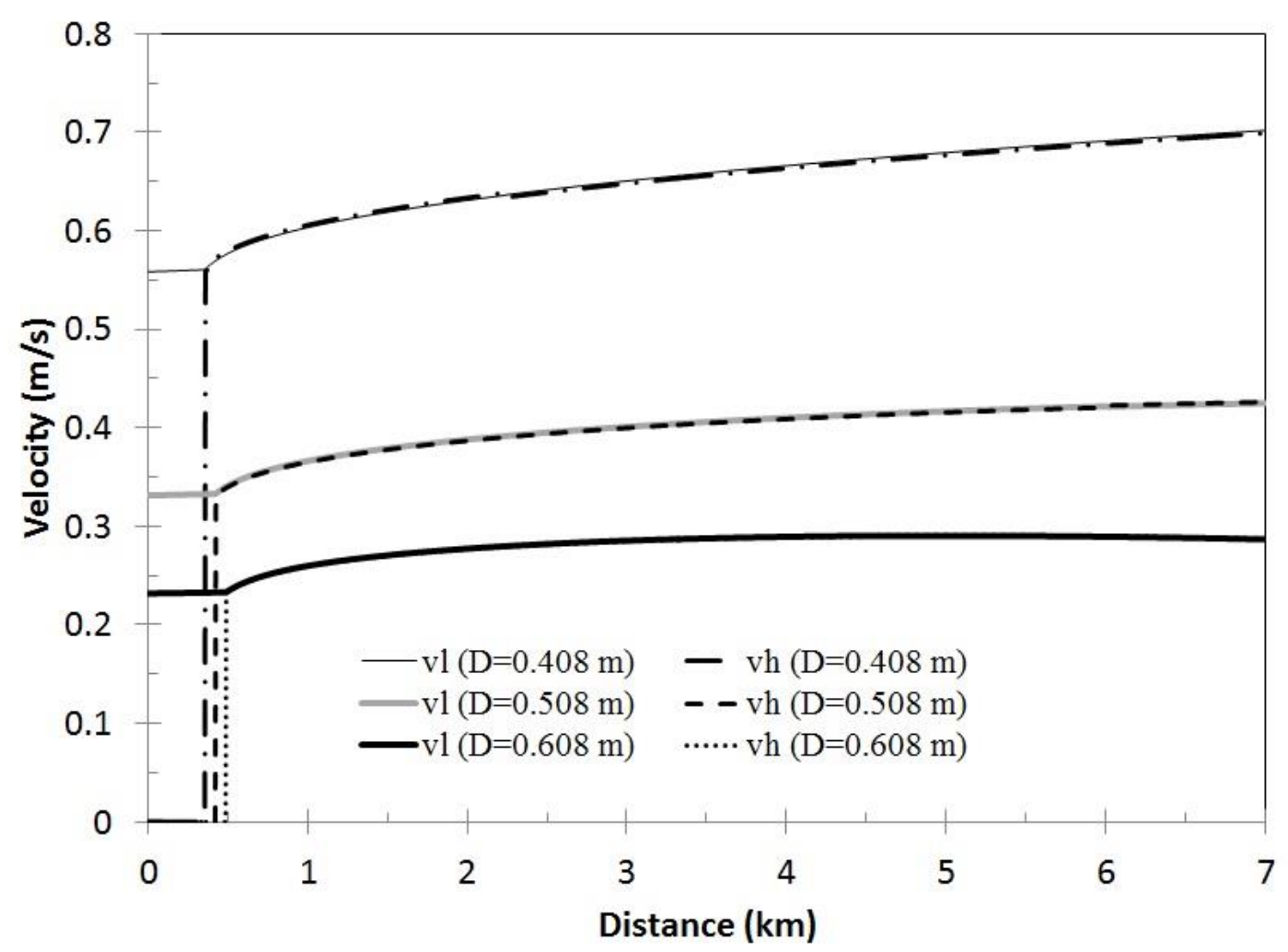

Figure 29. Velocity distribution of the liquid and hydrate along the pipeline for different pipe diameters

Figs. 28 and 29 show the velocities of the phases along the pipeline. Eqs. 107, 108, and 109 suggest that for a constant mass flow rate, if the pipe diameter (or cross-sectional area) increases, the velocity decreases to balance the continuity equation. Figs. 28 and 29 confirm this and show that the velocity of the phases drops when the diameter of the pipe increases. It is also observed that as a result of a sudden change in the system due to the hydrate formation, the rate of change in the mixture velocity is higher after the point where the hydrates are formed. Then the velocity gradient is gradually reduced towards the pipeline outlet.

Figure 30 shows the system temperature along the pipeline length for three different pipe diameters. As explained previously, due to the increase of the diameter, the overall heat transfer coefficient $(\mathrm{U})$ is lower, causing the fluid temperature to drop less. The effect of variations of pipe diameter on the system temperature is not significant. Increasing the pipe 
diameter postpones the formation of hydrate crystals, but the effect on temperature distribution is only noticeable in the pipe entry region. After some distance from the pipe inlet, the mixture fluid temperature gradually drops to the surrounding temperature.

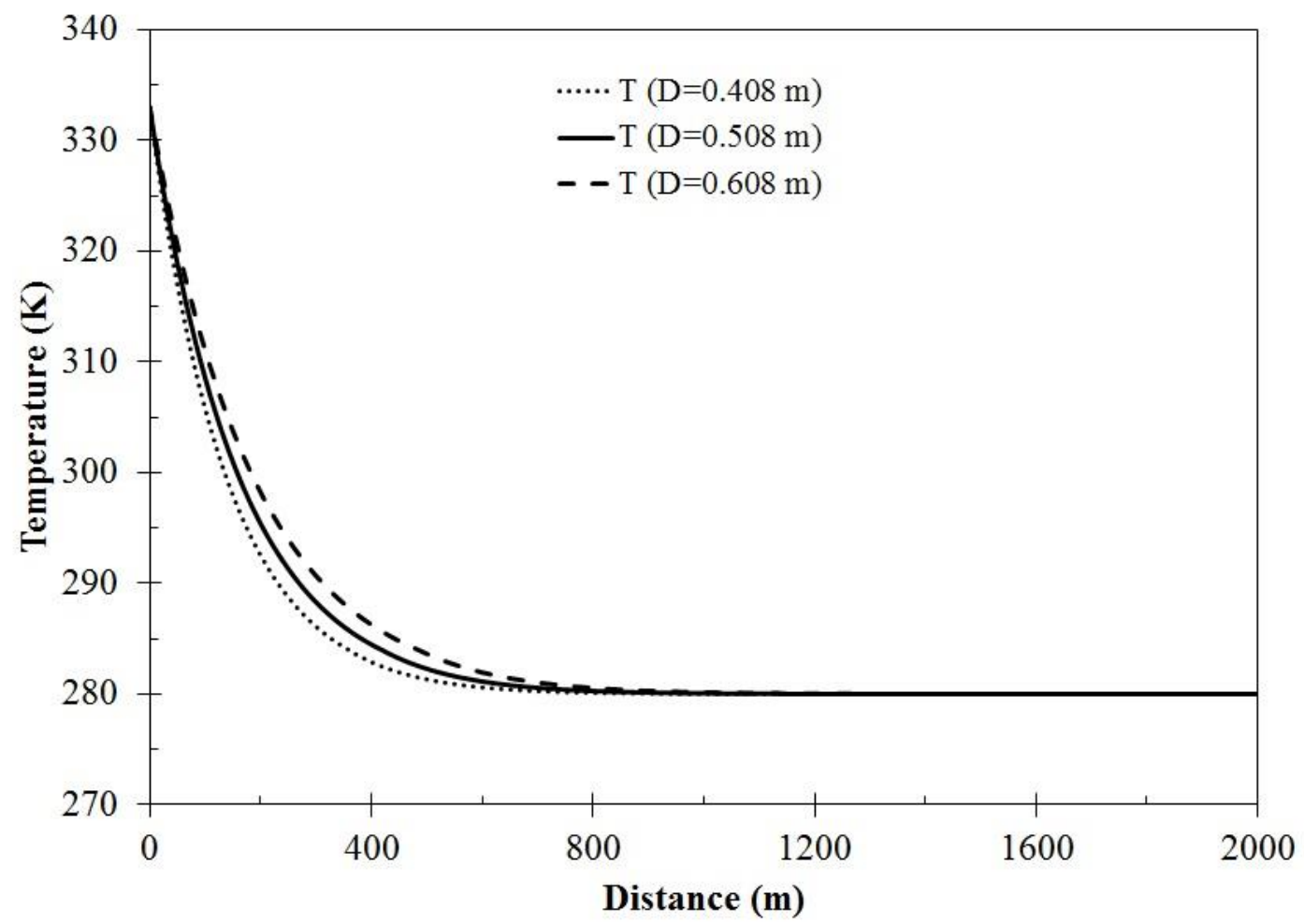

Figure 30. Temperature distribution of the mixture fluid along the pipeline for different pipe diameters

The results of the parametric studies also reveal the effects of variations of the mass flow rate on the flow characteristics (Figures 31 to 34). If the mass flow rate of the gas phase increases, the gas phase volume fraction is expected to increase as well. This is confirmed by Fig. 31, which shows an increasing trend for the volume fraction when the flow rate for the gas phase is higher. With regard to the hydrate phase, the formation stage is altered by a variation of the mass flow rates. Increasing the liquid mass flow rate reduces the temperature, meaning hydrates are formed earlier. Gas hydrates are composed of mostly the liquid phase (approximately 87 percent). Therefore, it is expected that a higher presence of the liquid phase (water) results in a higher mass transfer rate from the liquid phase to the hydrate phase. 


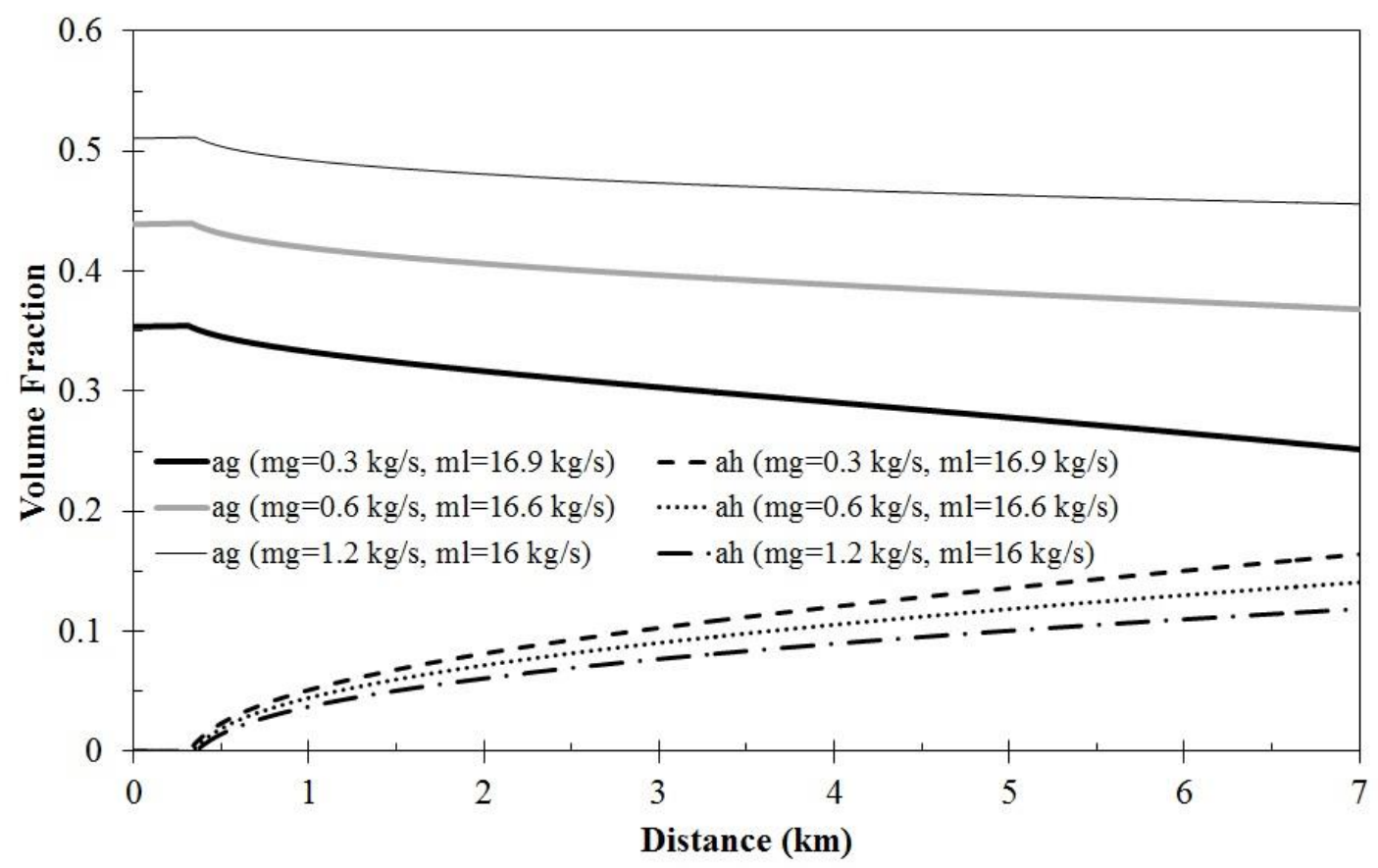

Figure 31. Volume fraction distribution of the gas and hydrate along the pipeline for different mass flow rates

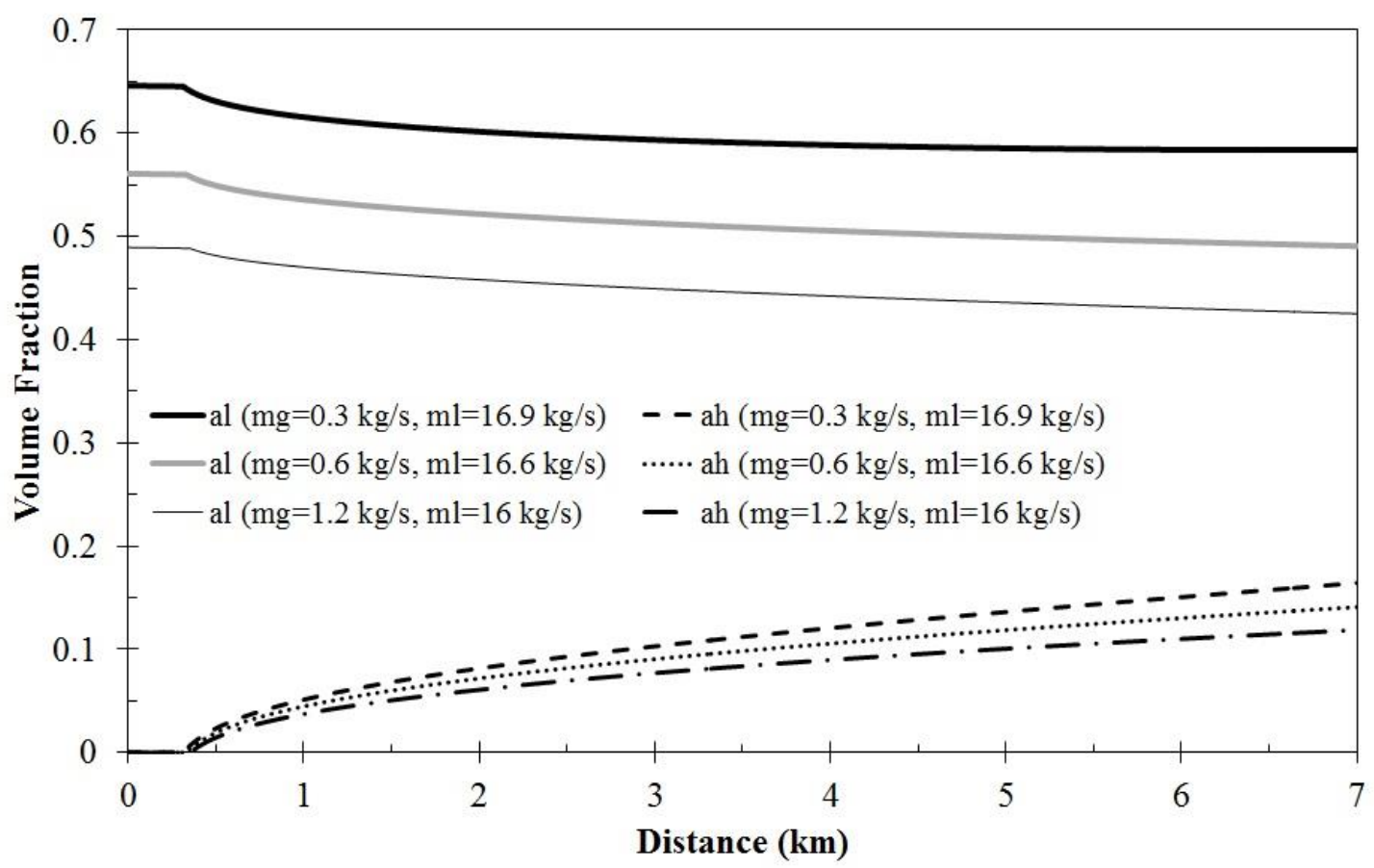

Figure 32. Volume fraction distribution of the liquid and hydrate along the pipeline for different mass flow rates $(\mathrm{al}=$ gas volume fraction, $\mathrm{ah}=$ hydrate volume fraction, $\mathrm{mg}=$ gas flow rate, $\mathrm{ml}=$ liquid flow rate) 
It is expected that the liquid and gas have opposite trends when varying the mass flow rates. This can be verified through Fig. 32, where the distribution of the liquid phase volume fraction along the pipeline is presented. The volume fraction of liquid in the system is directly dependent on the liquid mass flow rate. Therefore, higher mass flow rates for the liquid yields a higher proportion of the pipe volume that is occupied by the liquid phase.

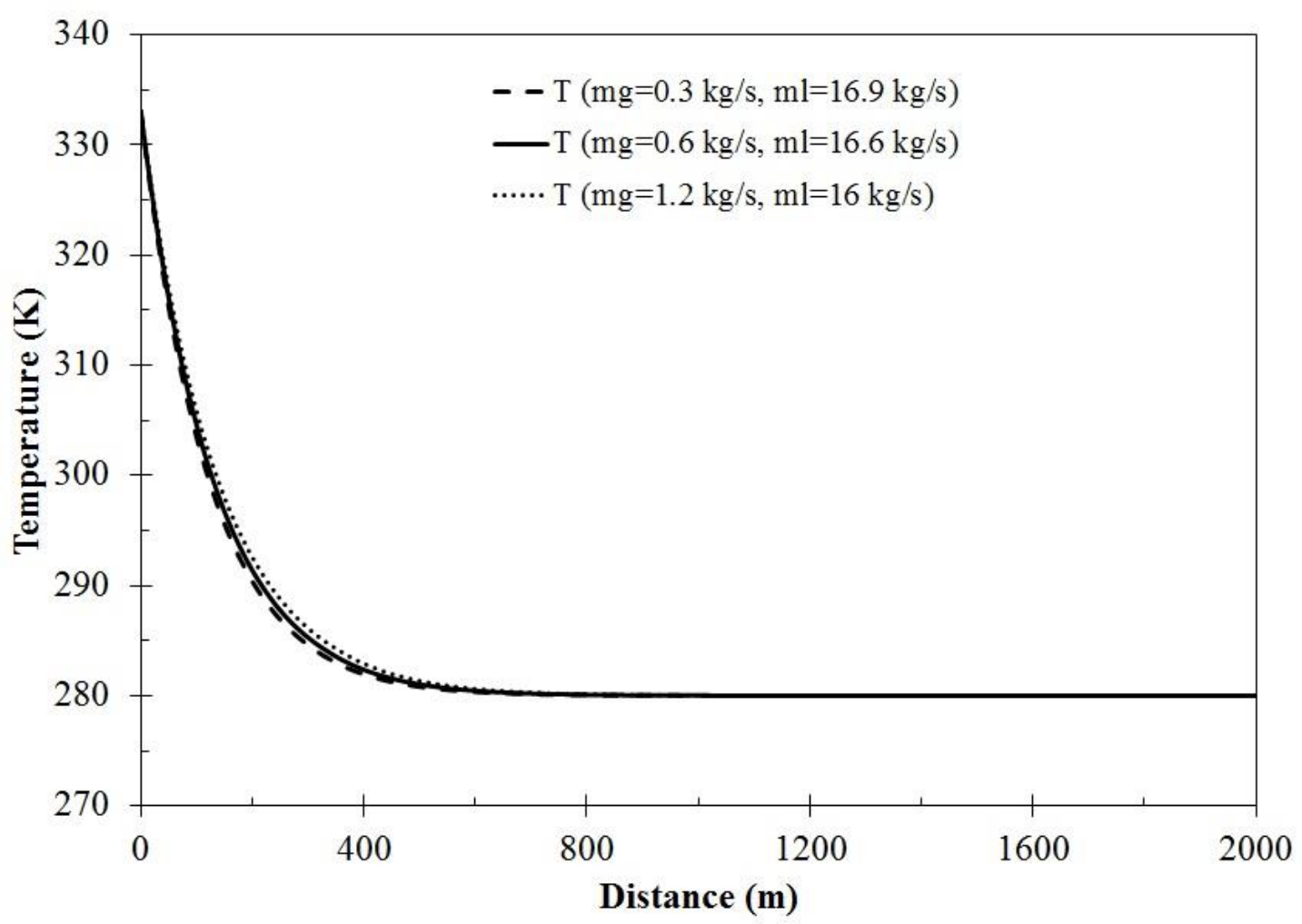

Figure 33. Temperature distribution of the mixture fluid along the pipeline for different mass flow rates

The effect of varying the mass flow rates on the mixture temperature has been shown in Figure 33. Due to the higher conductivity of water compared to gas, the average mixture conductivity increases with a rise in water fraction. This means that the convection coefficient, which is a nearly linear function of the conductivity, increases as well. As a result of a higher convective coefficient, the heat transfer from the system to the surrounding environment is faster, lowering the temperature of the mixture in the system. This is verified 
through Fig. 33, where little difference is seen in the temperature profile due to a variation of the mass flow rates.

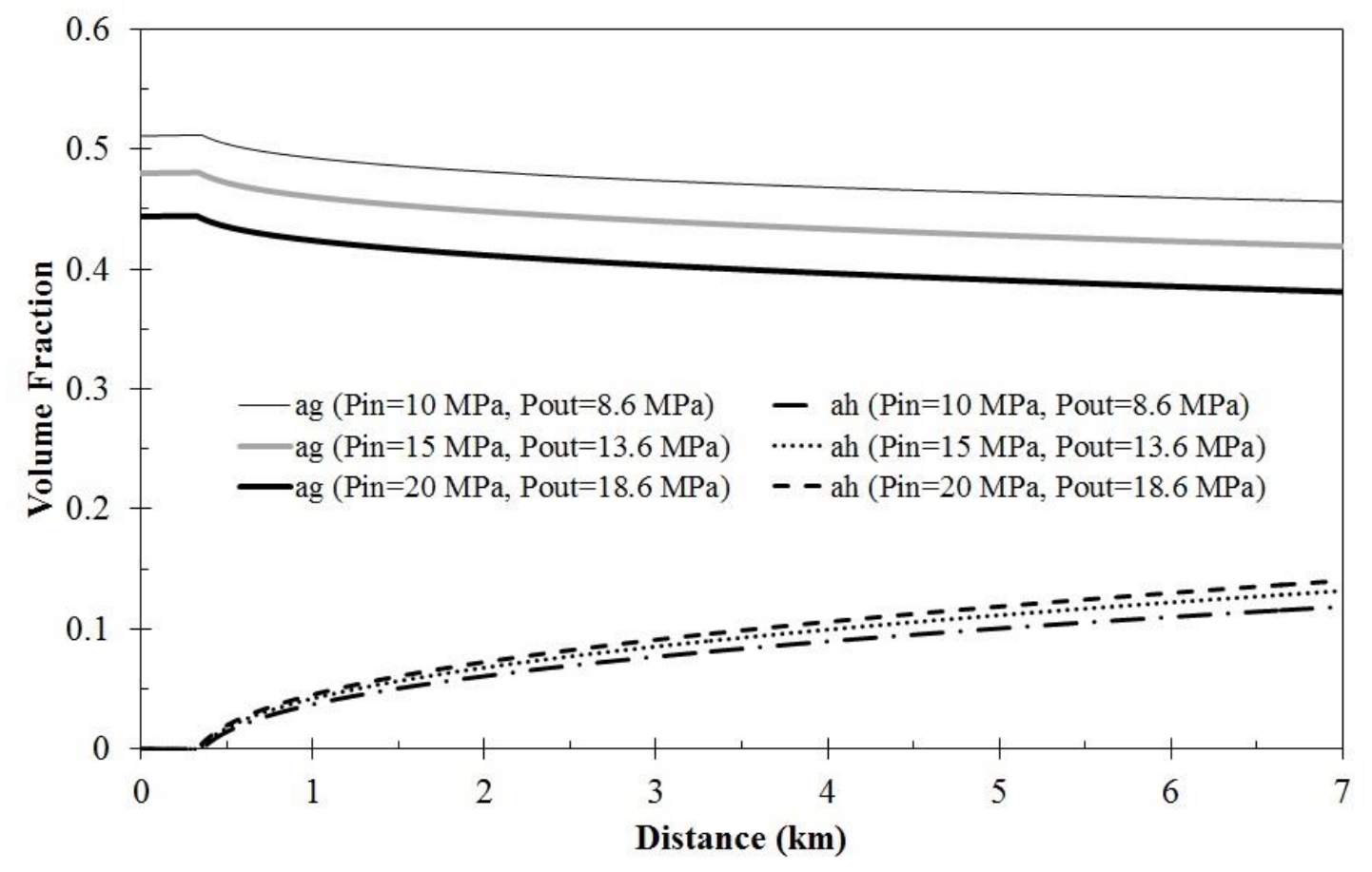

Figure 34. Volume fraction distribution of the gas and hydrate along the pipeline for different

pressures

Figure 34 shows the effect of the change in the system pressure on the volume fraction gas and hydrate. The formation of hydrates depends on the pressure and temperature of the system. Increasing the pressure leads to a higher formation rate of hydrate crystals, meaning a larger volume of the pipe is occupied due to the presence of the hydrates. This means that more gas is taken to form the hydrates. Consequently, the volume fraction of the gas phase has a declining trend along the pipeline. Also, the density of the gas phase is a function of the pressure, and it is higher for larger pressures. Based on Eq. 107, the volume fraction of the gas phase has an inverse relationship with its density. Therefore, it is expected to decrease with an increase in the system pressure (Fig. 34) 


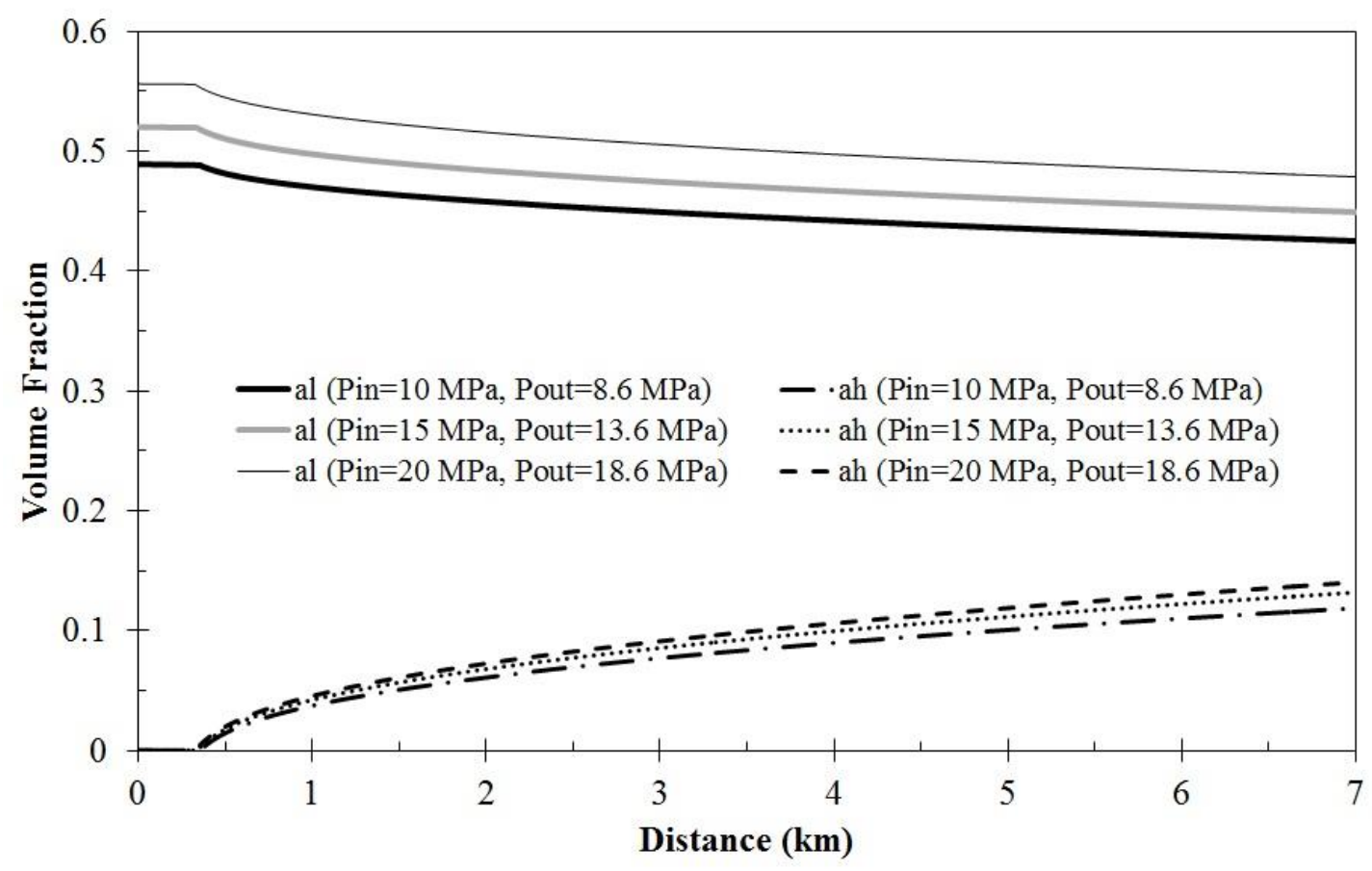

Figure 35. Volume fraction distribution of the liquid and hydrate along the pipeline for different pressures

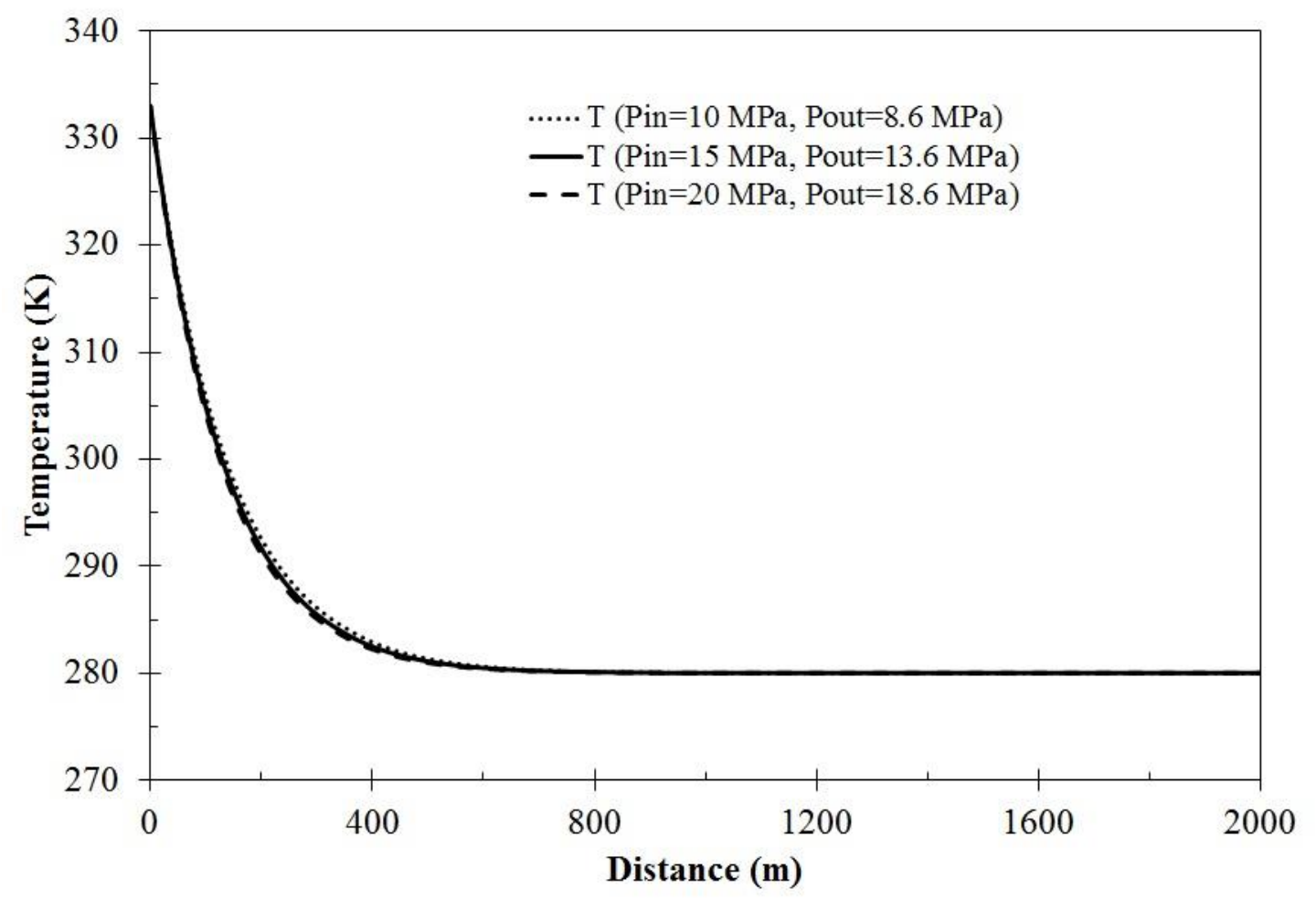

Figure 36. Temperature distribution of the mixture fluid along the pipeline for different pressures The density of the liquid phase is not affected by the pressure. However, the mixture density is related to the liquid velocity. A higher operating pressure reduces the velocity in 
the system, which means the liquid phase volume fraction increases to balance the mass flow rate equation (Eq. 108). Figure 35 verifies this trend and shows the change in the liquid volume fraction along the pipeline for the different pressures.

The temperature profile (Fig. 36) shows that the effect of the variation in pressure is not significant on the temperature distribution along the pipeline. Heat transfer, as a function of the convection coefficient, is not affected by the change in pressure. The increasing trend in the density and the declining trend in the velocity offset each other's effects, causing the Reynolds number to remain almost unchanged. Consequently, the Reynolds number, Nusselt number, and the convection coefficient are not affected, meaning the heat transfer rate is relatively unchanged.

Another factor considered is the effect of inlet temperature on flow characteristics.

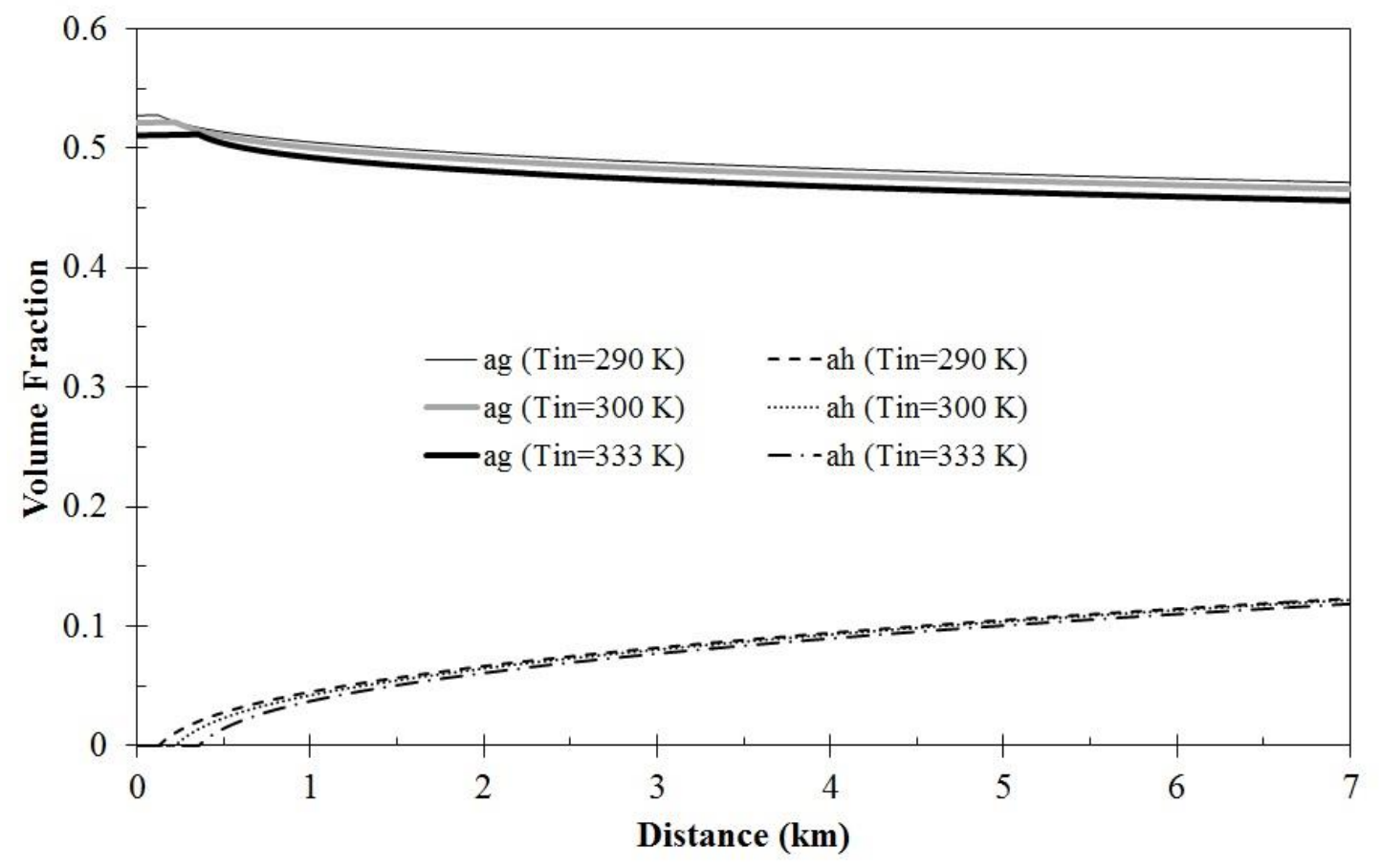

Figure 37. Volume fraction distribution of the gas and hydrate along the pipeline for different inlet temperatures

Figure 37 shows the profile of volume fraction for the gas and hydrate phases along the pipeline for three different inlet temperatures. As expected, for lower inlet temperatures, 
the hydrate particles are formed earlier. Increasing the temperature moves the system condition away from equilibrium conditions and delays the formation stage of hydrate crystals.

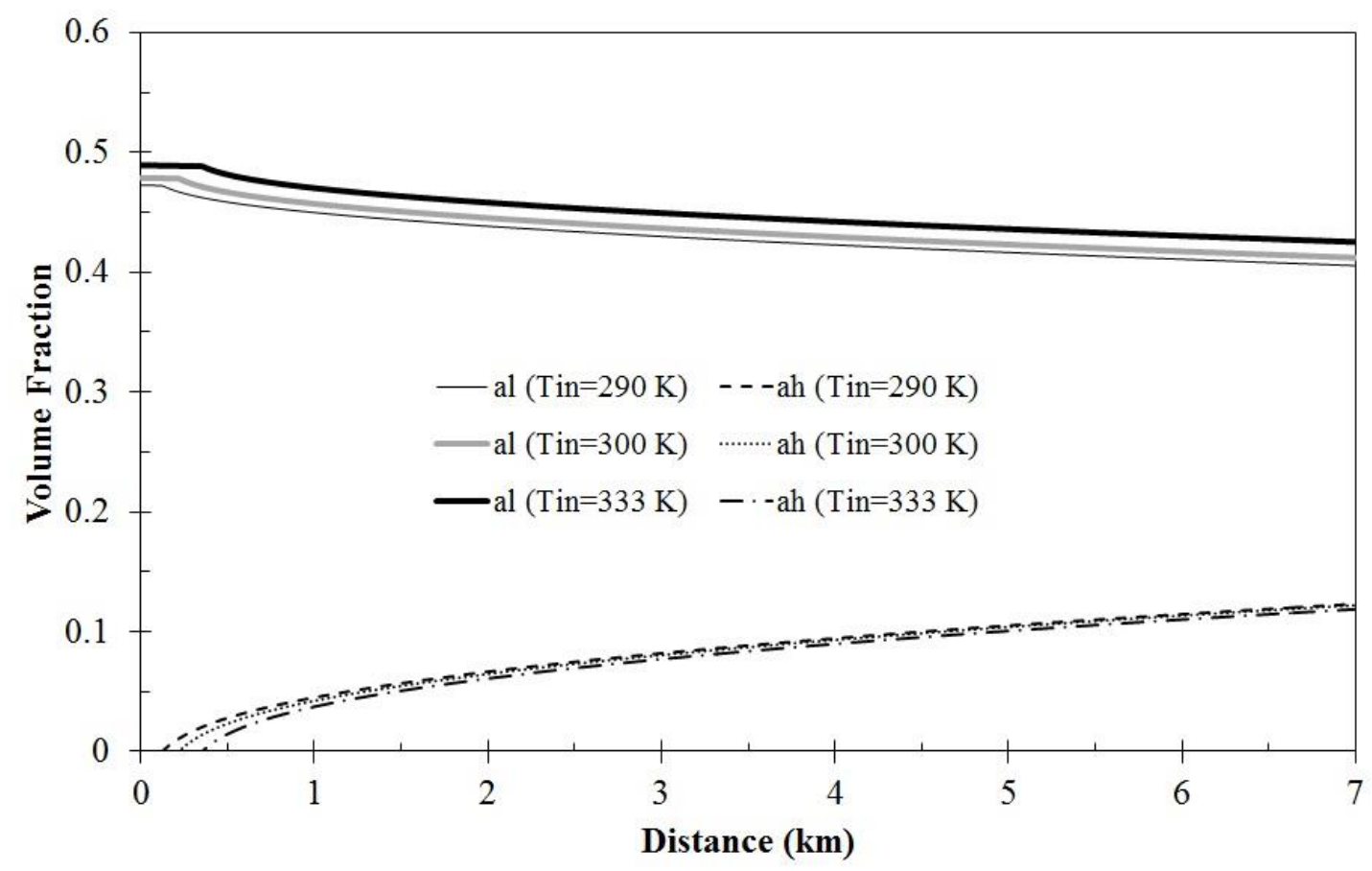

Figure 38. Volume fraction distribution of the liquid and hydrate along the pipeline for different inlet temperatures

Figure 38 illustrates the change in the liquid and hydrate volume fractions along the pipe length. As shown in Figs. 37 and 38, a change of the inlet temperature does not have a significant effect on the volume fraction of the three phases. It, however, can move the location of the initial hydrate formation to an earlier position along the pipeline.

Reducing the inlet temperature decreases the temperature of the entire mixture along the pipeline. Therefore, the temperature meets the equilibrium conditions faster, causing earlier formation of hydrate particles. The reduced temperature mainly affects the region around the pipeline entry. Then, the temperatures drop to the surrounding temperature and remain nearly constant until the pipeline outlet. 


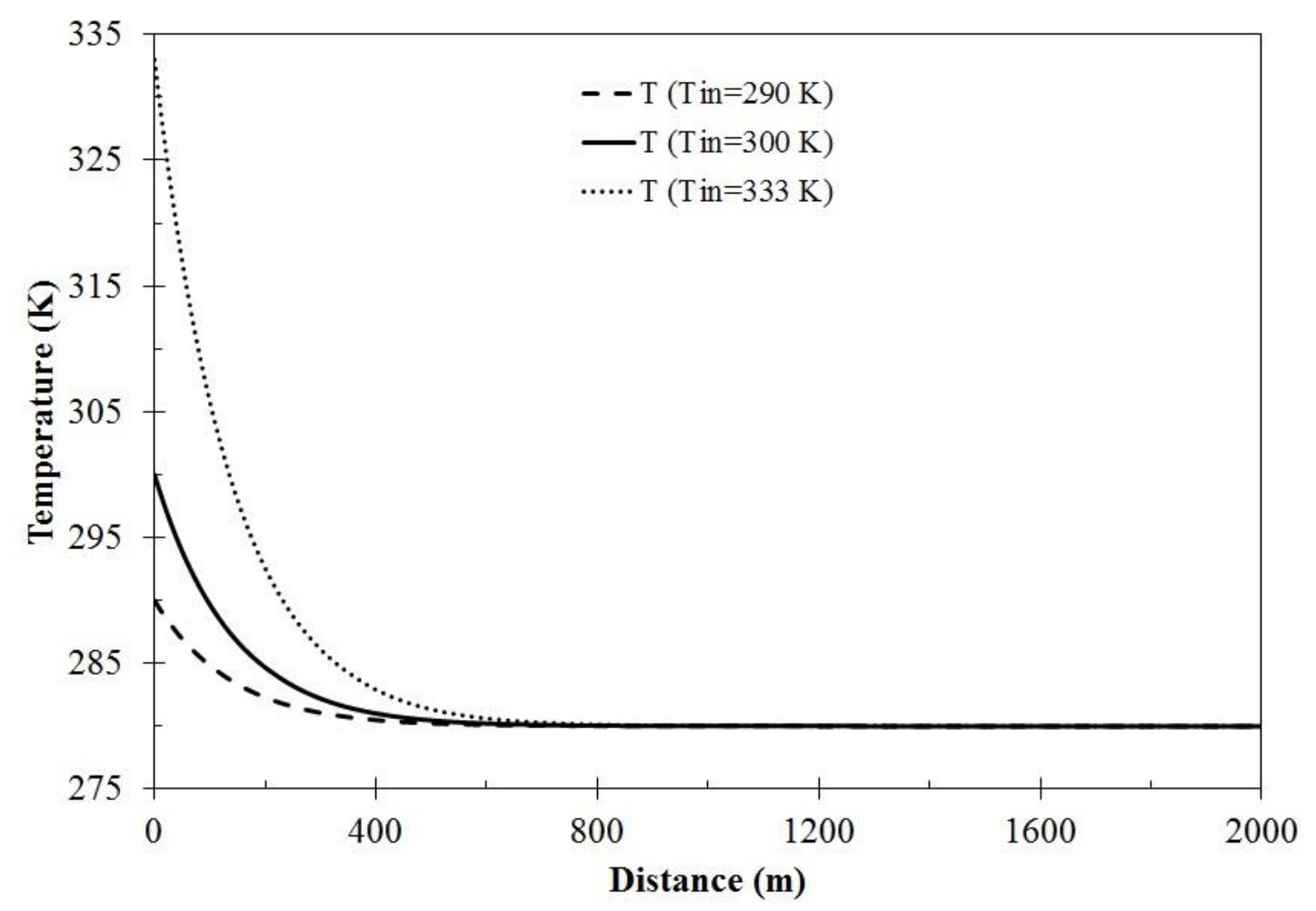

Figure 39. Temperature distribution of the mixture fluid along the pipeline for different inlet temperatures

From the parametric study, it is evident that there is insignificant change to hydrate formation due to pressure and temperature change. This is due to the low hydrate formation rate, which is caused by a sharp decline in the subcooling temperature. The fast drop in the mixture temperature remarkably decreases the subcooling temperature and results in lower rate of hydrate formation. 


\section{CONCLUSIONS AND RECOMMENDATIONS FOR FUTURE RESEARCH}

\subsection{Conclusions}

Two-phase steady-state models for gas/liquid and gas/hydrate flows have been developed to predict two-phase flow in subsea pipelines. Governing equations were solved semi-numerically using MATLAB. The two-phase models have been compared to a singlephase model with mixture properties, and the results including pressure drop and temperature distribution have been found to match well. In the two-phase models, the effect of outlet pressure on the flow characteristics has been investigated. The results show that increases in the outlet pressure reduces the fluid pressure drop and velocity rise, but it does not have a significant effect on temperature distribution of the fluid. The results also reveal that for a given condition, the gas volume fraction increases along the pipeline causing the liquid volume fraction to decrease.

Based on these two-phase models, a three-phase model was also developed to predict the flow of gas, water, and hydrates along a subsea pipeline. Prior to the development of this model, a model for the growth of hydrate particles was also developed. The proposed hydrate growth model was then implemented into the governing equations for the three-phase flow problem. The governing equations were then solved based on an analytical-numerical approach using the Newton-Raphson method. An algorithm was developed in Matlab software to solve the equations from the inlet to outlet of the pipeline.

The results of this study are able to predict the volume fraction and velocity of each phase, as well as the mixture pressure and temperature profiles along the length of the pipeline. The results from the hydrate growth model reveal the growth and the location where the initial hydrates start to form. Finally, to assess the impact of certain parameters on the flow characteristics, parametric studies have been conducted. The results show the effects of variations in the pipe diameter, mass flow rate, inlet pressure, and inlet temperature on the 
flow characteristics and hydrate growth.

\subsection{Recommendations for future research}

Future work should expand the experimental validation of the model that could contribute to a better and more accurate model for the flow in subsea pipelines. Also, a numerical CFD model can be developed to verify the results of this study. Experimental studies could be conducted to evaluate the effects of hydrate growth on flow characteristics. One of the challenging problems for experimental studies is the difficulty of representing full-scale conditions. Achieving a high pressure to form hydrates is not straightforward, limiting the possibilities for observable experimental data.

The proposed three-phase model can also be expanded to a four-phase model by consideration of oil and water as separate fluids. This can lead to a more realistic condition which exists in subsea pipelines. Due to the addition of the oil phase, the interaction between the phases would be more complicated. The modeling would need to consider more equations, as well as more terms in the conservation equations. The hydrate phase may also form at the gas/oil interface and enter the oil phase.

Finally, a transient analysis can be conducted to study the four-phase transient flow characteristics along the pipeline for different time scales. The study can also examine the hydrate formation and growth over time to determine when and where the hydrates are formed. 


\section{REFERENCES}

Aakenes, F., (2012), "Frictional pressure drop models for steady-state and transient twophase flow of carbon dioxide", M.Sc. thesis, Norwegian University of Science and Technology

Abbaspour, M., Champan, K. S., and Glasgow, L. A., (2010), “Transient modeling of nonisothermal, dispersed two-phase flow in natural gas pipelines”, Applied Mathematical Modeling, 34, pp. 495-507

Acikgoz, M., Franca, F., and Lahey, R. T., (1992), “An experimental study of three-phase flow regimes", International Journal of Multiphase Flow, 18 (3), 327-336

Balasubramaniam, R., Ramé E., Kizito J., and Kassemi M., (2006), "Two phase flow modeling summary of flow regimes and pressure drop correlations in reduced and partial gravity", National Aeronautics and Space Administration, Glenn Research Center, Cleveland, Ohio

Bar-Meir, G., "Basics of fluid mechanics", (2013), Last modified: version 0.3.4.0, www.potto.org/downloads.php

Bendiksen, K., (2012), "Multiphase flow modelling and its significance for subsea field developments on the NCS", The Tekna Conference on MP Flow Systems, Oslo, Norway

Bendiksen, K. H., Malnes, D., Moe, R., and Nuland, S., (1991), "The dynamic two-fluid model OLGA: theory and application", Society of Petroleum Engineers, pp. 171-180

Bergman, T. L., Lavine, A. S., Incropera, F. P., and Dewitt, D. P., (2011), "Fundamentals of heat and mass transfer", $7^{\text {th }}$ edition, John Wiley \& Sons

Bonizzi, M. and Issa, R. I., (2003), “On the simulation of three-phase slug flow in nearly horizontal pipes using the multi-fluid model", International Journal of Multiphase Flow, 29, pp. 1719-1747 
Bratland, O., (2009), "Pipe flow 1, single-phase flow assurance", http://www.drbratland.com/ Bratland, O., (2010), "Pipe flow 2, multi-phase flow assurance”, http://www.drbratland.com/ Brennen, C. E., (2005), “Fundamentals of multiphase flows”, Cambridge University Press Brill, J.P. and Mukherjee, H., (1999), "Multiphase flow in wells", SPE Monograph Series, Society of Petroleum Engineers, Richardson, Texas

Cheng, N., (2009), "Comparison of formulas for drag coefficient and settling velocity of spherical particles”, Powder Technology, 189 (3), pp. 395-398

Creek, J.L., Subramanian, S., and Estanga, D., (2011), "New method for managing hydrates in deepwater tiebacks", Offshore Technology Conference, Doi: 10.4043/22017-MS, Houston, Texas, USA

Danielson, T. J., (2012), "Transient multiphase flow: past, present, and future with flow assurance perspective", Energy \& Fuels, 26 (7), pp. 4137-4144, doi: $10.1021 / \mathrm{ef} 300300 \mathrm{u}$

Danielson, T.J., (2011), “A simple model for hydrodynamic slug flow”, Offshore Technology Conference, Houston, Texas, USA, Doi: 10.4043/21255-MS

De Henau, V. and Raithby, G. D., (1995), "A transient two-fluid model for the simulation of slug flow in pipelines-I. Theory”, International Journal of Multiphase Flow, 21, pp. $335-349$

Fang, X., Xu, Y., and Zhou, Z., (2011), "New correlations of single-phase friction factor for turbulent pipe flow and evaluation of existing single-phase friction factor correlations", Nuclear Engineering and Design, 241, pp. 897-902

Gregory, G. A. and Forgarasi, M., (1985), “A critical evaluation of multiphase gas/liquid pipeline calculation methods", $2^{\text {nd }}$ International Conference on Multiphase Flow, London, 121, pp. 93-108

Hammerschmidt, E.G., (1934), "Formation of gas hydrates in natural gas transmission lines, 
Industrial \& Engineering Chemistry, 26 (8), pp. 851-855

Hanratty, T. J., (2003), "Workshop on scientific issues in multiphase flow", Department of Chemical and Biomolecular Engineering at University of Illinois, USA

Joshi, S. V., Grasso, G. A., Lafond, P. G., Rao, I., Webb, E., Zerpa, L. E., Sloan, E. D., Koh, C. A., and Sum, A. K., (2013), "Experimental flowloop investigations of gas hydrate formation in high water cut systems", Chemical Engineering Science, 97, pp. 198209, doi: 10.1016/j.ces.2013.04.019

Joshi, S., Zerpa, L. E., Aman, Z. M., Rao, I., Sloan, E. D., Koh, C., and Sum, A., (2012), "Predicting hydrate blockages in oil, gas and water-dominated systems", Offshore Technology Conference, doi:10.4043/23490-MS, Houston, Texas, USA

Jung, J. W. and Santamarina, J. C., (2012), "Hydrate formation and growth in pores", Journal of Crystal Growth, 345, pp. 61-68

Kang, Y., Kwang, J., Ko, M., Cho, Y., and Kim, S., (1999), "Particle flow behavior in threephase fluidized beds”, Korean Journal of Chemical Engineering, 16, pp. 784-788

Khor, S. H., Mendes-Tatsis, M. A., and Hewitt, G. F., (1997), "One-dimensional modeling of phase holdups in three-phase stratified flow", International Journal of Multiphase Flow, 23 (5), pp. 885-897

Kurup, A. S., Vargas, F. M., Wang, J. X., Buckley, J., Creek, J. L., Subramani, H. J., and Chapman, W. G., (2011), “Development and application of an asphaltene deposition tool (adept) for well bores", Energy \& Fuels, 25 (10), pp. 4506-4516. doi: $10.1021 /$ ef200785v

Kvamme, B., (1996), “A new theory for the kinetics of hydrate formation”, $2^{\text {nd }}$ International Conference on Natural Gas Hydrates, Toulouse, France

Kwon, O., (1999), "Numerical modeling study for the analysis of multiphase transient flow of natural gas/oil/water/hydrate through seabed pipeline", $\mathrm{PhD}$ dissertation, Hanyang 
university, Seoul, Korea

Kwon, O., Ryou, S., and Sung, W., (2001), "Numerical modeling study for the analysis of transient flow characteristics of gas, oil, water, and hydrate flow through a pipeline", Korean Journal of Chemical Engineering, 18 (1), pp. 88-93

Lachance, J. W., Talley, L. D., Shatto, D. P., Turner, D. J., and Eaton, M. W., (2012), "Formation of hydrate slurries in a once-through operation", Energy \& Fuels, 26 (7), pp. 4059-4066, doi: 10.1021/e13002197

Lockhart, R.W. and R.C. Martinelli, (1949), "Proposed correlation of data for isothermal two-phase, two-component flow in pipes”, Chemical Engineering Progress, 45 (1), pp. $39-48$

Mackintosh, N. and Atakan. Z., (2000), "Flow assurance still leading concern among producers", Offshore, $60(10)$

Mork, M. and Gudmundsson, J.S., (2001), "Rate of hydrate formation in subsea pipelines, correlation based on reactor experiments", $12^{\text {th }}$ International Oil Filed Chemistry Symposium, Geilo, Norway

Nicholas, J. W., Inman, R. R., Steele, J. P. H., Koh, C. A., and Sloan, E. D., (2008), “A modeling approach to hydrate wall growth and sloughing in a water saturated gas pipeline", 6th International Conference on Gas Hydrates, Vancouver, British Columbia, Canada

Nicholas, J. W., Koh, C. A., Sloan, E. D., Nuebling, L., He, H., and Horn, B, (2009), "Measuring hydrate/ice deposition in a flow loop from dissolved water in live liquid condensate", Aiche Journal, 55 (7), pp. 1882-1888, doi: 10.1002/aic.11874

Ouyang, L. and Aziz, K., (2001), “Transient gas-liquid two-phase flow in pipes with radial influx or efflux", Journal of Petroleum Science and Engineering, 30, pp. 167-179 
Petalas, N. and Aziz, K., (1997), “A Mechanistic Model for Stabilized Multiphase Flow in Pipes”, Report for the Stanford Reservoir Simulation Industrial Affiliates and the Stanford Project on the Productivity and Injectivity of Horizontal Wells, Petroleum Engineering Department, Stanford University, Stanford, CA, USA

Saito, T., Hughes, E. D., and Carbon, M. W., (1978), "Multi-fluid modeling of annular twophase flow", Nuclear Engineering and Design, 50, pp. 225-271

Singh, P., Venkatesan, R., Fogler, H. S., and Nagarajan, N., (2000), "Formation and aging of incipient thin film wax-oil gels", Aiche Journal, 46 (5), pp. 1059-1074, doi: 10.1002/aic.690460517

Sloan, E. D., Koh, C. A., and Sum, A. K. (2011), "Natural gas hydrates in flow assurance", Burlington, Gulf Professional Publication, Boston, pp. 1-11

Taitel, Y, Barnea, D., and Brill, J. P., (1995), "Stratified three-phase flow in pipes", International Journal of Multiphase Flow, 21 (1), pp. 53-60

Taitel, Y. and Dukler, A. E., (1976), "A model for predicting flow regime transitions in horizontal and near horizontal gas/liquid flow", American Institute of Chemical Engineers Journal, 22 (1), pp. 47-55

Tek, R., (1961), "Multiphase flow of water, oil and natural gas through vertical flow strings", Journal of Petroleum Technology, 13 (10), pp. 1029-1936

Thome, J. R., (2006), “Engineering data book III”, Wolverine Tube, Inc.

Tso, C. P. and Sugawara, S., (1990), "Film thickness prediction in a horizontal annular twophase flow", International Journal of Multiphase Flow, 16 (5), pp. 867-884

Turner, D. J., Boxall, J., Yang, S., Kleehammer, D. M., Koh, C. A., Miller, K. T., and Sloan, E. D., (2005), "Development of a hydrate kinetic model and its incorporation into the OLGA2000 transient multi-phase flow simulator", 5th International Conference on Gas Hydrates, 4018, pp. 1231-1240, Trondheim, Norway 
Urroz, G. E., (2004), “Solution of non-linear equations”, pp. 1-18

Vysniauskas, A. and Bishnoi, P. R., (1983), “A kinetic-study of methane hydrate formation”, Chemical Engineering Science, 38 (7), doi: 10.1016/0009-2509(83)80027-x

Whitaker S., (1972), "Forced convection heat transfer correlation for flow in pipes, past flat plates, single cylinders, single spheres and flow in packed beds and tube bundles", AICHE Journal, 18, pp. 361-371

White, F. M., (2008), "Fluid Mechanics", 6th edition, McGraw-Hill book Companies, New York

Wylie, E. B., Streeter, V. L., and Stoner, M. A., (1974), "Unsteady-state natural gas calculations in complex pipe systems", Society of Petroleum Engineers, pp. 35-43

Xiao, J. J., Shoup, G., Hatton, G., and Kruka, V., (1998), "Predicting hydrate plug movement during subsea flowline depressurization operations", Offshore Technology Conference, doi: 10.4043/8728-MS

Yuen, M. C. and Chen, L. W., (1976), “On drag of evaporating liquid droplet”, Combustion Science and Technology, 14, pp. 147-154 